Preprint typeset in JHEP style - HYPER VERSION

DAMTP-2008-16

\title{
General Analysis of LARGE Volume Scenarios with String Loop Moduli Stabilisation
}

\author{
Michele Cicoli, Joseph P. Conlon and Fernando Quevedo \\ DAMTP, Centre for Mathematical Sciences, \\ Wilberforce Road, Cambridge, CB3 0WA, UK. \\ Email: M.Cicoli@damtp.cam.ac.uk, J.P.Conlon@damtp.cam.ac.uk, \\ F.Quevedo@damtp.cam.ac.uk
}

\begin{abstract}
We study the topological conditions for general Calabi-Yaus to get a nonsupersymmetric AdS exponentially large volume minimum of the scalar potential in flux compactifications of IIB string theory. We show that negative Euler number and the existence of at least one blow-up mode resolving point-like singularities are necessary and sufficient conditions for moduli stabilisation with exponentially large volumes. We also analyse the general effects of string loop corrections on this scenario. While the combination of $\alpha^{\prime}$ and nonperturbative corrections are sufficient to stabilise blow-up modes and the overall volume, quantum corrections are needed to stabilise other directions transverse to the overall volume. This allows exponentially large volume minima to be realised for fibration Calabi-Yaus, with the various moduli of the fibration all being stabilised at exponentially large values. String loop corrections may also play a role in stabilising 4-cycles which support chiral matter and cannot enter directly into the non-perturbative superpotential. We illustrate these ideas by studying the scalar potential for various Calabi-Yau three-folds including $K 3$ fibrations and briefly discuss the potential phenomenological and cosmological implications of our results.
\end{abstract}

KEYWORDS: String compactifications, moduli stabilisation. 


\section{Contents}

1. Introduction 1

2. The Large Volume Scenario 3

2.1 Low Energy Limit for Type IIB Flux Compactifications

2.2 General Analysis for the Large Volume Limit 5

3. Particular Examples 7

3.1 The single-hole Swiss cheese: $\mathbb{C} P_{[1,1,1,6,9]}^{4}$

3.2 The multiple-hole Swiss cheese: $\mathcal{F}_{11}$ and $\mathbb{C} P_{[1,3,3,3,5]}^{4}$

3.3 2-Parameter K3 Fibration: $\mathbb{C} P_{[1,1,2,2,6]}^{4}$

3.3.1 Explicit Calculation 10

3.4 3-Parameter K3 Fibration 11

3.4.1 Explicit Calculation

4. Inclusion of the String Loop Corrections 16

4.1 LARGE Volume and String Loop Corrections 18

5. Moduli Stabilisation via String Loop Corrections 19

5.1 The single-hole Swiss cheese: $\mathbb{C} P_{[1,1,1,6,9]}^{4}$

5.2 The multiple-hole Swiss cheese: $\mathbb{C} P_{[1,3,3,3,5]}^{4}$

5.3 2-Parameter K3 Fibration: $\mathbb{C} P_{[1,1,2,2,6]}^{4}$

5.4 3-Parameter K3 Fibration 24

6. Conclusions and Potential Applications 27

A. Proof of the LARGE Volume Claim 29

A.1 Proof for $N_{\text {small }}=1 \quad 29$

A.2 Proof for $N_{\text {small }}>1$

A.2.1 Axion stabilisation 37

A.2.2 Kähler moduli stabilisation 44

A.3 General Picture 61

\section{Introduction}

In constructing string models that can be compared to experiment, it is essential to understand the vacuum state. It is the vacuum state whose properties will give rise to, for example, low-energy supersymmetry and the pattern of Yukawa couplings. For a long time string compactifications were plagued by the problem of moduli. These were massless scalars that parameterised the vacuum and whose VEVs entered into the value of physical observables. The existence of moduli leads to fifth forces and also makes the vacuum inherently unpredictive. Perturbative and nonperturbative quantum effects, generically present 
in $N=1$ supersymmetric theories, were expected to lead to moduli stabilisation but for a long time no controlled procedure existed.

This situation improved remarkably after the systematic study of flux compactifications and their impact on moduli stabilisation [1] (for reviews see [2, 3]) Fluxes are particularly powerful in type IIB string compactifications, as there the backreaction is well-controlled and gives rise only to warped Calabi-Yau metrics. Furthermore, the combination of nonperturbative effects [4, 5] with perturbative $\alpha^{\prime}$ corrections [6, 7, 8 has allowed a general study of the large volume scalar potential for arbitrary Calabi-Yau compactifications and has led to the discovery of exponentially large volume compactifications [8, 9] with very interesting phenomenological and cosmological implications.

If $\alpha^{\prime}$ corrections can play a significant role in moduli stabilisation in the phenomenologically relevant regime of large volume and weak coupling, it is natural to wonder whether $g_{s}$ corrections may also have a significant effect. At first sight this seems unavoidable, as at large volume the corrections to the Kähler potential induced by string loops are parametrically larger than those induced by $\alpha^{\prime}$ corrections [10]. However, the scalar potential exhibits an extended no-scale structure, and the loop corrections contribute to the scalar potential at a level subleading to their contribution to the Kähler potential and subleading to the $\alpha^{\prime}$ corrections [10, 11, 12]. 13] studied the effect of loop corrections on the $\mathbb{C} P_{[1,1,1,6,9]}^{4}$ large volume model and found it only gave minor corrections to the moduli stabilisation and sub-sub-leading corrections to the soft term computation. It is then natural to ask whether loop corrections to the scalar potential can give a qualitative, rather than only quantitative, change to moduli stabilisation.

We study this question in this paper and find that the answer is affirmative. The Large Volume Scenario (LVS) stabilises the overall volume at an exponentially large value using $\alpha^{\prime}$ and $g_{s}$ corrections. Most previous work has focused on 'Swiss-Cheese' Calabi-Yaus, where one cycle controls the overall volume ('size of the cheese') and the remaining moduli control the volume of blow-up cycles ('holes in the cheese'). However for Calabi-Yaus with a fibration structure - the torus is the simplest example - multiple moduli enter into the overall volume. For the overall volume to be made large in a homogeneous fashion, several moduli must become large. In these cases, while the existence of at least one blow-up mode is still necessary, loop corrections turn out to be necessary in order to realise the LVS and obtain a stable minimum at exponentially large volume. The loop corrections lift directions transverse to the overall breathing mode and stabilise these.

The fact that the volume is stabilised large is desirable, first to trust the perturbative expansion in the low energy effective field theory and secondly as a powerful tool to generate hierarchies. Moreover we stress that this minimum is found for generic values of $W_{0}$, without the need to fine tune $W_{0}$.

We will illustrate our results by applying them to some examples of Calabi-Yau threefolds that are hypersurfaces in complex weighted projected spaces. From this analysis, it turns out that a necessary and sufficient condition for LARGE volume is the presence of blow-up modes resolving point-like singularities. It seems also that the Calabi-Yaus which have a fibration structure cannot present the interesting phenomenological properties of the LVS. However, as we will explain in Sections 1 and 5 , via the inclusion of the string loop 
corrections to the Kähler potential, those K3 fibrations can also present an exponentially large volume minimum, provided a blow-up mode exists.

We will also show that the string loop corrections may play a role in addressing the problem stressed in 14. The authors there argued that the 4-cycle on which the Standard Model lives cannot get non-perturbative corrections since their prefactor is proportional to the VEV of Standard Model fields which, at this stage, is required to vanish. However, due to the constraints of the Standard Model gauge couplings this cycle must still be stabilised at a relatively small size.

This problem may be cured through having at least two blow-up modes and then adding $g_{s}$ corrections. The loop corrections have the ability to stabilise the Standard Model cycle, while the 'transverse' cycle is stabilised non-perturbatively as usual. This possible solution is discussed for the example $\mathbb{C} P_{[1,3,3,3,5]}^{4}$, studied in detail in Section 5.2 . We will see that the inclusion of the $g_{s}$ corrections can freeze $\tau_{S M}$ small producing a minimum of the full scalar potential at exponentially large volume.

This paper is organised as follows. In the next section we briefly review the LARGE Volume Scenario and state the general conditions that have to be satisfied in order to have exponentially large volume. The detailed proof of this general result is left to the appendix. Section 3 illustrates our general results for several Calabi-Yaus, including both Swiss cheese models where all Kähler moduli other than the overall volume are blowups, and also fibration Calabi-Yaus, such as K3 fibrations. Section 4 reviews the general structure of string loop corrections and section 5 illustrates how these corrections affect the results on the models of section 3 . We finish with a short section on conclusions and potential applications of our results.

We shall not discuss obtaining be Sitter vacua in this paper. For a recent analysis of the conditions for de Sitter vacua from supergravity, see [15].

\section{The Large Volume Scenario}

Large volume compactifications of string theories are desirable for several reasons. First, large volume allows massive string states to be consistently integrated out and makes the effective field theory description of the compactification more robust. Second, large

volume is desirable for phenomenological reasons. Given the standard relation $M_{\text {planck }}^{2} \sim$ $\frac{M_{\text {string }}^{2} \mathcal{V}}{g_{s}}$, it is clear that LARGE volume (or WEAK coupling [16]) is necessary to have the fundamental string scale hierarchically smaller than the Planck scale. In string units a volume $\mathcal{V} \sim 10^{4}$ is needed for $M_{\text {string }}=M_{G U T}$ and much larger volumes are needed for an intermediate scale $M_{\text {string }} \sim 10^{11} \mathrm{GeV}\left(\mathcal{V} \sim 10^{15}\right)$, as attractive for gravity mediated supersymmetry breaking, or $M_{\text {string }} \sim 1 \mathrm{TeV}\left(\mathcal{V} \sim 10^{30}\right)$ in the extreme case of $\mathrm{TeV}$ strings.

Explicitly obtaining exponentially large volume in string theory, with all the geometric moduli stabilised, goes much farther than the original large extra dimensions proposals 17] where the volume was simply assumed to be large. In this section we will briefly review how moduli are typically stabilised in IIB string theory and then we will describe the 
general conditions under which an exponentially large volume Calabi-Yau compactification is obtained.

\subsection{Low Energy Limit for Type IIB Flux Compactifications}

To establish notation and conventions we start with a rapid review of type IIB flux compactifications on Calabi-Yau three-folds $X$ [1], dimensionally reduced to $4 \mathrm{D} \mathcal{N}=1$ supergravity. The Kähler potential takes the form:

$$
K=-2 \ln \left(\mathcal{V}+\frac{\xi}{2 g_{s}^{3 / 2}}\right)-\ln (S+\bar{S})-\ln \left(-i \int_{X} \Omega \wedge \bar{\Omega}\right) .
$$

In (2.1) we have included the leading $\alpha^{\prime}$ correction but have not included any string loop corrections. The constant $\xi$ is given by: $\xi=-\frac{\chi(X) \zeta(3)}{2(2 \pi)^{3}}$, where $\chi$ is the Euler number of the Calabi-Yau $X, \zeta(3) \approx 1.2, S$ is the axio-dilaton, $S=e^{-\varphi}+i C_{0}$, and $\Omega$ is the holomorphic $(3,0)$-form which implicitly depends on the complex structure moduli $U_{\alpha}, \alpha=1, \ldots, h_{2,1}(X)$. $\mathcal{V}$ is the internal volume, measured with an Einstein frame metric $g_{\mu \nu, E}=e^{-\varphi / 2} g_{\mu \nu, s}$ and in units of $l_{s}=2 \pi \sqrt{\alpha^{\prime}}$. It can be expressed in terms of the Kähler form $J$ once this is expanded in a base $\left\{\hat{D}_{i}\right\}$ of $H^{1,1}\left(X, \mathbb{Z}\right.$ ) as $J=\sum_{i=1}^{h_{1,1}} t^{i} \hat{D}_{i}$ (we focus on orientifold projections such that $\left.h_{1,1}^{-}=0 \Rightarrow h_{1,1}^{+}=h_{1,1}\right)$ :

$$
\mathcal{V}=\frac{1}{6} \int_{X} J \wedge J \wedge J=\frac{1}{6} k_{i j k} t^{i} t^{j} t^{k}
$$

Here $k_{i j k}$ are the triple intersection numbers of $X$ and the $t^{i}$ are 2-cycle volumes.

The fields entering the $\mathcal{N}=1$ chiral multiplets are the complexified Kähler moduli $T_{i}=\tau_{i}+i b_{i}$ where $\tau_{i}$ is the Einstein frame volume (in units of $l_{s}$ ) of the divisor $D_{i} \in$ $H_{4}(X, \mathbb{Z})$, which is the Poincaré dual to $\hat{D}_{i}$. Its axionic partner $b_{i}$ is the component of the RR 4-form $C_{4}$ along this cycle: $\int_{D_{i}} C_{4}=b_{i}$. The 4-cycle volumes $\tau_{i}$ are related to the 2-cycle volumes $t^{i}$ :

$$
\tau_{i}=\frac{\partial \mathcal{V}}{\partial t^{i}}=\frac{1}{2} \int_{X} \hat{D}_{i} \wedge J \wedge J=\frac{1}{2} k_{i j k} t^{j} t^{k} .
$$

The classical superpotential is generated by background fluxes $G_{3}=F_{3}+i \mathrm{SH}_{3}$, where $F_{3}$ and $H_{3}$ are the RR and NSNS 3-form fluxes respectively. This superpotential also receives nonperturbative corrections from either brane instantons $\left(a_{i}=2 \pi\right)$ or gaugino condensation $\left(a_{i}=2 \pi / N\right)$. The full superpotential is then:

$$
W=W_{\text {tree }}+W_{n p}=\int_{X} G_{3} \wedge \Omega+\sum_{i} A_{i} e^{-a_{i} T_{i}} .
$$

The sum is over cycles generating nonperturbative contributions to $W$. The $A_{i}$ correspond to threshold effects and depend on the complex structure moduli and positions of D3branes. There may additionally be higher instanton effects in (2.4), but these can be neglected so long as each $\tau_{i}$ is stabilised such that $a_{i} \tau_{i} \gg 1$. 
The $\mathcal{N}=1 \mathrm{~F}$-term supergravity scalar potential is given by:

$$
V=e^{K}\left\{\sum_{i=T, S, U} K_{i \bar{j}}^{-1} D_{i} W D_{\bar{j}} \bar{W}-3|W|^{2}\right\},
$$

where

$$
\left\{\begin{array}{l}
D_{i} W=\partial_{i} W+W \partial_{i} K, \\
D_{\bar{j}} \bar{W}=\partial_{\bar{j}} \bar{W}+\bar{W} \partial_{\bar{j}} K .
\end{array}\right.
$$

Classically, the potential for the Kähler moduli is flat due to the no-scale structure:

$$
\left(\frac{\partial^{2} K_{\text {tree }}}{\partial T_{i} \partial \bar{T}_{j}}\right)^{-1} \frac{\partial K_{\text {tree }}}{\partial T_{i}} \frac{\partial K_{\text {tree }}}{\partial \bar{T}_{j}}=3 .
$$

This implies that the dilaton and complex structure moduli are stabilised supersymmetrically at tree level, $D_{S} W=D_{U} W=0$.

The classical flatness of the potential for the Kähler moduli implies that to study Kähler moduli stabilisation we should keep all possible quantum corrections, while for the $U$ and $S$ moduli it is sufficient to stabilise them classically:

$$
\left\{\begin{array}{l}
K=K_{c s}-2 \ln \left(\frac{2}{g_{s}}\right)-2 \ln \left(\mathcal{V}+\frac{\xi}{2 g_{s}^{3 / 2}}\right), \\
W=W_{0}+\sum_{i} A_{i} e^{-a_{i} T_{i}}
\end{array}\right.
$$

where $K_{c s}=\left\langle-\ln \left(-i \int_{X} \Omega \wedge \bar{\Omega}\right)\right\rangle$ and $W_{0}=\left\langle W_{\text {tree }}\right\rangle$. Substituting (2.8) in (2.5), we obtain the following potential (defining $\hat{\xi} \equiv \xi / g_{s}^{3 / 2}$ ):

$$
\begin{aligned}
V= & e^{K}\left[K_{0}^{j k}\left(a_{j} A_{j} a_{k} \bar{A}_{k} e^{-\left(a_{j} T_{j}+a_{k} \bar{T}_{k}\right)}-\left(a_{j} A_{j} e^{-a_{j} T_{j}} \bar{W} \partial_{\bar{T}_{k}} K_{0}+a_{k} \bar{A}_{k} e^{-a_{k} \bar{T}_{k}} W \partial_{T_{j}} K_{0}\right)\right)\right. \\
& \left.+3 \hat{\xi} \frac{\left(\hat{\xi}^{2}+7 \hat{\xi} \mathcal{V}+\mathcal{V}^{2}\right)}{(\mathcal{V}-\hat{\xi})(2 \mathcal{V}+\hat{\xi})^{2}}|W|^{2}\right] .
\end{aligned}
$$

The aim of this paper will be a detailed study of the potential (2.9) for various different Calabi-Yaus, incorporating string loop corrections into the form of the Kähler potential. We aim to work out conditions for when the exponentially large volume minimum of [8] is present.

\subsection{General Analysis for the Large Volume Limit}

We now investigate the topological conditions on an arbitrary Calabi-Yau three-fold under which the scalar potential (2.9) admits an AdS non-supersymmetric minimum at exponentially large volume deepening the analysis performed in [8]. We will refer to those constructions as LARGE Volume Scenarios (LVS). ${ }^{1}$

\footnotetext{
${ }^{1}$ The capitalisation of LARGE is a reminder that the volume is exponentially large, and not just large enough to trust the supergravity limit.
} 
Claim 1 (LARGE Volume) Let $X$ be a Calabi-Yau three-fold and let the large volume limit be taken in the following way:

$$
\left\{\begin{array}{c}
\tau_{j} \text { remains small, } \forall j=1, \ldots, N_{\text {small }}, \\
\mathcal{V} \rightarrow \infty \text { for } \tau_{j} \rightarrow \infty, \forall j=N_{\text {small }}+1, \ldots, h_{1,1}(X),
\end{array}\right.
$$

within type IIB $\mathcal{N}=14 D$ supergravity where the Kähler potential and the superpotential in Einstein frame take the form:

$$
\left\{\begin{array}{l}
K=K_{c s}-2 \ln (\mathcal{V}+\hat{\xi}), \\
W=W_{0}+\sum_{j=1}^{N_{\text {small }}} A_{j} e^{-a_{j} T_{j}} .
\end{array}\right.
$$

Then the scalar potential admits a set $H$ of AdS non-supersymmetric minima at exponentially large volume located at $\mathcal{V} \sim e^{a_{j} \tau_{j}} \forall j=1, \ldots, N_{\text {small }}$ if and only if $h_{2,1}(X)>$ $h_{1,1}(X)>1$, i.e. $\xi>0$ and $\tau_{j}$ is a local blow-up mode resolving a given point-like singularity $\forall j=1, \ldots, N_{\text {small }}$. In this case

$$
\left\{\begin{array}{c}
\text { if } h_{1,1}(X)=N_{\text {small }}+1, H=\{\text { a point }\} \\
\text { if } h_{1,1}(X)>N_{\text {small }}+1, H=\left\{\left(h_{1,1}(X)-N_{\text {small }}-1\right) \text { flat directions }\right\} .
\end{array}\right.
$$

The proof of the previous Claim is presented in appendix A where we show also that $\tau_{j}$ is the only blow-up mode resolving a point-like singularity if and only if $K_{j j}^{-1} \sim \mathcal{V} \sqrt{\tau_{j}}$. On the contrary when the same singularity is resolved by several independent blow-ups, say $\tau_{1}$ and $\tau_{2}$, then $K_{11}^{-1} \sim \mathcal{V} h_{1 / 2}^{(1)}\left(\tau_{1}, \tau_{2}\right)$ and $K_{22}^{-1} \sim \mathcal{V} h_{1 / 2}^{(2)}\left(\tau_{1}, \tau_{2}\right)$ with $h_{1 / 2}^{(j)}$ homogeneous function of degree $1 / 2$ such that $\frac{\partial^{2} h^{(j)}}{\partial \tau_{1} \partial \tau_{2}} \neq 0 \forall j=1,2$.

Let us now explain schematically the global picture of LVS for arbitrary Calabi-Yau manifolds according to the LARGE Volume Claim:

1. The Euler number of the Calabi Yau manifold must be negative. More precisely: $h_{12}>h_{11}>1$. This means that the coefficient $\hat{\xi}$ must be positive in order to guarantee that in a particular direction the potential goes to zero at infinity from below [8]. This is a both sufficient and necessary condition.

2. The Calabi-Yau manifold must have at least one blow-up mode corresponding to a 4-cycle modulus that resolves a point-like singularity. The associated modulus must have an induced non-perturbative superpotential. This is usually guaranteed since these cycles are rigid cycles of arithmetic genus one, which is precisely the condition needed for the existence of non-perturbative superpotentials in the flux-less case [4].

3. This 4-cycle, together with other blow-up modes possibly present, are fixed small by the interplay of non-perturbative and $\alpha^{\prime}$ corrections, which stabilise also the overall volume mode. Here small means larger than the string scale but not exponentially large unlike the volume. 
4. All the other 4-cycles, such as those corresponding to fibrations, cannot be stabilised small even though they may have induced non-perturbative effects. They are sent large making their non-perturbative corrections negligible.

5. At this stage, non blow-up Kähler moduli, except the overall volume mode, remain unfixed giving rise to essentially flat directions.

6. It turns out then that in order to freeze these moduli, it is crucial to study string loop corrections as the leading term in a $g_{s}$ expansion will be dominant over any potential non-perturbative correction.

Notice that these are conditions to find exponentially large volume minima and our results hold for generic $\mathcal{O}(1)$ values of $W_{0}$. There may exist other minima which do not have exponentially large volume for which our results do not have anything to say. For example, $\left|W_{0}\right| \ll 1$ may give rise to KKLT-like minima.

Summarising, if there are $N_{\text {small }}$ blow-up modes and $L=\left(h_{11}-N_{\text {small }}-1\right)$ modes which do not blow-up point-like singularities nor correspond to the overall modulus, then our results state that all the $N_{\text {small }}$ can be fixed at values large with respect to the string scale but not exponentially large, the overall volume is exponentially large and the other $L$ Kähler moduli are not fixed by these effects.

In reality, the directions corresponding to the non blow-up modes, if they have nonperturbative effects, are not rigorously flat, but according to our approximations this is a subtlety which is not important. The reason is that, as we will see in the next sections, those directions will be lifted by the inclusion of string loop corrections which are always dominant with respect to the non-perturbative ones.

We would also like to stress that the previous general picture shows how we need non-perturbative effects only in the blow-up modes to get an exponentially large volume minimum. As blow-up modes correspond to rigid exceptional divisors, the corresponding non-perturbative corrections will be generally present even in the fluxless case [4]. They can arise from either gaugino condensation of the gauge theory living on the stack of branes wrapping that 4-cycle or from Euclidean D3 brane instantons. On the contrary, it is not clear if all the other cycles can indeed get non-perturbative corrections to $W$, but this is not necessary to obtain LARGE Volume.

\section{Particular Examples}

Let us illustrate these results in a few explicit examples. At this stage we ignore string loop corrections but as we will show in section 1 and 5, these can in some cases actually be important and change the configuration of the system studied.

\subsection{The single-hole Swiss cheese: $\mathbb{C} P_{[1,1,1,6,9]}^{4}$}

The weighted projective space $\mathbb{C} P_{[1,1,1,6,9]}^{4}$ is the Calabi-Yau on which the LVS was originally realised. The overall volume in terms of 2 -cycle volumes is given by

$$
\mathcal{V}=\frac{1}{6}\left(3 t_{1}^{2} t_{5}+18 t_{1} t_{5}^{2}+36 t_{5}^{3}\right)
$$


The divisor volumes take the form $\tau_{4}=\frac{t_{1}^{2}}{2}, \tau_{5}=\frac{\left(t_{1}+6 t_{5}\right)^{2}}{2}$, from which it is immediate to see that

$$
\mathcal{V}=\frac{1}{9 \sqrt{2}}\left(\tau_{5}^{3 / 2}-\tau_{4}^{3 / 2}\right)
$$

$\xi$ is positive since $h_{1,1}<h_{2,1}$ and the limit (2.10) can be correctly performed with $\tau_{5} \rightarrow \infty$ and $\tau_{4}$ remaining small. Thus $N_{\text {small }}=1$ and we have to check if this case satisfies the condition of the LARGE Volume Claim which is $K_{44}^{-1} \simeq \mathcal{V} \sqrt{\tau_{4}}$. This is indeed satisfied as it can be seen either by direct calculation or by noticing that $\tau_{4}$ is a local blow-up. Omitting numerical factors, the scalar potential takes the form

$$
V \simeq \frac{\sqrt{\tau_{4}} e^{-2 a_{4} \tau_{4}}}{\mathcal{V}}-\frac{W_{0} \tau_{4} e^{-2 a_{4} \tau_{4}}}{\mathcal{V}^{2}}+\frac{\hat{\xi} W_{0}^{2}}{\mathcal{V}^{3}}
$$

As the $\mathbb{C} P_{[1,1,1,6,9]}^{4}$ example is a particular case of the LARGE Volume Claim, we conclude that the scalar potential (3.3) will admit an AdS minimum at exponentially large volume with $\left(h_{1,1}-N_{\text {small }}-1\right)=0$ flat directions. This is consistent with the original calculation in [8], which shows that the minimum is located at

$$
\left\langle\tau_{4}\right\rangle \simeq(4 \hat{\xi})^{2 / 3}, \quad\langle\mathcal{V}\rangle \simeq \frac{\hat{\xi}^{1 / 3} W_{0}}{a_{4} A_{4}} e^{a_{4}\left\langle\tau_{4}\right\rangle}
$$

\subsection{The multiple-hole Swiss cheese: $\mathcal{F}_{11}$ and $\mathbb{C} P_{[1,3,3,3,5]}^{4}$}

It is straightforward to realise that the LARGE Volume Claim can be used to generalise the previous case by adding several blow-up modes resolving point-like singularities that will be stabilised small. In this case the overall volume looks like

$$
\mathcal{V}=\alpha\left(\tau_{\text {big }}^{3 / 2}-\sum_{i=1}^{N_{\text {small }}} \lambda_{i} \tau_{i}^{3 / 2}\right),
$$

where $\alpha$ and $\lambda_{i}$ are positive model-dependent parameters and the Calabi-Yau manifold presents a typical "Swiss cheese" shape. An explicit example is the Fano three-fold $\mathcal{F}_{11}$ described in [20], which is topologically a $\mathbb{Z}_{2}$ quotient of a $C Y_{3}$ with Hodge numbers $h_{1,1}=3, h_{2,1}=111$. The total volume of the $\mathcal{F}_{11}$ reads

$$
\mathcal{V}=\frac{t_{1}^{2} t_{2}}{2}+\frac{t_{1} t_{2}^{2}}{2}+\frac{t_{2}^{3}}{6}+\frac{t_{1}^{2} t_{3}}{2}+2 t_{1} t_{2} t_{3}+t_{2}^{2} t_{3}+t_{1} t_{3}^{2}+2 t_{2} t_{3}^{2}+\frac{2 t_{3}^{3}}{3}
$$

and the 4-cycle moduli are given by

$$
\tau_{1}=\frac{t_{2}}{2}\left(2 t_{1}+t_{2}+4 t_{3}\right), \quad \tau_{2}=\frac{t_{1}^{2}}{2}, \quad \tau_{3}=t_{3}\left(t_{1}+t_{3}\right) .
$$

It is then possible to express $\mathcal{V}$ in terms of the $\tau$-moduli as

$$
\mathcal{V}=\frac{1}{3 \sqrt{2}}\left(2\left(\tau_{1}+\tau_{2}+2 \tau_{3}\right)^{3 / 2}-\left(\tau_{2}+2 \tau_{3}\right)^{3 / 2}-\tau_{2}^{3 / 2}\right)
$$

The resemblance with the general "Swiss cheese" picture (3.5) is now manifest. Two further Calabi-Yau realisations of this Swiss-Cheese structure have been presented in [14]. They 
are the $h_{1,1}=3$ degree 15 hypersurface embedded in $\mathbb{C} P_{[1,3,3,3,5]}^{4}$ and the $h_{1,1}=5$ degree 30 hypersurface in $\mathbb{C} P_{[1,1,3,10,15]}^{4}$. All these many moduli "Swiss cheese" Calabi-Yaus will admit a LARGE Volume minimum located at

$$
\langle\mathcal{V}\rangle \sim e^{a_{i}\left\langle\tau_{i}\right\rangle}, \quad \forall i=1, \ldots, N_{\text {small }},
$$

with no orthogonal flat directions. Let us briefly review the geometric data of the resolution of the $\mathbb{C} P_{[1,3,3,3,5]}^{4}$ manifold [14], since it turns out to be an interesting case in which loop corrections may potentially stabilise the Standard Model cycle that does not admit nonperturbative superpotential contributions. In the diagonal basis the total volume becomes

$$
\mathcal{V}=\sqrt{\frac{2}{45}}\left(\tau_{a}^{3 / 2}-\frac{1}{3} \tau_{b}^{3 / 2}-\frac{\sqrt{5}}{3} \tau_{c}^{3 / 2}\right)
$$

A Euclidean D3-brane instanton wraps the rigid 4-cycle $D_{E 3}=\frac{1}{3}\left(D_{b}+D_{c}\right)$, giving a non-perturbative superpotential term $W_{n p}=e^{-\frac{2 \pi}{3}\left(\tau_{b}+\tau_{c}\right)}$. There are also two stacks of D7-branes wrapping the rigid four cycles $D_{D 7 A}=\frac{1}{3}\left(D_{b}-2 D_{c}\right)$ and $D_{D 7 B}=D_{c}$ with line bundles $\mathcal{L}_{A}=\frac{1}{3}\left(2 D_{b}+5 D_{c}\right)$ and $\mathcal{L}_{B}=\mathcal{O}$. This choice guarantees that there are no chiral zero modes on the D7-E3 intersections. The "Standard Model" is part of the $U\left(N_{A}\right)$ gauge group on the stack $A$ of D7-branes, with SM matter obtained from the intersections $A A^{\prime}$ and $A B$ where the prime denotes the orientifold image.

Neglecting the D-term part of the scalar potential we obtain

$$
V=\frac{\lambda_{1}\left(\sqrt{5 \tau_{b}}+\sqrt{\tau_{c}}\right) e^{-\frac{4 \pi}{3}\left(\tau_{b}+\tau_{c}\right)}}{\mathcal{V}}-\frac{\lambda_{2}\left(\tau_{b}+\tau_{c}\right) e^{-\frac{2 \pi}{3}\left(\tau_{b}+\tau_{c}\right)}}{\mathcal{V}^{2}}+\frac{\lambda_{3}}{\mathcal{V}^{3}}
$$

where $\lambda_{i}>0, \forall i=1,2,3$ are unimportant numerical factors. Now to make the study of the scalar potential (3.11) simpler, we perform the change of coordinates $\tau_{b}=2 \tau_{E 3}+\tau_{S M}, \tau_{c}=$ $\tau_{E 3}-\tau_{S M}$, bringing (3.11) to the form

$$
V=\frac{\lambda_{1}\left(\sqrt{5\left(2 \tau_{E 3}+\tau_{S M}\right)}+\sqrt{\tau_{E 3}-\tau_{S M}}\right) e^{-4 \pi \tau_{E 3}}}{\mathcal{V}}-\frac{3 \lambda_{2} \tau_{E 3} e^{-2 \pi \tau_{E 3}}}{\mathcal{V}^{2}}+\frac{\lambda_{3}}{\mathcal{V}^{3}} .
$$

The scalar potential (3.12) then has a critical point at $\tau_{E 3}=2 \tau_{S M}$. However, this is not a minimum of the full scalar potential but is actually a saddle point along $\tau_{S M}$ at fixed $\tau_{E 3}$ and $\mathcal{V}$. In subsection 5.2 we will show how string loop corrections may give rise to a stable LVS even though no non-perturbative corrections in $\tau_{S M}$ are included (see [14 for a discussion of freezing $\tau_{S M}$ by including D-terms with (3.12)).

\subsection{2-Parameter K3 Fibration: $\mathbb{C} P_{[1,1,2,2,6]}^{4}$}

Our next example is a fibration Calabi-Yau, $\mathbb{C} P_{[1,1,2,2,6]}^{4}$. The overall volume in terms of 2 -cycle volumes is given by

$$
\mathcal{V}=t_{1} t_{2}^{2}+\frac{2}{3} t_{2}^{3}
$$

The 4-cycle volumes take the form $\tau_{1}=t_{2}^{2}, \tau_{2}=2 t_{2}\left(t_{1}+t_{2}\right)$, yielding

$$
\mathcal{V}=\frac{1}{2} \sqrt{\tau_{1}}\left(\tau_{2}-\frac{2}{3} \tau_{1}\right)
$$


It is possible to invert the relations $\tau_{i}=\partial \mathcal{V} / \partial t_{i}$ to produce

$$
t_{2}=\sqrt{\tau_{1}}, \quad t_{1}=\frac{\tau_{2}-2 \tau_{1}}{2 \sqrt{\tau_{1}}} .
$$

The Euler characteristic of the Calabi-Yau is negative and the limit (2.10) can be performed only with $\tau_{2} \rightarrow \infty$ and keeping $\tau_{1}$ small. This corresponds to $t_{1} \rightarrow \infty$ and $t_{2}$ small. In this limit the volume becomes

$$
\mathcal{V}=\frac{1}{2} \sqrt{\tau_{1}} \tau_{2} \simeq t_{1} t_{2}^{2} \simeq t_{1} \tau_{1}
$$

Thus $N_{\text {small }}=1$ again and we need to check the condition of the LARGE Volume Claim: $K_{11}^{-1} \simeq \mathcal{V} \sqrt{\tau_{1}}$. However this is clearly not satisfied, as $\tau_{1}$ is a fibration over the base $t_{1}$.

This is therefore a situation where no exponentially large volume minimum is present, as can be confirmed by the explicit calculation below.

\subsubsection{Explicit Calculation}

Here we verify that the $\mathbb{C} P_{[1,1,2,2,6]}^{4}$ model does not give a realisation of the LVS. We take the large volume limit in the following way

$$
\left\{\begin{array}{c}
\tau_{1} \text { small, } \\
\tau_{2} \gg 1,
\end{array}\right.
$$

which, after the axion minimisation $\left(W_{0}>0\right)$, gives a scalar potential of the form

$$
\begin{aligned}
V= & V_{n p}+V_{\alpha^{\prime}}=\frac{4}{\mathcal{V}^{2}}\left[a_{1} A_{1}^{2} \tau_{1}\left(a_{1} \tau_{1}+1\right) e^{-2 a_{1} \tau_{1}}-a_{1} A_{1} \tau_{1} W_{0} e^{-a_{1} \tau_{1}}\right] \\
& +\frac{3}{4} \frac{\xi}{\mathcal{V}^{3}}\left(W_{0}^{2}+A_{1}^{2} e^{-2 a_{1} \tau_{1}}-2 A_{1} W_{0} e^{-a_{1} \tau_{1}}\right) .
\end{aligned}
$$

We set $A_{1}=1$ and recall that to neglect higher order instanton corrections we need $a_{1} \tau_{1} \gg 1$. (3.18) becomes

$$
V=\frac{4}{\mathcal{V}^{2}}\left[\left(a_{1} \tau_{1} e^{-a_{1} \tau_{1}}-W_{0}\right) a_{1} \tau_{1} e^{-a_{1} \tau_{1}}\right]+\frac{3}{4} \frac{\xi}{\mathcal{V}^{3}}\left[W_{0}^{2}+\left(e^{-a_{1} \tau_{1}}-2 W_{0}\right) e^{-a_{1} \tau_{1}}\right] .
$$

The previous expression (3.19) can be rewritten as

$$
\begin{gathered}
V=\frac{e^{-2 a_{1} \tau_{1}}}{\mathcal{V}^{2}}\left(4 a_{1}^{2} \tau_{1}^{2}+\frac{3}{4} \frac{\xi}{\mathcal{V}}\right)-\frac{2 W_{0} e^{-a_{1} \tau_{1}}}{\mathcal{V}^{2}}\left(2 a_{1} \tau_{1}+\frac{3}{4} \frac{\xi}{\mathcal{V}}\right)+\frac{3}{4} \frac{\xi}{\mathcal{V}^{3}} W_{0}^{2} \\
\sim \widetilde{\mathcal{V} \gg 1} \frac{4}{\mathcal{V}^{2}}\left[\left(a_{1} \tau_{1} e^{-a_{1} \tau_{1}}-W_{0}\right) a_{1} \tau_{1} e^{-a_{1} \tau_{1}}\right]+\frac{3}{4} \frac{\xi}{\mathcal{V}^{3}} W_{0}^{2} .
\end{gathered}
$$

Assuming a natural value $W_{0} \sim \mathcal{O}(1)$, then (3.20) simplifies to

$$
V=-\frac{4}{\mathcal{V}^{2}} W_{0} a_{1} \tau_{1} e^{-a_{1} \tau_{1}}+\frac{3}{4} \frac{\xi}{\mathcal{V}^{3}} W_{0}^{2} .
$$

Extremising this scalar potential, we get

$$
\frac{\partial V}{\partial \tau_{1}}=\frac{4}{\mathcal{V}^{2}} W_{0} a_{1} e^{-a_{1} \tau_{1}}\left(a_{1} \tau_{1}-1\right)=0,
$$


whose only possible solution for $W_{0} \neq 0$ is $a_{1} \tau_{1}=1$, which is not in the controlled regime of parameter space. However, when $W_{0}=0$, (3.20) gives

$$
V=\left(4 a_{1}^{2} \tau_{1}^{2}+\frac{3}{4} \frac{\xi}{\mathcal{V}}\right) \frac{e^{-2 a_{1} \tau_{1}}}{\mathcal{V}^{2}} \underset{\mathcal{V} \gg 1}{\sim} 4 a_{1}^{2} \tau_{1}^{2} \frac{e^{-2 a_{1} \tau_{1}}}{\mathcal{V}^{2}}
$$

and the first derivative with respect to $\tau_{1}$ is

$$
\frac{\partial V}{\partial \tau_{1}}=8 a_{1}^{2} \tau_{1} \frac{e^{-2 a_{1} \tau_{1}}}{\mathcal{V}^{2}}\left(a_{1} \tau_{1}-1\right)
$$

which also has no minimum. Thus we have shown that for $W_{0} \sim \mathcal{O}(1)$, the $\mathbb{C} P_{[1,1,2,2,6]}^{4}$ model has no exponentially large volume minimum. The last hope is to find a minimum fine tuning $W_{0} \ll 1$. In this case taking the derivatives of (3.20), one obtains

$$
\frac{\partial V}{\partial \tau_{1}}=\frac{4}{\mathcal{V}^{2}} a_{1} e^{-2 a_{1} \tau_{1}}\left(a_{1} \tau_{1}-1\right)\left(W_{0} e^{a_{1} \tau_{1}}-2 a_{1} \tau_{1}\right)=0,
$$

whose only possible solution is

$$
2 a_{1}\left\langle\tau_{1}\right\rangle=W_{0} e^{a_{1}\left\langle\tau_{1}\right\rangle}
$$

but then fixing $\tau_{1}$, the scalar potential (3.20) along the volume direction looks like

$$
V \sim \frac{W_{0}^{2}}{\mathcal{V}^{2}}\left(-1+\frac{3}{4} \frac{\xi}{\mathcal{V}}\right) \sim-\frac{W_{0}^{2}}{\mathcal{V}^{2}}
$$

The potential (3.27) has no LARGE Volume minimum and so we conclude that the $\mathbb{C} P_{[1,1,2,2,6]}^{4}$ model does not admit an exponentially large volume minimum for any value of $W_{0}$.

It is still of course possible to fix the moduli using other stabilisation schemes - for example KKLT. However, in this case there will not be a large hierarchy between the two Kähler moduli, with instead $\tau_{1} \lesssim \tau_{2}$, and the volume can never be exponentially large.

\subsection{3-Parameter K3 Fibration}

In the previous Sections 3.1, 3.2 and 3.3, we have presented three examples which illustrate two of the three possible situations which the general analysis determines. We now illustrate the case when an exponentially large volume minimum can be found, but with flat directions still present. We will then explain how these can be lifted using string loop corrections.

This example concerns Calabi-Yau three-folds which are single K3 Fibrations with three Kähler moduli. We start off with the following expression for the overall volume in terms of the three moduli

$$
\mathcal{V}=\alpha\left[\sqrt{\tau_{1}}\left(\tau_{2}-\beta \tau_{1}\right)-\gamma \tau_{3}^{3 / 2}\right]
$$

where $\alpha, \beta, \gamma$ are positive model-dependent constants. While we do not have any explicit realisation of such kind of Calabi-Yau manifold, eq. (3.28) is simply the expression for the $\mathbb{C} P_{[1,1,2,2,6]}^{4}$ case (3.14), augmented by the inclusion of a blow-up mode $\tau_{3}$. We also assume that $h_{2,1}(X)>h_{1,1}(X)=3$, thus satisfying the other condition of the LARGE Volume Claim. There are then two ways to perform the limit (2.10) without obtaining an internal volume that is formally negative: 
1.

$$
\left\{\begin{array}{l}
\tau_{i} \rightarrow \infty, \quad \forall i=1,2 \text { with the constraint } \tau_{1}<\tau_{2}, \\
\tau_{3} \text { remains small. }
\end{array}\right.
$$

This case keeps both cycles associated with the fibration large, while the blow-up cycle remains small. Given that $\tau_{1} \rightarrow \infty$, this situation resembles the "Swiss cheese" picture

$$
\mathcal{V}=\alpha[\underbrace{\sqrt{\tau_{1}}\left(\tau_{2}-\beta \tau_{1}\right)}_{\tau_{b i g}^{3 / 2}}-\gamma \tau_{3}^{3 / 2}],
$$

and due to this analogy with the $\mathbb{C} P_{[1,1,1,6,9]}^{4}$ model, the condition $K_{33}^{-1} \simeq \mathcal{V} \sqrt{\tau_{3}}$ is verified. Thus we can apply the LARGE Volume Claim which states that the scalar potential will have an AdS exponentially large volume set of minima together with $\left(h_{1,1}-N_{\text {small }}-1\right)=1$ flat directions. In the following section we shall confirm this with an explicit calculation.

2.

$$
\left\{\begin{array}{l}
\tau_{2} \rightarrow \infty \\
\tau_{1} \text { and } \tau_{3} \text { remain small. }
\end{array}\right.
$$

In this case $N_{\text {small }}=2$ and according to the LARGE Volume Claim there will be an exponentially large volume minimum of the scalar potential if and only if both $\tau_{1}$ and $\tau_{3}$ is a blow-up mode. As we show in the next Section 3.4.1, $K_{33}^{-1} \sim \mathcal{V} \sqrt{\tau_{3}}$, as is suggested by the volume form $(3.28)$. However $K_{11}^{-1} \sim \tau_{1}^{2}$, as could be guessed from the fact that the overall volume (3.13) in terms of the 2-cycles moduli is linear in $t_{1}$. Hence $\tau_{1}$ is not a blow-up but a fibration modulus that does not give rise to LVS.

We now confirm these statements with explicit calculations.

\subsubsection{Explicit Calculation}

We focus on the case in which

$$
\mathcal{V}=\alpha\left[\sqrt{\tau_{1}}\left(\tau_{2}-\beta \tau_{1}\right)-\gamma \tau_{3}^{3 / 2}\right]
$$

where $\alpha, \beta, \gamma$ are positive model-dependent constants and the Kähler potential and the superpotential take the form (defining $\hat{\xi} \equiv \xi g_{s}^{-3 / 2}$ ):

$$
\begin{gathered}
K=K_{0}+\delta K_{\left(\alpha^{\prime}\right)}=-2 \ln \left(\mathcal{V}+\frac{\hat{\xi}}{2}\right), \\
W=W_{0}+A_{1} e^{-a_{1} T_{1}}+A_{2} e^{-a_{2} T_{2}}+A_{3} e^{-a_{3} T_{3}} .
\end{gathered}
$$

In the large volume limit the Kähler matrix and its inverse look like:

$$
K_{i j}^{0}=\frac{1}{4 \mathcal{V}^{2}}\left(\begin{array}{ccc}
\frac{\mathcal{V}^{2}}{\tau_{1}^{2}}+2 \alpha^{2} \beta^{2} \tau_{1} & \frac{\alpha^{2}}{\sqrt{\tau_{1}}}\left(\gamma \tau_{3}^{3 / 2}-2 \beta \tau_{1}^{3 / 2}\right) & \frac{3 \alpha \gamma}{2} \frac{\sqrt{\tau_{3}}}{\tau_{1}}\left(2 \alpha \beta \tau_{1}^{3 / 2}-\mathcal{V}\right) \\
\frac{\alpha^{2}}{\sqrt{\tau_{1}}}\left(\gamma \tau_{3}^{3 / 2}-2 \beta \tau_{1}^{3 / 2}\right) & 2 \alpha^{2} \tau_{1} & -3 \alpha^{2} \gamma \sqrt{\tau_{1}} \sqrt{\tau_{3}} \\
\frac{3 \alpha \gamma}{2} \frac{\sqrt{\tau_{3}}}{\tau_{1}}\left(2 \alpha \beta \tau_{1}^{3 / 2}-\mathcal{V}\right) & -3 \alpha^{2} \gamma \sqrt{\tau_{1}} \sqrt{\tau_{3}} & \frac{3 \alpha \gamma}{2} \frac{\mathcal{V}}{\sqrt{\tau_{3}}}
\end{array}\right),
$$


and

$$
K_{0}^{i j}=4\left(\begin{array}{ccc}
\tau_{1}^{2} & \beta \tau_{1}^{2}+\gamma \sqrt{\tau_{1}} \tau_{3}^{3 / 2} & \tau_{1} \tau_{3} \\
\beta \tau_{1}^{2}+\gamma \sqrt{\tau_{1}} \tau_{3}^{3 / 2} & \frac{\mathcal{V}^{2}}{2 \alpha^{2} \tau_{1}}+\beta^{2} \tau_{1}^{2} & \tau_{3}\left(\frac{\mathcal{V}}{\alpha \sqrt{\tau_{1}}}+\beta \tau_{1}\right) \\
\tau_{1} \tau_{3} & \tau_{3}\left(\frac{\mathcal{V}}{\alpha \sqrt{\tau_{1}}}+\beta \tau_{1}\right) & \frac{2}{3 \alpha \gamma} \mathcal{V} \sqrt{\tau_{3}}
\end{array}\right)
$$

Both of the ways outlined above to take the large volume limit have $\tau_{2} \gg 1$ and so the superpotential (3.34) can be simplified as follows:

$$
W \simeq W_{0}+A_{1} e^{-a_{1} T_{1}}+A_{3} e^{-a_{3} T_{3}} .
$$

The scalar potential takes its general form (2.9). In the large volume limit, the $\alpha^{\prime}$ leading contribution to the scalar potential becomes

$$
V_{(\alpha \prime)}=3 e^{K} \hat{\xi} \frac{\left(\hat{\xi}^{2}+7 \hat{\xi} \mathcal{V}+\mathcal{V}^{2}\right)}{(\mathcal{V}-\hat{\xi})(2 \mathcal{V}+\hat{\xi})^{2}}|W|^{2} \underset{\mathcal{V} \rightarrow \infty}{\longrightarrow} \frac{3}{4} \frac{\hat{\xi}}{\mathcal{V}^{3}}|W|^{2}
$$

Adding this to the non-perturbative part, we are left with

$$
\begin{aligned}
V= & \frac{1}{\mathcal{V}^{2}}\left[K_{0}^{i j} a_{i} A_{i} a_{j} \bar{A}_{j} e^{-\left(a_{i} T_{i}+a_{j} \bar{T}_{j}\right)}+2 a_{i} A_{i} \tau_{i} e^{-a_{i} T_{i}} \bar{W}\right. \\
& \left.+2 a_{j} \bar{A}_{j} \tau_{j} e^{-a_{j} \bar{T}_{j}} W\right]+\frac{3}{4} \frac{\hat{\xi}}{\mathcal{V}^{3}}|W|^{2} .
\end{aligned}
$$

We shall focus on the case $W_{0} \sim \mathcal{O}(1)$, (since a tuned small value of $W_{0}$ cannot give rise to large volume) and for $a_{1} \tau_{1} \gg 1, a_{3} \tau_{3} \gg 1$, after extremising the axion directions, the scalar potential simplifies to (with $\lambda \equiv 8 /(3 \alpha \gamma), \nu \equiv 3 \hat{\xi} / 4$ and $A_{1}=A_{3}=1$ )

$$
V=\frac{\lambda a_{3}^{2}}{\mathcal{V}} \sqrt{\tau_{3}} e^{-2 a_{3} \tau_{3}}-\frac{4}{\mathcal{V}^{2}} W_{0} a_{1} \tau_{1} e^{-a_{1} \tau_{1}}-\frac{4}{\mathcal{V}^{2}} W_{0} a_{3} \tau_{3} e^{-a_{3} \tau_{3}}+\frac{\nu}{\mathcal{V}^{3}} W_{0}^{2}
$$

The scalar potential $V$ depends on $\mathcal{V}, \tau_{1}$ and $\tau_{3}: V=V\left(\mathcal{V}, \tau_{1}, \tau_{3}\right)$, with the dependence on $\tau_{2}$ implicit in the internal volume $\mathcal{V}$. The large volume limit can be taken in the two ways (1 and 2) outlined in the previous section 3.4. The difference between these two cases is that in limit $1 \tau_{1} \rightarrow \infty$ whereas in limit $2 \tau_{1}$ remains small. Let us now study these two different cases in detail.

1) $\tau_{1} \rightarrow \infty \Leftrightarrow \tau_{3} \ll \tau_{1}<\tau_{2}$

In this case, the superpotential (3.37) obtains non-perturbative corrections only in $\tau_{3}$ :

$$
W \simeq W_{0}+A_{3} e^{-a_{3} T_{3}} .
$$

Since the $A_{1}$ term is not present in (3.41), we will be unable to stabilise the corresponding Kähler modulus $\tau_{1}$, thereby giving rise to an exactly flat direction. In this case the scalar potential (3.40) further reduces to:

$$
V=\frac{\lambda a_{3}^{2}}{\mathcal{V}} \sqrt{\tau_{3}} e^{-2 a_{3} \tau_{3}}-\frac{4}{\mathcal{V}^{2}} W_{0} a_{3} \tau_{3} e^{-a_{3} \tau_{3}}+\frac{\nu}{\mathcal{V}^{3}} W_{0}^{2}
$$


and $V$ depends only on $\mathcal{V}$ and $\tau_{3}: V=V\left(V, \tau_{3}\right)$. The potential (3.42) has the same form as the scalar potential found in section 3.2 of [8] where the $\mathbb{C} P_{[1,1,1,6,9]}^{4}$ case was first discussed. Following the same reasoning, we look for possible minima of the scalar potential (3.42) by working out the two minimisation conditions:

$$
\begin{gathered}
\frac{\partial V}{\partial \mathcal{V}}=0 \quad \Leftrightarrow \quad\left(\lambda a_{3}^{2} \sqrt{\tau_{3}} e^{-2 a_{3} \tau_{3}}\right) \mathcal{V}^{2}-\left(8 W_{0} a_{3} \tau_{3} e^{-a_{3} \tau_{3}}\right) \mathcal{V}+3 \nu W_{0}^{2}=0, \\
\frac{\partial V}{\partial \tau_{3}}=0 \quad \Leftrightarrow \quad \frac{\lambda a_{3}}{2 \sqrt{\tau_{3}}} \mathcal{V} e^{-a_{3} \tau_{3}}\left(1-4 a_{3} \tau_{3}\right)+4 W_{0}\left(a_{3} \tau_{3}-1\right)=0 .
\end{gathered}
$$

Equation (3.43) admits a solution of the form

$$
\frac{\lambda a_{3}}{4 W_{0} \sqrt{\tau_{3}}} \mathcal{V} e^{-a_{3} \tau_{3}}=1 \pm \sqrt{1-\frac{3 \lambda \nu}{16 \tau_{3}^{3 / 2}}}
$$

whereas in the approximation $a_{3} \tau_{3} \gg 1$, (3.44) becomes:

$$
\frac{\lambda a_{3}}{2 \sqrt{\tau_{3}}} \mathcal{V} e^{-a_{3} \tau_{3}}=W_{0}
$$

Combining (3.45) and (3.46), we find $\frac{1}{2}=1 \pm \sqrt{1-\frac{3 \lambda \nu}{16 \tau_{3}^{3 / 2}}}$, whose solution is given by

$$
\left\langle\tau_{3}\right\rangle=\frac{1}{g_{s}}\left(\frac{\xi}{2 \alpha \gamma}\right)^{2 / 3} \sim \frac{1}{g_{s}} .
$$

On the contrary, from (3.46) we work out

$$
\langle\mathcal{V}\rangle=\frac{3(\alpha \gamma)^{2 / 3} W_{0}}{4 a_{3} \sqrt{g_{s}}}\left(\frac{\xi}{2}\right)^{1 / 3} e^{\frac{a_{3}}{g_{s}}\left(\frac{\xi}{2 \alpha \gamma}\right)^{2 / 3}} \sim \frac{W_{0}}{a_{3} \sqrt{g_{s}}} e^{\frac{a_{3}}{g_{s}}} .
$$

There is therefore an exponentially large volume minimum. Setting $\alpha=\gamma=1, \xi=2$, $g_{s}=0.1, a_{3}=\pi$ and $W_{0}=1$, we finally obtain $\left\langle\tau_{3}\right\rangle=10$ and $\langle\mathcal{V}\rangle=3.6 \cdot 10^{13}$. However there is still the presence of an exactly flat direction which can be better appreciated after the following change of coordinates:

$$
\left(\tau_{1}, \tau_{2}\right) \quad \longrightarrow \quad(\mathcal{V}, \Omega): \quad\left\{\begin{array}{l}
\mathcal{V} \simeq \alpha\left[\sqrt{\tau_{1}}\left(\tau_{2}-\beta \tau_{1}\right)\right] \\
\Omega=\alpha\left[\sqrt{\tau_{1}}\left(\tau_{2}+\beta \tau_{1}\right)\right]
\end{array}\right.
$$

From (3.48) and (3.49) we see that the stabilisation of $\mathcal{V}$ and $\tau_{3}$ does not depend on $\Omega$ at all, implying that $\Omega$ is a flat direction. We plot below in Figure 2 the behaviour of this scalar potential where the flat direction is manifest: $\tau_{3}$ has been already fixed as $\left\langle\tau_{3}\right\rangle=10$, $\mathcal{V}$ is plotted on the $x$-axis and $\Omega$ on the $y$-axis.

\section{2) $\tau_{1}$ small}

In this case the large volume limit is taken keeping $\tau_{1}$ small and the scalar potential takes the general form (3.40). The minimisation equation with respect to $\tau_{1}$ reads

$$
\frac{\partial V}{\partial \tau_{1}}=0 \quad \Leftrightarrow \quad \frac{4}{\mathcal{V}^{2}} W_{0} a_{1} e^{-a_{1} \tau_{1}}\left(a_{1} \tau_{1}-1\right)=0,
$$




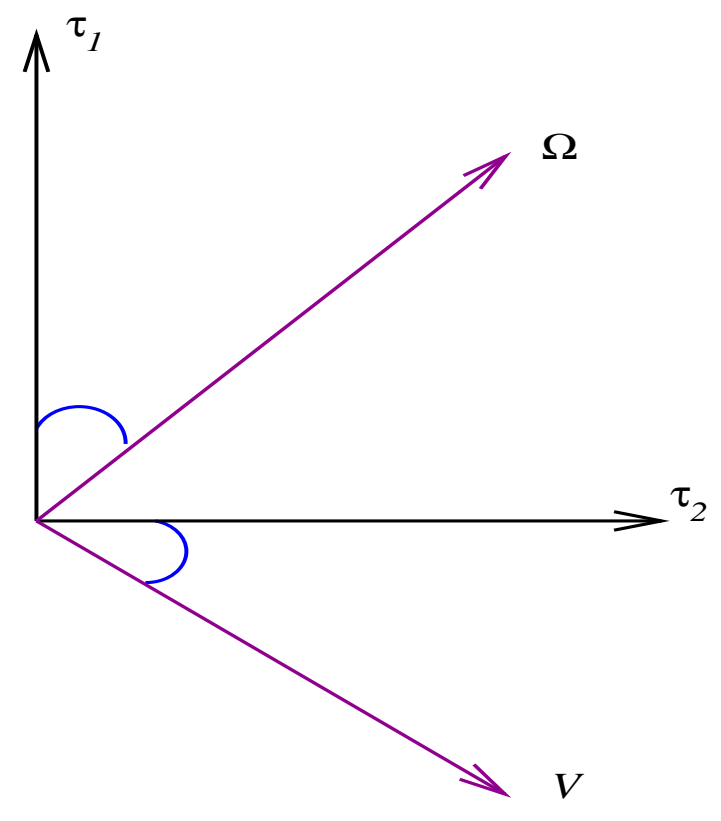

Figure 1: Change of coordinates of the Kähler moduli space.

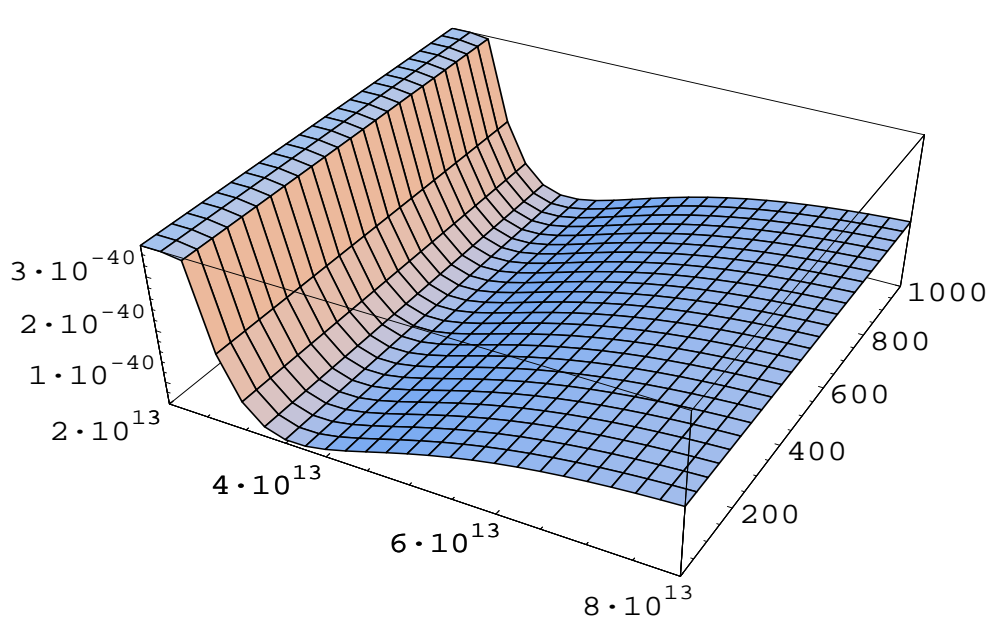

Figure 2: "Sofa" potential with the presence of a flat direction.

which implies $a_{1} \tau_{1}=1$ and so we cannot neglect higher instanton corrections.

There is therefore no trustable minimum for the $\tau_{1}$ field. We may however think about a situation in which the system still has an exponentially large internal volume, with $\tau_{3}$ and $\mathcal{V}$ sitting at their minimum $\langle\mathcal{V}\rangle \sim e^{a_{3}\left\langle\tau_{3}\right\rangle}$, while $\tau_{1}$ plays the rôle of a quintessence field rolling in a region at large $\tau_{1} \gg 1$ away from $a_{1} \tau_{1}=1$. The quintessence scale would be set by the $e^{-a_{1} \tau_{1}}$ exponent. Setting $a_{1} \tau_{1} \gg 1$ it is easy to see that this is possible. However, the values of $a_{1}$ and $\tau_{1}$ need to be tuned to get a realistically small mass for $\tau_{1}$ and even if 
this is done the fifth force problems of quintessence fields would seem to be unavoidable.

Finally, let us summarize in the table below the results found without string loop corrections to $K$.

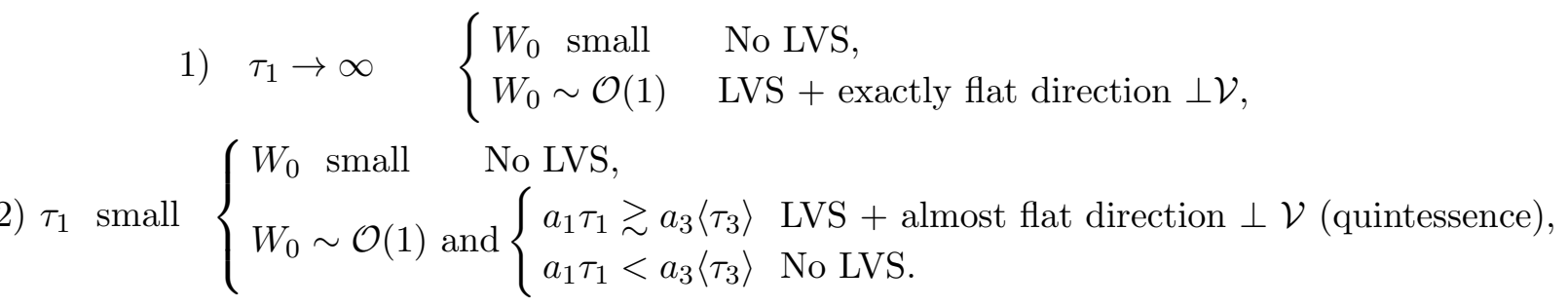

\section{Inclusion of the String Loop Corrections}

One of the purposes of this paper is to study the effect of string loop corrections on moduli stabilisation. On a Calabi-Yau it is of course not possible to explicitly determine the full functional form of loop corrections. Nonetheless, it may still be possible to extract the leading scaling behaviour with the moduli of the loop corrections, even if the detailed form of the prefactors cannot be determined. As the Kähler moduli are not stabilised at tree-level, even this leading scaling behaviour is very significant.

This is the philosophy that was pursued in [13, 12], where the expected parametric behaviour of the string loop corrections to the Kähler potential was studied, even if the detailed prefactors cannot be computed. On the torus, the explicit string computation of [10] does exist. For the $T^{6} /\left(\mathbb{Z}_{2} \times \mathbb{Z}_{2}\right)$ case the loop corrections take the form

$$
\delta K_{\left(g_{s}\right)}=\delta K_{\left(g_{s}\right)}^{K K}+\delta K_{\left(g_{s}\right)}^{W},
$$

where $\delta K_{\left(g_{s}\right)}^{K K}$ comes from the exchange between D7 and D3-branes of closed strings which carry Kaluza-Klein momentum, and reads (for vanishing open string scalars)

$$
\delta K_{\left(g_{s}\right)}^{K K}=-\frac{1}{128 \pi^{4}} \sum_{i=1}^{3} \frac{\mathcal{E}_{i}^{K K}(U, \bar{U})}{\operatorname{Re}(S) \tau_{i}} .
$$

The other correction $\delta K_{\left(g_{s}\right)}^{W}$ is interpreted in the closed string channel as due to exchange of winding strings between intersecting stacks of D7-branes. It takes the form

$$
\delta K_{\left(g_{s}\right)}^{W}=-\left.\frac{1}{128 \pi^{4}} \sum_{i=1}^{3} \frac{\mathcal{E}_{i}^{W}(U, \bar{U})}{\tau_{j} \tau_{k}}\right|_{j \neq k \neq i} .
$$

Note in both cases there is a very complicated dependence of the corrections on the $U$ moduli, encoded in the functions $\mathcal{E}_{i}(U, \bar{U})$, but a very simple dependence on the $T$ moduli.

These formulae were generalised by [13] for the behaviour of loop corrections on general Calabi-Yau three-folds. As a Weyl rescaling is always necessary to convert the string computation to Einstein frame, they proposed

$$
\delta K_{\left(g_{s}\right)}^{K K} \sim \sum_{i=1}^{h_{1,1}} \frac{\mathcal{C}_{i}^{K K}(U, \bar{U})\left(a_{i l} t^{l}\right)}{\operatorname{Re}(S) \mathcal{V}},
$$


where $a_{l} t^{l}$ is a linear combination of the basis 2-cycle volumes $t_{l}$. A similar line of argument for the winding corrections gives

$$
\delta K_{\left(g_{s}\right)}^{W} \sim \sum_{i=1}^{h_{1,1}} \frac{\mathcal{C}_{i}^{W}(U, \bar{U})}{\left(a_{i l} t^{l}\right) \mathcal{V}}
$$

$\mathcal{C}_{i}^{K K}$ and $\mathcal{C}_{i}^{W}$ are unknown functions of the complex structure moduli; however, as the complex structure moduli are flux-stabilised these reduce to an (unknown) constant. This approach is therefore useful to fix the leading order dependence on Kähler moduli.

In [12] the current authors showed that the proposed form of these correction is consistent with what would be expected based on the form of the Coleman-Weinberg potential in supergravity [22, 23],

$$
\delta V_{1-\text { loop }} \sim \Lambda^{4} S \operatorname{Tr}\left(M^{0}\right)+2 \Lambda^{2} S \operatorname{Tr}\left(M^{2}\right)+S \operatorname{Tr}\left(M^{4} \ln \left(\frac{M^{2}}{\Lambda^{2}}\right)\right),
$$

where $\Lambda$ denotes the cut-off scale and $\operatorname{STr}\left(M^{n}\right)$ is the supertrace. The cut-off scale is taken to be the scale at which the higher supersymmetry of the ten-dimensional theory becomes apparent, effectively giving an extended supersymmetry within the loops.

In terms of the corrections to the Kähler potential, the leading contribution of these corrections to the scalar potential is always vanishing, giving an "extended no-scale structure". This result holds in general as long as the corrections are homogeneous functions of degree -2 in the 2-cycle volumes.

In [12] a general formula was also worked out for the first non-vanishing contribution to the effective scalar potential of $\delta K_{\left(g_{s}\right)}$ as given by the conjectures (4.4)-(4.5). It turns out to be relatively simple and expressible in terms of the tree-level Kähler metric $K_{0}=-2 \ln (\mathcal{V})$ and the winding correction to the Kähler potential:

$$
\delta V_{\left(g_{s}\right)}^{1-\text { loop }}=\sum_{i=1}^{h_{1,1}}\left(\frac{\left(\mathcal{C}_{i}^{K K}\right)^{2}}{\operatorname{Re}(S)^{2}} K_{i i}^{0}-2 \delta K_{\left(g_{s}\right), \tau_{i}}^{W}\right) \frac{W_{0}^{2}}{\mathcal{V}^{2}} .
$$

The low energy interpretation of the 1-loop scalar potential (4.7) comes from matching it with the Coleman-Weinberg potential (4.6). The leading term in both cases vanishes, and so the comparison should involve the leading non-zero terms in both cases. These match precisely for the various cases studied in [12].

This low-energy interpretation can easily be illustrated in the simple one-modulus case where the volume takes the form $\mathcal{V}=\tau^{3 / 2}$. The formula for the 1-loop scalar potential (4.7) applied to this case produces (dropping the dilaton dependence since $S$ is fixed at tree level)

$$
\delta V_{\left(g_{s}\right), 1-\text { loop }}^{K K}=\left(0 \cdot \frac{-3 \mathcal{C}^{K K}}{\mathcal{V}^{8 / 3}}+\frac{3 \alpha_{2}\left(\mathcal{C}^{K K}\right)^{2}}{\mathcal{V}^{10 / 3}}-\frac{6 \alpha_{3}\left(\mathcal{C}^{K K}\right)^{3}}{\mathcal{V}^{4}}+\mathcal{O}\left(\frac{1}{\mathcal{V}^{14 / 3}}\right)\right) W_{0}^{2} .
$$

To compare with (4.6) we recall that in supergravity the supertrace is proportional to the gravitino mass:

$$
\operatorname{STr}\left(M^{2}\right) \simeq m_{3 / 2}^{2}=e^{K} W_{0}^{2} \simeq \frac{1}{\mathcal{V}^{2}}
$$


The cut-off $\Lambda$ is identified with the compactification scale given by

$$
\Lambda=m_{K K} \simeq \frac{M_{s}}{R}=\frac{M_{s}}{\tau^{1 / 4}}=\frac{1}{\tau^{1 / 4}} \frac{M_{P}}{\sqrt{\mathcal{V}}}=\frac{M_{P}}{\mathcal{V}^{2 / 3}}
$$

Therefore in units of the Planck mass, (4.6) scales, in agreement with (4.8), as

$$
\delta V_{1-\text { loop }} \simeq 0 \cdot \frac{1}{\mathcal{V}^{8 / 3}}+\frac{1}{\mathcal{V}^{10 / 3}}+\frac{1}{\mathcal{V}^{4}} .
$$

We now use these results for the study of Kähler moduli stabilisation.

\subsection{LARGE Volume and String Loop Corrections}

The results reviewed in the previous section are very important for Kähler moduli stabilisation. The general picture for LVS which we presented in the previous sections, was neglecting the effect of string loop corrections to the scalar potential. However just looking at the Kähler potential we have seen that, in terms of powers of the Kähler moduli, the leading order $\alpha^{\prime}$ correction (2.1) scales as $\delta K_{\left(\alpha^{\prime}\right)} \sim \frac{1}{\mathcal{V}}$, whereas from (4.4), the scaling

behaviour of the Kaluza-Klein loop correction is $\delta K_{\left(g_{s}\right)}^{K K} \sim \frac{\sqrt{\tau}}{\mathcal{V}}$. Naively it seems incorrect to neglect $\delta K_{\left(g_{s}\right)}^{K K}$ while including the effects of $\delta K_{\left(\alpha^{\prime}\right)}$. However, as discussed in 10, 13, 12, due to the extended no-scale structure at the level of the scalar potential the $\alpha^{\prime}$ corrections dominate over the $g_{s}$ corrections. This allows loop corrections to be neglected compared to $\alpha^{\prime}$ corrections for the stabilisation of the volume.

However in our general analysis earlier, we saw that for fibration models the inclusion of $\alpha^{\prime}$ corrections still left almost flat directions corresponding to non blow-up moduli orthogonal to the overall volume. Loop corrections to the scalar potential are much more important than non-perturbative superpotential corrections, and we realise that they can play a crucial role in stabilising these non blow-up moduli transverse to the overall volume.

Thus we conclude that the extended no-scale structure renders the LVS robust not only because it allows $\delta V_{\left(g_{s}\right)}$ to be neglected when stabilising the volume, but also because it ensures that when $\delta V_{\left(g_{s}\right)}$ is introduced to lift the remaining flat directions, even though it will reintroduce a dependence in $V$ on $\mathcal{V}$ and blow-up moduli, it will not destroy the minimum already found but will give just a small perturbation around it.

The general picture is that all corrections - $\alpha^{\prime}$, loop and non-perturbative - play a rôle in a generic Calabi-Yau compactification. We can summarise our general analysis for the LVS as:

1. In order to stabilise all the Kähler moduli at exponentially large volume one needs at least one 4-cycle which is a blow-up mode resolving point-like singularities.

2. This 4-cycle, together with other blow-up modes possibly present, are fixed small by the interplay of non-perturbative and $\alpha^{\prime}$ corrections, which stabilise also the overall volume mode.

3. The $g_{s}$ corrections are subleading and so can be safely neglected. 
4. All the other 4-cycles, as those corresponding to fibrations, even though they have non-perturbative effects, cannot be stabilised small. Thus they are sent large so making their non-perturbative corrections negligible.

5. These moduli, which are large and transverse to the overall volume, can then be frozen by $g_{s}$ corrections, which dominate over the (tiny) non-perturbative ones.

In general $\delta V_{\left(g_{s}\right)}$ only lifts the flat directions associated to non blow-up moduli transverse to the overall volume. One could wonder whether they indeed yield a real minimum for such moduli as opposed to a runaway direction. We do not address this problem in general terms here and so in principle this looks like a model-dependent issue. However, as the overall volume is stabilised, the internal moduli space is compact. Therefore these non blow-up moduli cannot run-away to infinity and so we expect that loop corrections will induce a minimum for the potential. In fact, one example in the next section will illustrate this idea explicitly.

\section{Moduli Stabilisation via String Loop Corrections}

We will now see in detail how the inclusion of string loop corrections can affect the results found in the previous examples which, neglecting $g_{s}$ corrections, can be summarised as:

1. $\mathbb{C} P_{[1,1,1,6,9]}^{4} \rightarrow$ LVS without flat directions.

2. 3-parameter K3 Fibration with $\tau_{1}$ "small" and $a_{1} \tau_{1}>a_{3}\left\langle\tau_{3}\right\rangle \rightarrow$ LVS with an almost flat direction.

3. 3-parameter K3 Fibration with $\tau_{3} \ll \tau_{1}<\tau_{2} \rightarrow$ LVS with one flat direction.

4. $\mathbb{C} P_{[1,3,3,3,5]}^{4} \rightarrow$ LVS with a tachyonic direction.

5. $\mathbb{C} P_{[1,1,2,2,6]}^{4}$ and 3-parameter K3 Fibration with $\tau_{1}$ "small" and $a_{1} \tau_{1}<a_{3} \tau_{3} \rightarrow$ No LVS.

We shall find that the inclusion of loop corrections modifies the previous picture as follows:

1. $\mathbb{C} P_{[1,1,1,6,9]}^{4} \rightarrow$ Not affected by $\delta V_{\left(g_{s}\right)}$.

2. 3-parameter K3 Fibration with $\tau_{1}$ "small" and $a_{1} \tau_{1}>a_{3}\left\langle\tau_{3}\right\rangle \rightarrow \delta V_{\left(g_{s}\right)}$ ruins the almost flat direction $\Longrightarrow$ No LVS ${ }^{2}$.

3. 3-parameter K3 Fibration with $\tau_{3} \ll \tau_{1}<\tau_{2} \rightarrow \delta V_{\left(g_{s}\right)}$ lifts the flat direction $\Longrightarrow$ LVS without flat directions.

4. $\mathbb{C} P_{[1,3,3,3,5]}^{4} \rightarrow \delta V_{\left(g_{s}\right)}$ stabilises the tachyonic direction $\Longrightarrow$ LVS without flat directions.

\footnotetext{
${ }^{2}$ Notice that this case is the same as case 3 below but in a different region of moduli space. The fact that there is no LVS realised here only means that for this model the LARGE Volume is realised as in the conditions of case 3 but not in those for case 2. In particular it requires both fibration moduli to be large.
} 
5. $\mathbb{C} P_{[1,1,2,2,6]}^{4}$ and 3-parameter K3 Fibration with $\tau_{1}$ "small" and $a_{1} \tau_{1}<a_{3} \tau_{3} \rightarrow$ Not affected by $\delta V_{\left(g_{s}\right)}-$ still no LVS.

The $\mathbb{C} P_{[1,1,2,2,6]}^{4}$ case can never give large volume due to the fibration 4-cycle $\tau_{1}$ which is impossible to stabilise small. However in the example of the 3-parameter K3 fibration, LARGE Volume can be achieved by including a third Kähler modulus which is a local blow-up and then sending $\tau_{1}$ large. We shall use the expression (4.7) for the form of string loop corrections to the scalar potential.

\subsection{The single-hole Swiss cheese: $\mathbb{C} P_{[1,1,1,6,9]}^{4}$}

The influence of the $g_{s}$ corrections in the $\mathbb{C} P_{[1,1,1,6,9]}^{4}$ case has been studied in detail in [13]. The authors showed that the loop corrections are subleading and so can be neglected, as we claimed above. The loop corrected Kähler potential looks like

$$
\begin{aligned}
K & =K_{\text {tree }}+\delta K_{\left(\alpha^{\prime}\right)}+\delta K_{\left(g_{s}, \tau_{5}\right)}^{K K}+\delta K_{\left(g_{s}, \tau_{4}\right)}^{K K} \\
& =-2 \ln \mathcal{V}-\frac{\xi}{\mathcal{V} g_{s}^{3 / 2}}+\frac{g_{s} C_{5}^{K K} \sqrt{\tau_{5}}}{\mathcal{V}}+\frac{g_{s} C_{4}^{K K} \sqrt{\tau_{4}}}{\mathcal{V}}
\end{aligned}
$$

but due to the "extended no scale structure", we obtain for the scalar potential

$$
\begin{aligned}
V & =V_{n p}+V_{\left(\alpha^{\prime}\right)}+V_{\left(g_{s}, \tau_{5}\right)}^{K K}+V_{\left(g_{s}, \tau_{4}\right)}^{K K} \\
& =\frac{\lambda_{1} \sqrt{\tau_{4}} e^{-2 a_{4} \tau_{4}}}{\mathcal{V}}-\frac{\lambda_{2} W_{0} \tau_{4} e^{-a_{4} \tau_{4}}}{\mathcal{V}^{2}}+\frac{3 \xi W_{0}^{2}}{4 \mathcal{V}^{3} g_{s}^{3 / 2}}+\frac{g_{s}^{2}\left(C_{5}^{K K}\right)^{2}}{\mathcal{V}^{3} \sqrt{\tau_{5}}}+\frac{g_{s}^{2}\left(C_{4}^{K K}\right)^{2}}{\mathcal{V}^{3} \sqrt{\tau_{4}}}
\end{aligned}
$$

Without taking the loop corrections into account, we have found a minimum located at $\mathcal{V} \sim e^{a_{4} \tau_{4}} \Leftrightarrow a_{4} \tau_{4} \sim \ln \mathcal{V}$. Therefore the various terms in (5.2) scale as

$$
\begin{aligned}
V & =V_{n p}+V_{\left(\alpha^{\prime}\right)}+V_{\left(g_{s}, \tau_{5}\right)}^{K K}+V_{\left(g_{s}, \tau_{4}\right)}^{K K} \\
& \sim \frac{\sqrt{\ln \mathcal{V}}}{\mathcal{V}^{3}}-\frac{\ln \mathcal{V}}{\mathcal{V}^{3}}+\frac{1}{\mathcal{V}^{3}}+\frac{1}{\mathcal{V}^{10 / 3}}+\frac{1}{\mathcal{V}^{3} \sqrt{\ln \mathcal{V}}}
\end{aligned}
$$

and it is straightforward to realise that at exponentially large volume the last two terms in (5.3) are suppressed with respect to the first three ones.

\subsection{The multiple-hole Swiss cheese: $\mathbb{C} P_{[1,3,3,3,5]}^{4}$}

In Section 3.2 we have seen that if the non-perturbative corrections in the SM cycle $\tau_{S M}$ are absent, the F-term scalar potential (3.12) for the $\mathbb{C} P_{[1,3,3,3,5]}^{4}$ Calabi-Yau does not present a LVS with all the Kähler moduli stabilised. Following the same procedure as in [19], we shall now illustrate how the $g_{s}$ corrections can turn the maximum in the $\tau_{S M}$ direction into a minimum without destroying the exponentially large volume minimum $\mathcal{V} \sim \sqrt{\tau_{E 3}} e^{2 \pi \tau_{E 3}}$.

To derive the conjectured scaling behaviour of the loop corrections, we use the formula (4.7) setting $\mathcal{C}_{i}^{K K}=\operatorname{Re}(S) \forall i$ and $W_{0}=1$. Two stacks of D7-branes wrap the $\tau_{S M}$ and $\tau_{c}$ cycle respectively and both will give rise to Kaluza-Klein $g_{s}$ corrections. From (4.7), we 
estimate the first kind of corrections by writing the overall volume (3.10) in the $\left(\tau_{a}, \tau_{S M}, \tau_{c}\right)$ basis and computing the relevant elements of the direct Kähler metric. We find:

$$
\mathcal{V}=\sqrt{\frac{2}{45}}\left(\tau_{a}^{3 / 2}-\frac{1}{3}\left(3 \tau_{S M}+2 \tau_{c}\right)^{3 / 2}-\frac{\sqrt{5}}{3} \tau_{c}^{3 / 2}\right)
$$

along with

$$
\frac{\partial^{2} K_{\text {tree }}}{\partial \tau_{S M}^{2}} \simeq \frac{3}{\sqrt{10}} \frac{1}{\mathcal{V} \sqrt{3 \tau_{S M}+2 \tau_{c}}}
$$

and

$$
\frac{\partial^{2} K_{\text {tree }}}{\partial \tau_{c}^{2}} \simeq \frac{2 \sqrt{2}}{3 \sqrt{5}}\left(\frac{\sqrt{5}}{4 \sqrt{\tau_{c}}}+\frac{1}{\sqrt{3 \tau_{S M}+2 \tau_{c}}}\right) \frac{1}{\mathcal{V}}
$$

where in the large volume limit we have approximated the volume as $\mathcal{V} \simeq \sqrt{\frac{2}{45}} \tau_{a}^{3 / 2}$. Thus the Kaluza-Klein loop corrections to (3.12) look like

$$
\delta V_{\left(g_{s}\right)}^{K K} \simeq\left(\frac{5}{\sqrt{\tau_{c}}}+\frac{13 \sqrt{5}}{\sqrt{3 \tau_{S M}+2 \tau_{c}}}\right) \frac{1}{15 \sqrt{2} \mathcal{V}^{3}} .
$$

Writing (5.7) back in terms of $\tau_{S M}$ and $\tau_{E 3}=\tau_{c}+\tau_{S M}$, we obtain

$$
\delta V_{\left(g_{s}\right)}^{K K} \simeq\left(\frac{5}{\sqrt{\tau_{E 3}-\tau_{S M}}}+\frac{13 \sqrt{5}}{\sqrt{2 \tau_{E 3}+\tau_{S M}}}\right) \frac{1}{15 \sqrt{2} \mathcal{V}^{3}} .
$$

Due to the particulary simple form of the volume (5.4), it is very sensible to expect that the winding corrections will scale like the Kaluza-Klein ones (5.8). Therefore adding (5.8) to $(3.12)$ we end up with

$$
\begin{aligned}
V+\delta V_{\left(g_{s}\right)}= & \frac{\lambda_{1}\left(\sqrt{5\left(2 \tau_{E 3}+\tau_{S M}\right)}+\sqrt{\tau_{E 3}-\tau_{S M}}\right) e^{-4 \pi \tau_{E 3}}}{\mathcal{V}}-\frac{3 \lambda_{2} \tau_{E 3} e^{-2 \pi \tau_{E 3}}}{\mathcal{V}^{2}} \\
& +\frac{\lambda_{3}}{\mathcal{V}^{3}}+\left(\frac{\lambda_{4}}{\sqrt{\tau_{E 3}-\tau_{S M}}}+\frac{\lambda_{5}}{\sqrt{2 \tau_{E 3}+\tau_{S M}}}\right) \frac{1}{\mathcal{V}^{3}} .
\end{aligned}
$$

We notice that the string loop corrections are suppressed with respect to the $\alpha^{\prime}$ ones by a factor of $1 / \sqrt{\tau_{E 3}}$ and so do not affect the large volume minimum $\mathcal{V} \sim \sqrt{\tau_{E 3}} e^{2 \pi \tau_{E 3}}$ given that we require $\tau_{E 3} \gg 1$ to neglect higher order instanton contributions. On the contrary $\delta V_{\left(g_{s}\right)}$ can become important to fix the SM direction when $\tau_{S M}$ gets small. In fact, the maximum in that direction is now accompanied by a minimum, as illustrated in Figure 3.

Thus we have shown that $g_{s}$ corrections can indeed freeze the SM direction so giving rise to a LVS without any tachyonic direction. The physics of this stabilisation is simply that if a D7-brane wraps a 4-cycle, then loop corrections induced by the brane will become large as the cycle size collapses. This repels the modulus from collapsing and induces a minimum of the the potential.

This example is illustrative in nature and shows how a cycle, which is required to be small and which does not admit nonperturbative effects, can potentially be stabilised by 


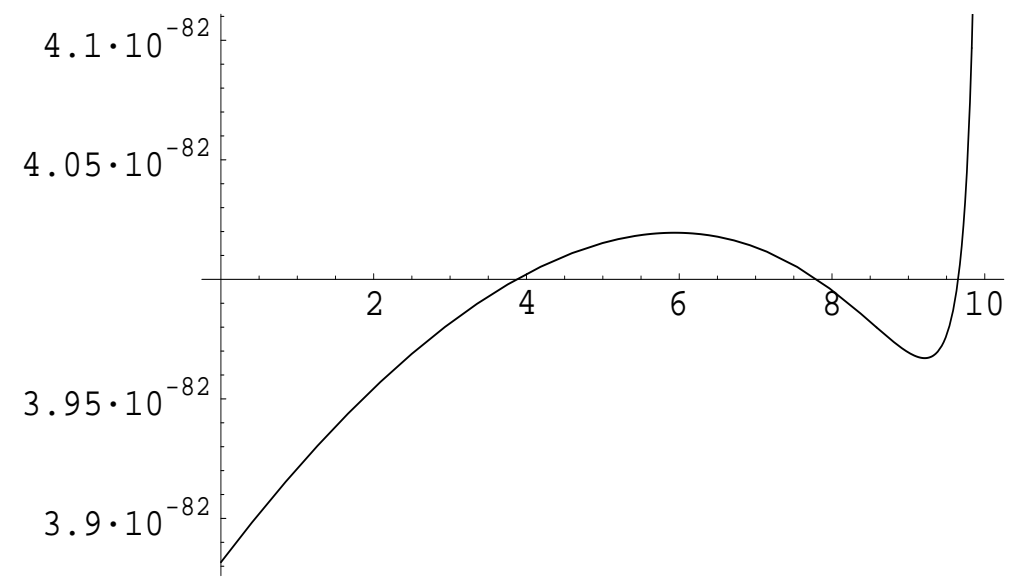

Figure 3: $\tau_{S M}$ fixed by string loop corrections. The numerical values used are $\lambda_{1}=\lambda_{2}=1$, $\lambda_{3}=50, \lambda_{4}=\lambda_{5}=5$ and then we have fixed $\tau_{E 3}=10$ and $\mathcal{V}=\sqrt{10} e^{20 \pi}$.

loop corrections. In a fully realistic model, the D-term contribution to the potential should also be included and the combined F- and D-term potential studied. Usually the D-term will include, besides the Fayet-Iliopoulos term depending on the moduli, also the charged matter fields. Minimising the D-term will generically fix one of the Standard Model singlets to essentially cancel the Fayet-Iliopoulos term. Thus we can foresee a scenario in which the Standard Model cycle is fixed by string loop corrections whereas the D-term fixes not the size of that cycle but instead the VEV of a Standard Model singlet as a function of the moduli. In this way we address the challenge of [14. The form of the D-term however depends on the model and in particular on the details of the charged matter content and whether or not they acquire VEVs. We therefore do not try and specify this, but note that it will be necessary to include it in a realistic model.

\subsection{2-Parameter K3 Fibration: $\mathbb{C} P_{[1,1,2,2,6]}^{4}$}

One could wonder whether including the string loop corrections in the case of the K3 Fibration with two Kähler moduli treated in Section 3.3, could generate an exponentially large volume minimum which was absent when only non-perturbative and the $\alpha^{\prime}$ corrections are included. In reality, the answer is negative as these further perturbative corrections produce a contribution $V_{\left(g_{s}\right)}^{K K}+V_{\left(g_{s}\right)}^{W}$ to the scalar potential (3.21), which is subdominant and cannot help to stabilise the moduli. In fact, in the large volume limit (3.17) and for 
$W_{0} \sim \mathcal{O}(1)$, the full corrected scalar potential, now takes the form

$$
\begin{aligned}
V= & V_{n p}+V_{(\alpha \prime)}+V_{\left(g_{s}, \tau_{1}\right)}^{K K}+V_{\left(g_{s}, \tau_{2}\right)}^{K K}+V_{\left(g_{s}, \tau_{1}\right)}^{W}+V_{\left(g_{s}, \tau_{2}\right)}^{W} \simeq \\
\simeq & -\frac{4}{\mathcal{V}^{2}} W_{0} a_{1} \tau_{1} e^{-a_{1} \tau_{1}}+\frac{3}{4} \frac{\xi \operatorname{Re}(S)^{3 / 2}}{\mathcal{V}^{3}} W_{0}^{2} \\
& +\frac{W_{0}^{2}}{\mathcal{V}^{2}}\left(\frac{\left(\mathcal{C}_{1}^{K K}\right)^{2}}{\operatorname{Re}(S)^{2}} \frac{1}{\tau_{1}^{2}}+\frac{\left(\mathcal{C}_{2}^{K K}\right)^{2}}{\operatorname{Re}(S)^{2}} \frac{1}{2} \frac{\tau_{1}}{\mathcal{V}^{2}}-2 \mathcal{C}_{1}^{W} \frac{\tau_{1}}{\mathcal{V}^{2}}-\frac{2 \mathcal{C}_{2}^{W}}{\mathcal{V} \sqrt{\tau_{1}}}\right) \\
\simeq & -\frac{4}{\mathcal{V}^{2}} W_{0} a_{1} \tau_{1} e^{-a_{1} \tau_{1}}+\frac{3}{4} \frac{\xi \operatorname{Re}(S)^{3 / 2}}{\mathcal{V}^{3}} W_{0}^{2}+\frac{W_{0}^{2}}{\mathcal{V}^{2}}\left(\frac{\left(\mathcal{C}_{1}^{K K}\right)^{2}}{\operatorname{Re}(S)^{2}} \frac{1}{\tau_{1}^{2}}-\frac{2 \mathcal{C}_{2}^{W}}{\mathcal{V} \sqrt{\tau_{1}}}\right) .
\end{aligned}
$$

First of all we have to check that the minimum in the volume is exponentially large. Therefore let us take the derivative

$$
\frac{4 \mathcal{V}^{4}}{W_{0}^{2}} \frac{\partial V}{\partial \mathcal{V}}=\left(\frac{32}{W_{0}} a_{1} \tau_{1} e^{-a_{1} \tau_{1}}-\frac{\left(\mathcal{C}_{1}^{K K}\right)^{2}}{\operatorname{Re}(S)^{2}} \frac{8}{\tau_{1}^{2}}\right) \mathcal{V}+\left(\frac{24 \mathcal{C}_{2}^{W}}{\sqrt{\tau_{1}}}-9 \xi \operatorname{Re}(S)^{3 / 2}\right)=0
$$

whose solution is

$$
\langle\mathcal{V}\rangle=\frac{3}{8} \frac{\operatorname{Re}(S)^{2}\left\langle\tau_{1}\right\rangle^{3 / 2} W_{0}\left(8 \mathcal{C}_{2}^{W}-3 \sqrt{\left\langle\tau_{1}\right\rangle} \xi \operatorname{Re}(S)^{3 / 2}\right)}{\left(\left(\mathcal{C}_{1}^{K K}\right)^{2} W_{0} e^{a_{1}\left\langle\tau_{1}\right\rangle}-4 a_{1} \operatorname{Re}(S)^{2}\left\langle\tau_{1}\right\rangle^{3}\right)} e^{a_{1}\left\langle\tau_{1}\right\rangle} .
$$

From $(5.12)$ we realise that in order to have an exponentially large volume, we need to fine tune $\left(\mathcal{C}_{1}^{K K}\right)^{2} \sim e^{-a_{1} \tau_{1}} \ll 1$. We assume that this is possible and so the denominator of (5.12) scales as

$$
W_{0}-4 a_{1} \operatorname{Re}(S)^{2}\left\langle\tau_{1}\right\rangle^{3} \simeq-4 a_{1} \operatorname{Re}(S)^{2}\left\langle\tau_{1}\right\rangle^{3},
$$

given that we are working in a regime where $W_{0} \sim \mathcal{O}(1), \operatorname{Re}(S) \simeq 10$ and $a_{1} \tau_{1} \gg 1$. Finally the VEV of the volume reads

$$
\langle\mathcal{V}\rangle \simeq \frac{3}{8} \frac{W_{0}\left(8 \mathcal{C}_{2}^{W}-3 \sqrt{\left\langle\tau_{1}\right\rangle} \xi \operatorname{Re}(S)^{3 / 2}\right)}{-4 a_{1}\left\langle\tau_{1}\right\rangle^{3 / 2}} e^{a_{1}\left\langle\tau_{1}\right\rangle},
$$

with $\mathcal{C}_{2}^{W}$ chosen such that

$$
\left(1-\frac{3 \sqrt{\left\langle\tau_{1}\right\rangle} \xi \operatorname{Re}(S)^{3 / 2}}{8 \mathcal{C}_{2}^{W}}\right)<0
$$

to have a positive result. Now we neglect the $\left(\mathcal{C}_{1}^{K K}\right)^{2}$ term in $V(5.10)$ when we perform the derivative with respect to $\tau_{1}$ and we obtain

$$
\frac{\mathcal{V}^{2}}{W_{0}} \frac{\partial V}{\partial \tau_{1}}=4 a_{1} e^{-a_{1}\left\langle\tau_{1}\right\rangle}\left(a_{1}\left\langle\tau_{1}\right\rangle-1\right)+\frac{W_{0} \mathcal{C}_{2}^{W}}{\langle\mathcal{V}\rangle\left\langle\tau_{1}\right\rangle^{3 / 2}}=0
$$

Substituting back (5.14), (5.16) becomes

$$
a_{1}\left\langle\tau_{1}\right\rangle=1+\frac{1}{3\left(1-\frac{3 \sqrt{\left\langle\tau_{1}\right\rangle} \xi \operatorname{Re}(S)^{3 / 2}}{8 \mathcal{C}_{2}^{W}}\right)},
$$


but (5.15) forces us to get $a_{1}\left\langle\tau_{1}\right\rangle<1$, clearly in disagreement with our starting point when we ignored higher order instanton corrections. Hence we conclude that the inclusion of the string loop corrections does not help to stabilise the moduli at exponentially large volume since they render this attempt even worse.

\subsection{3-Parameter K3 Fibration}

The results of the study of the K3 Fibration with three Kähler moduli are summarised in the table at the end of Section 3.4.1. Let us consider now how these interesting cases are modified by the inclusion of the string loop corrections. Without loss of generality, we set $\gamma=1$ and $\xi=2$. Moreover, we choose $\alpha=1 / 2$ and $\beta=2 / 3$ so that when the large volume limit is taken sending $\tau_{1} \rightarrow \infty$, the internal volume (3.28) takes the same form of the volume (3.14) for the $\mathbb{C} P_{[1,1,2,2,6]}^{4}$ case:

$$
\mathcal{V} \simeq \frac{1}{2} \sqrt{\tau_{1}}\left(\tau_{2}-\frac{2}{3} \tau_{1}\right)
$$

We shall work in the regime $W_{0}=1$ where the perturbative corrections are important. We shall now study the $g_{s}$ corrections to the scalar potential in $\tau_{1}$ and $\tau_{2}$ both in the $\tau_{1} \rightarrow \infty$ and " $\tau_{1}$ small" case.

Before doing that, we notice that the loop correction to $V$ in $\tau_{3}$ does not depend on the particular way we treat $\tau_{1}$ in the large volume limit and is subdominant to the one in $\alpha^{\prime}$. Thus we will confidently neglect it. More precisely, it could modify the exact locus of the minimum but not the main feature of the model, that is the presence of an exponentially large volume. In fact, given that the form of the "33" element of the Kähler matrix (3.35) is

$$
K_{33}^{0}=\frac{3}{4 \mathcal{V} \sqrt{\tau_{3}}},
$$

in analogy with (5.2), the 1-loop correction to the scalar potential in $\tau_{3}$ (4.7) takes the form

$$
\delta V_{\left(g_{s}\right), \tau_{3}}=\frac{\left(g_{s}^{2}\left(\mathcal{C}_{3}^{K K}\right)^{2}+2 \mathcal{C}_{3}^{W}\right)}{\sqrt{\tau_{3}} \mathcal{V}^{3}},
$$

which is subdominant compared with the $\alpha^{\prime}$ corrections and so is negligible. We will now try to address the problem left unsolved in the previous section. Without loop corrections it was possible to find an exponentially large volume in this class of models but there was still a flat direction left, which we named $\Omega$. Let us see now how this direction is lifted.

Let us first work in the region: $\tau_{3} \ll \tau_{1}<\tau_{2}$. We recall the form of the scalar potential and the Kähler potential without loop corrections:

$$
\begin{gathered}
V=\frac{16 a_{3}^{2}}{3 \mathcal{V}} \sqrt{\tau_{3}} e^{-2 a_{3} \tau_{3}}-\frac{4}{\mathcal{V}^{2}} a_{3} \tau_{3} e^{-a_{3} \tau_{3}}+\frac{3}{2 g_{s}^{3 / 2} \mathcal{V}^{3}}, \\
K=K_{\text {tree }}+\delta K_{\left(\alpha^{\prime}\right)} \underset{\mathcal{V} \gg 1}{\simeq}-2 \ln \mathcal{V}-\frac{2}{g_{s}^{3 / 2} \mathcal{V}} .
\end{gathered}
$$


We study now the possible corrections to $V$ coming from $\tau_{1}$ and $\tau_{2}$ according to the general 1-loop formula (4.7). The string loop corrections in $\tau_{1}$ and $\tau_{2}$ take the general form:

$$
\delta V_{\left(g_{s}\right), \tau_{1}}+\delta V_{\left(g_{s}\right), \tau_{2}}=\frac{A}{\tau_{1}^{2} \mathcal{V}^{2}}+\frac{B \sqrt{\tau_{1}}}{\left(\tau_{2}-2 \tau_{1}\right) \mathcal{V}^{3}}+\frac{C}{\sqrt{\tau_{1}} \mathcal{V}^{3}}+\frac{D \tau_{1}}{\mathcal{V}^{4}}
$$

where the first two terms come from the loop corrections on $\tau_{1}$, the third from those of $\tau_{2}$ and the last one from both. Here $A, D>0$. In fact, due to the "extended no-scale structure" which causes the vanishing of the leading Kaluza-Klein correction to $V$, we know the sign of the coefficient of $\delta V_{\left(g_{s}\right)}^{K K}$ because the parameter is squared (see (4.7)). For generic values of $A, B, C, D$ we expect to lift the flat direction $\Omega$. The relevant cases to consider correspond to either having branes wrapping only the $\tau_{1}$ or $\tau_{2}$ cycle, which would set $B=0$ or $A=C=0$ respectively and the relative signs and magnitude of $B$ and $C$ (this corresponds to choosing the values of $C_{1,2}^{W}$ ).

It is convenient to consider the potential in terms of $\mathcal{V}$ and $\Omega$ instead of $\tau_{1}$ and $\tau_{2}$. Inverting the relation (3.49) for $\alpha=1 / 2$ and $\beta=2 / 3$, we get

$$
\tau_{1}=\left(\frac{3(\Omega-\mathcal{V})}{2}\right)^{2 / 3}, \quad \tau_{2}=\left(\frac{2}{3(\Omega-\mathcal{V})}\right)^{1 / 3}(\Omega+\mathcal{V})
$$

Substituting these results back in (5.23) we can find the relevant dependence of the scalar potential on $\Omega$. Then consistency requirements imply that any meaningful minimum must lie within the Kähler cone and in particular for $t_{1}>0, t_{2}>0$ and $\mathcal{V}>0$. In this case we have $\mathcal{V}=t_{1} t_{2}^{2}+\frac{2}{3} t_{2}^{3}$, and so the volume is automatically positive if both $t_{1}$ and $t_{2}$ is positive. The 2-cycle moduli, in terms of $\mathcal{V}$ and $\Omega$, read

$$
t_{1}=\left(\frac{2}{3}\right)^{2 / 3} \frac{(2 \mathcal{V}-\Omega)}{(\Omega-\mathcal{V})^{2 / 3}}, \quad t_{2}=\left(\frac{3}{2}\right)^{1 / 3}(\Omega-\mathcal{V})^{1 / 3}
$$

Therefore the Kähler cone in terms of $\mathcal{V}$ and $\Omega$ is given by $\mathcal{V}<\Omega<2 \mathcal{V}$ and the minimum has to lie in this region. In fact, when $\Omega \rightarrow \mathcal{V}^{+}, t_{1} \rightarrow \infty$ and $t_{2} \rightarrow 0$, whereas when $\Omega \rightarrow(2 \mathcal{V})^{-}$, $t_{1} \rightarrow 0$ while $t_{2} \rightarrow\left(\frac{3 \mathcal{V}}{2}\right)^{1 / 3}$. With our choice of parameters, (3.48) yields $\langle\mathcal{V}\rangle \sim 2 \cdot 10^{21}$. Thus the two walls of the Kähler cone lie at $\langle\mathcal{V}\rangle \sim 2 \cdot 10^{21}$ and $2\langle\mathcal{V}\rangle \sim 4 \cdot 10^{21}$.

Setting $A=1, B=40, C=20$ and $D=13 / 18$ the scalar potential (5.23) becomes

$$
\delta V=\frac{13 \Omega^{3}-172 \Omega^{2} \mathcal{V}+73 \Omega \mathcal{V}^{2}+78 \mathcal{V}^{3}}{2^{2 / 3} 3^{1 / 3} 6(\Omega-2 \mathcal{V})(\Omega-\mathcal{V})^{4 / 3} \mathcal{V}^{4}}
$$

and admits a minimum in the region $\mathcal{V}<\Omega<2 \mathcal{V}$ located at $\langle\Omega\rangle \sim 2.5 \cdot 10^{21}$ as shown in Figure 4. The physics is simply that the moduli are repelled from the two walls of the Kähler cone and must therefore be stabilised inbetween as the space is compact.

A completely different potential profile appears if we do not have branes wrapping one of the cycles. For instance, for no branes wrapping the $\tau_{2}$ cycle, the potential (5.23) now becomes (with $A=1, B=0, C=-20, D=2 / 9$ )

$$
\delta V=\left(\frac{2}{3}\right)^{1 / 3} \frac{\Omega^{2}-62 \Omega \mathcal{V}+63 \mathcal{V}^{2}}{3(\Omega-\mathcal{V})^{4 / 3} \mathcal{V}^{4}} .
$$




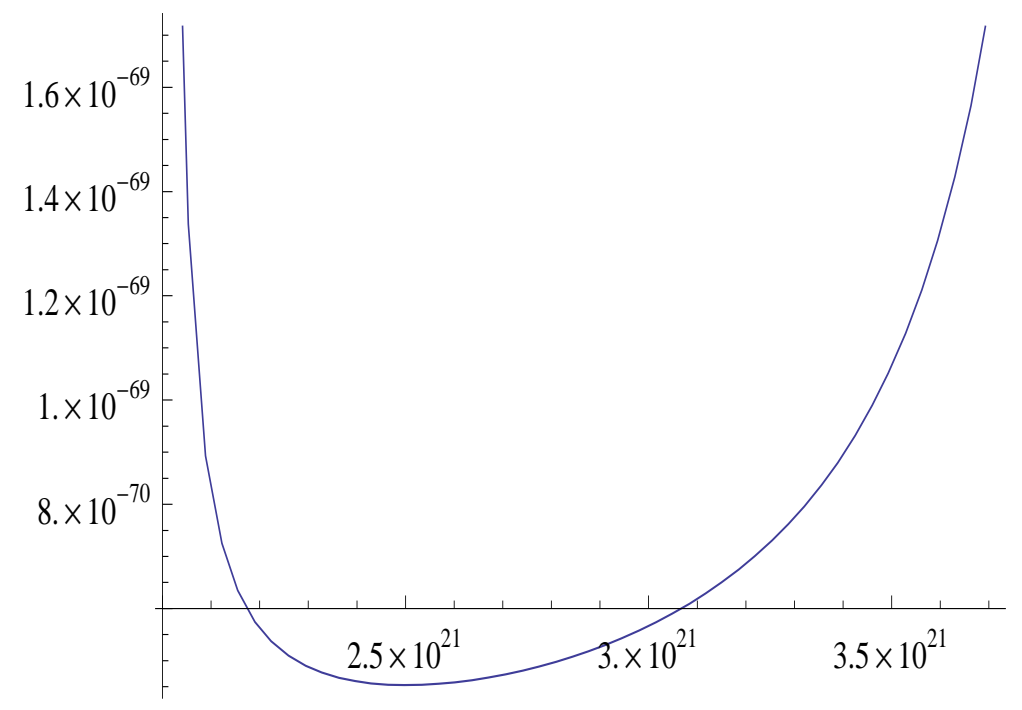

Figure 4: $V$ versus $\Omega$ at $\mathcal{V}$ and $\tau_{3}$ fixed.

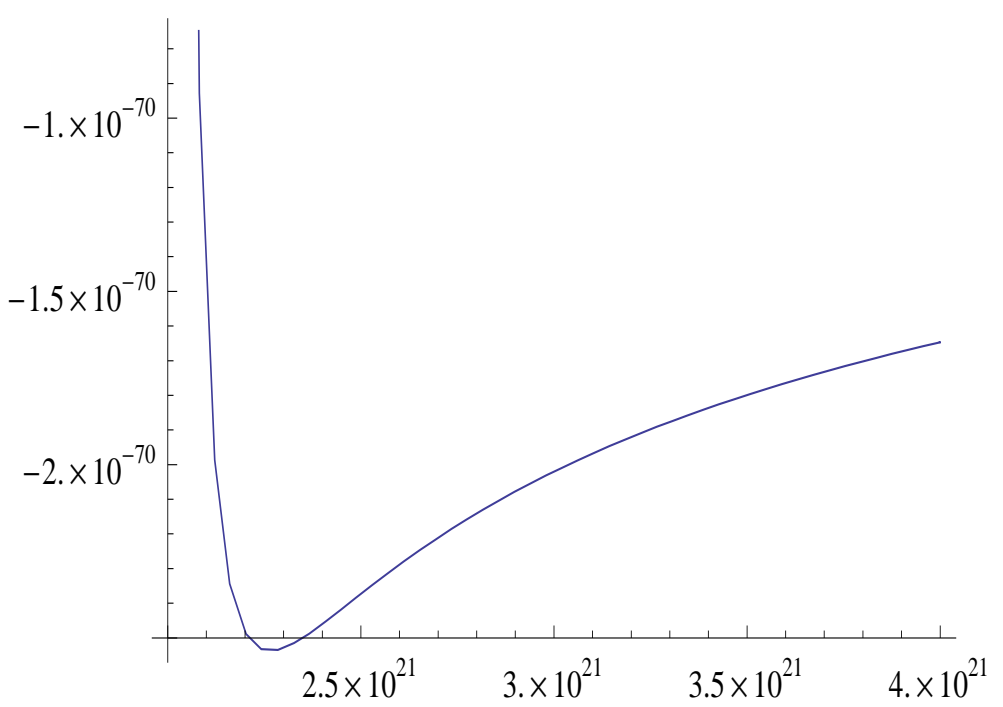

Figure 5: No branes wrapping the $\tau_{2}$ cycle with $\mathcal{C}_{2}^{W}=10: V$ versus $\Omega$ at $\mathcal{V}$ and $\tau_{3}$ fixed.

Also this case produces an interesting minimum shown in Figure 5 and located correctly in the region $\mathcal{V}<\Omega<2 \mathcal{V}$ at $\langle\Omega\rangle \sim 2.3 \cdot 10^{21}$.

Considering the matrix of second derivatives in the $\left(\tau_{1}, \tau_{2}\right)$ space $M_{i j}=V_{i j}$ and using the known expression for the Kähler metric $K_{i j}$ we construct the matrix $K^{-1} M$. The two eigenvalues of this matrix correspond to the mass-squared of the canonically normalised particles and look like

$$
m^{2} \sim 1 / \mathcal{V}^{3}, 1 / \mathcal{V}^{10 / 3}
$$

The first one corresponds to the standard volume modulus as in the Swiss cheese examples and the second one to the originally flat direction $\Omega$. 
Finally we have looked at minima in which $\tau_{2} \gg \tau_{1}$. This would be an interesting configuration because of the following observation: since $t_{2}=\sqrt{\tau_{1}}$ and $t_{1}=\left(\tau_{2}-2 \tau_{1}\right) / 2 \sqrt{\tau_{1}}$, we can see that $\tau_{2} \gg \tau_{1}$ would imply that $t_{1} \gg t_{2}$ and we would effectively have a very anisotropic compactification with the 2-cycle much bigger than its dual 4-cycle. This could then lead to a realisation of the supersymmetric 2 large extra dimensions scenario 17, 18] in which the extra dimension could be as large as a fraction of a millimetre. This would correspond to looking for solutions $\langle\Omega\rangle=(1+\epsilon)\langle\mathcal{V}\rangle$ with $\epsilon \rightarrow 0$.

However, we have shown that in the case $\tau_{1}$ "small", the inclusion of the string loop corrections ruins the presence of the almost flat direction which we had found before, without being able to produce an actual minimum at exponentially large volume with all moduli stabilised in a reliable region of moduli space.

\section{Conclusions and Potential Applications}

This paper has studied the general conditions needed to find exponentially large volume in type IIB compactifications. The necessary and sufficient conditions are simple to state: negative Euler number, more than one Kähler moduli with at least one of them being a blow-up mode resolving a point-like singularity.

We have also uncovered the important rôle played by $g_{s}$ corrections in moduli stabilisation. This has allowed us to find new classes of LVS with a fibration structure in which not only the volume but the fibre moduli are exponentially large whereas the blow-up modes are stabilised at the usual small values. Therefore in general all of $\alpha^{\prime}$, non-perturbative and $g_{s}$ corrections, may be important to stabilise the different classes of Kähler moduli.

Here we briefly discuss some of the applications. First, our results do not appear to change significantly the standard phenomenology of LVS explored in [26, 27], where we imagine the Standard Model localised on D7 branes wrapping a small 4-cycle. The reason is that the volume modulus is still the main source of supersymmetry breaking leading to an approximate no-scale structure, which can be argued in general terms [28]. As the Standard Model is localised around a blow-up cycle, the effects from other exponentially large moduli will be suppressed. However, it may be interesting to explore the potential implications of hidden sectors localised on those cycles. Also, in the multiple-hole Swiss cheese case where the Standard Model cycle is stabilised by perturbative rather than nonperturbative effects, the general structure of soft terms will not change significantly, again since the main source of supersymmetry breaking is the volume modulus, but it would be interesting to study this case in further detail.

A potentially more interesting application is to cosmology. The cosmological implications of LVS have been explored in [29, 30, 31, 32] only for Swiss cheese compactifications. Small moduli were found to be good candidates for inflatons as long as $h_{11}>2$ without the need to fine tune. However a difficulty with this is that loop corrections are expected to modify this result if there is a D7 brane wrapping the inflaton cycle, while if there is no such brane then it is difficult to reheat the Standard Model brane since there is no direct coupling of the inflaton to Standard Model fields. 
The volume modulus is not suitable for inflation as $m_{\mathcal{V}} \sim H$ and so it suffers directly from the $\eta$ problem. However for fibration models such as $\mathbb{C} P_{[1,1,2,2,6]}^{4}$, there is the transverse field $\Omega$ which is stabilised by the loop corrections. As the loop corrections are parametrically weaker than the $\alpha^{\prime}$ corrections which stabilise the volume, $\Omega$ is parametrically lighter than the volume modulus and thus the Hubble scale. In fact $m_{\Omega} \sim \mathcal{V}^{-(3 / 2+\alpha)}$, with $\alpha=1 / 6$. It follows that the slow-roll $\eta$ parameter is

$$
\eta \sim M_{P}^{2} \frac{m_{\Omega}^{2}}{H^{2}} \sim \frac{1}{\mathcal{V}^{1 / 3}} \ll 1
$$

Therefore such fibration models seem promising for string theory realisations of modular inflation, as at large volume the mass scale induced by loop corrections is parametrically smaller than the Hubble scale. A detailed study of the potential for large values of the field, away from the minimum, will be required in order to see if this is a viable model of inflation, including the value of density perturbations and the potential for reheating.

The fact that the spectrum of moduli fields includes further candidate light fields, besides the volume modulus, is a new source for the cosmological moduli problem. In the LARGE volume scenario this problem is already present as long as the string scale is smaller than $10^{13} \mathrm{GeV}$ since the volume modulus would be lighter than $10 \mathrm{TeV}$, and coupling with gravitational strength interactions it would overclose the universe or decay so late to ruin nucleosynthesis. Given a solution to this problem - such as a late period of inflation the corresponding modulus becomes a dark matter candidate. With an intermediate string scale and $\mathrm{TeV}$ supersymmetry, the volume modulus has a mass $m \sim 1 \mathrm{MeV}$. The additional light moduli fields are also potential dark matter candidates and have masses $m \sim 10 \mathrm{keV}$. Furthermore, they can decay into photons with a clean monochromatic line similar to the volume modulus. A proper analysis of their couplings to photons along the lines of [24] should be made in order to see if this effect could be eventually detected.

It is worth pointing that the multiple hole Swiss cheese example provides an explicit example of Kähler moduli inflation, in which at least three Kähler moduli were needed (but no explicit example was provided in [29]). Also this is a good example to explore the issue about stabilisation of the Standard Model cycle that has to be small (and then a blow-up mode) but without the presence of a non-perturbative superpotential which is not desired if the corresponding axion is the QCD axion [19] and if D-terms could induce a breaking of the Standard Model group [14]. Our results indicate that it is actually possible to achieve this.

We would finally like to emphasise that this is only a first attempt to investigate the relevance of loop corrections in the LVS and much work remains to be done. In particular, although we have used a well motivated volume dependence of the leading quantum corrections to the Kähler potential, explicit calculations are still lacking. While we believe that given the general importance of loop corrections, it is important to study their effects even with incomplete knowledge of their form, further information about these corrections for general Calabi-Yaus is very desirable.

\section{Acknowledgements}


We thank M. Berg, C.P. Burgess, P. Candelas, X. de la Ossa, F. Denef, M. Haack, M. Kreuzer, A. Maharana and L. McAllister for useful conversations. MC is partially funded by St John's College, EPSRC and CET. JC is funded by Trinity College, Cambridge and also thanks the University of Texas at Austin for hospitality while some of this work was carried out. FQ is partially funded by STFC and a Royal Society Wolfson merit award. He also thanks the Mitchell family for hospitality at the Cambridge-Texas A\& M Cook's Branch meeting 2008. This material is based upon work supported by the National Science Foundation under Grant No. PHY-0455649.

\section{A. Proof of the LARGE Volume Claim}

Let us now present a comprehensive argument in favour of the LARGE Volume Claim which establishes the existence of LARGE Volumes in IIB string compactifications.

\section{A.1 Proof for $N_{\text {small }}=1$}

Proof. (LARGE Volume Claim for $N_{\text {small }}=1$ ) Let us start from the scalar potential (2.9) which we now rewrite as

$$
V=V_{n p 1}+V_{n p 2}+V_{\left(\alpha^{\prime}\right)}
$$

and perform the large volume limit as described in (2.10) with $N_{\text {small }}=1$ corresponding to $\tau_{1}$. In this limit $V_{\left(\alpha^{\prime}\right)}$ behaves as

$$
V_{\left(\alpha^{\prime}\right)} \underset{\mathcal{V} \rightarrow \infty}{\longrightarrow}+\frac{3 \hat{\xi}}{4 \mathcal{V}^{3}} e^{K_{c s}}|W|^{2}+\mathcal{O}\left(\frac{1}{\mathcal{V}^{4}}\right) .
$$

We also point out that

$$
e^{K} \underset{\mathcal{V} \rightarrow \infty}{\longrightarrow} \frac{e^{K_{c s}}}{\mathcal{V}^{2}}+\mathcal{O}\left(\frac{1}{\mathcal{V}^{3}}\right)
$$

Let us now study $V_{n p 1}$ which reduces to

$$
V_{n p 1} \underset{\mathcal{V} \rightarrow \infty}{\longrightarrow} e^{K} K_{11}^{-1} a_{1}^{2}\left|A_{1}\right|^{2} e^{-a_{1}\left(T_{1}+\bar{T}_{1}\right)}=\frac{K_{11}^{-1}}{\mathcal{V}^{2}} a_{1}^{2}\left|A_{1}\right|^{2} e^{-2 a_{1} \tau_{1}}
$$

Switching to the study of $V_{n p 2}$, we find that

$$
V_{n p 2} \underset{\mathcal{V} \rightarrow \infty}{\longrightarrow}-e^{K} \sum_{k=1}^{h_{1,1}} K_{1 k}^{-1}\left[\left(a_{1} A_{1} e^{-a_{1} \tau_{1}} e^{-i a_{1} b_{1}} \bar{W} \partial_{\bar{T}_{k}} K\right)+\left(a_{1} \bar{A}_{1} e^{-a_{1} \tau_{1}} e^{+i a_{1} b_{1}} W \partial_{T_{k}} K\right)\right]
$$

where we have used the fact that $K_{1 k}^{-1}=K_{k 1}^{-1}$. Equation (A.5) can be rewritten as

$$
\begin{aligned}
& V_{n p 2} \underset{\mathcal{V} \rightarrow \infty}{\longrightarrow}-e^{K} \sum_{k=1}^{h_{1,1}} K_{1 k}^{-1}\left(\partial_{T_{k}} K\right) a_{1} e^{-a_{1} \tau_{1}}\left[\left(A_{1} \bar{W} e^{-i a_{1} b_{1}}\right)+\left(\bar{A}_{1} W e^{+i a_{1} b_{1}}\right)\right] \\
= & \left(X_{1} e^{+i a_{1} b_{1}}+\bar{X}_{1} e^{-i a_{1} b_{1}}\right),
\end{aligned}
$$

where

$$
X_{1} \equiv-e^{K} K_{1 k}^{-1}\left(\partial_{T_{k}} K\right) a_{1} \bar{A}_{1} W e^{-a_{1} \tau_{1}}
$$


We note that for a general Calabi-Yau, the following relation holds:

$$
K_{1 k}^{-1}\left(\partial_{T_{k}} K\right)=-2 \tau_{1}
$$

and thus the definition (A.7) can be simplified to

$$
X_{1} \equiv 2 e^{K} a_{1} \tau_{1}\left|A_{1}\right| e^{-i \vartheta_{1}}|W| e^{i \vartheta_{W}} e^{-a_{1} \tau_{1}}=\left|X_{1}\right| e^{i\left(\vartheta_{W}-\vartheta_{1}\right)} .
$$

Therefore

$$
V_{n p 2} \underset{\mathcal{V} \rightarrow \infty}{\longrightarrow}\left|X_{1}\right|\left(e^{+i\left(\vartheta_{W}-\vartheta_{1}+a_{1} b_{1}\right)}+e^{-i\left(\vartheta_{W}-\vartheta_{1}+a_{1} b_{1}\right)}\right)=2\left|X_{1}\right| \cos \left(\vartheta_{W}-\vartheta_{1}+a_{1} b_{1}\right) .
$$

$V_{n p 2}$ is a scalar function of the axion $b_{1}$ whereas $\vartheta_{1}$ and $\vartheta_{W}$ are to be considered just as parameters. In order to find a minimum for $V_{n p 2}$ let us set its first derivative to zero:

$$
\frac{\partial V_{n p 2}}{\partial b_{1}}=-2 a_{1}\left|X_{1}\right| \sin \left(\vartheta_{W}-\vartheta_{1}+a_{1} b_{1}\right)=0
$$

The solution of (A.11) is given by

$$
a_{1} b_{1}=p_{1} \pi+\vartheta_{1}-\vartheta_{W}, \quad p_{1} \in \mathbb{Z} .
$$

We have still to check the sign of the second derivative evaluated at $b_{1}$ as given in (A.12) and require it to be positive:

$$
\frac{\partial^{2} V_{n p 2}}{\partial b_{1}^{2}}=-2 a_{1}^{2}\left|X_{1}\right| \cos \left(\vartheta_{W}-\vartheta_{1}+a_{1} b_{1}\right)>0 \Longleftrightarrow p_{1} \in 2 \mathbb{Z}+1
$$

Thus we realise that at the minimum

$$
V_{n p 2}=-2\left|X_{1}\right|=-2|W|\left|A_{1}\right| a_{1} \tau_{1} \frac{e^{-a_{1} \tau_{1}}}{\mathcal{V}^{2}} .
$$

We notice that the phases of $W$ and $A_{1}$ do not enter into $V_{n p 2}$ once the axion has been properly minimised and so, without loss of generality, we can consider $W$ and $A_{1} \in \mathbb{R}^{+}$ from now on.

We may now study the full potential by combining equations (A.2), (A.4) and (A.14)

$$
V \simeq \frac{K_{11}^{-1}}{\mathcal{V}^{2}} A_{1}^{2} a_{1}^{2} e^{-2 a_{1} \tau_{1}}-\frac{W_{0}}{\mathcal{V}^{2}} A_{1} a_{1} \tau_{1} e^{-a_{1} \tau_{1}}+\frac{\hat{\xi}}{\mathcal{V}^{3}} W_{0}^{2},
$$

where we have substituted $W$ with its tree-level expectation value $W_{0}$ because the nonperturbative corrections are always subleading by a power of $\mathcal{V}$. Moreover, we have dropped all the factors since they are superfluous for our reasoning.

We would like to emphasize that we know that the first term in (A.15) is indeed positive. In fact it comes from

$$
K_{11}^{-1}\left(\partial_{1} W\right)\left(\partial_{1} \bar{W}\right)
$$

and we know that the Kähler matrix is positive definite since it gives rise to the kinetic terms. Moreover, as we have just seen, the second term in A.15) comes from the axion 
minimisation as so is definitely negative. Only the sign of $V_{\left(\alpha^{\prime}\right)}$ is in principle unknown, but the condition $h_{2,1}(X)>h_{1,1}(X)$ ensures that it is positive. This condition will turn out to be crucial in showing that the volume direction has indeed a minimum at exponentially large volume.

We need now to study the form of $K_{11}^{-1}$. For a general Calabi-Yau, the inverse Kähler matrix with $\alpha^{\prime}$ corrections included, reads [25]:

$$
K_{i j}^{-1}=-\frac{2}{9}(2 \mathcal{V}+\hat{\xi}) k_{i j k} t^{k}+\frac{4 \mathcal{V}-\hat{\xi}}{\mathcal{V}-\hat{\xi}} \tau_{i} \tau_{j}
$$

which at large volume becomes

$$
K_{i j}^{-1}=-\frac{4}{9} \mathcal{V} k_{i j k} t^{k}+4 \tau_{i} \tau_{j}+(\text { terms subleading in } \mathcal{V})
$$

Hence we can classify the behaviour of $K_{11}^{-1}$ depending on the volume dependence of the quantity $k_{11 j} t^{j}$ and find 4 different cases:

1. $k_{11 j} t^{j}=0$ or $k_{11 j} t^{j} \simeq \frac{\tau_{1}^{1 / 2+3 \alpha / 2}}{\mathcal{V}^{\alpha}}, \alpha \geq 1 \Longrightarrow K_{11}^{-1} \simeq \tau_{1}^{2}$,

2. $k_{11 j} t^{j}=\frac{\tau_{1}^{1 / 2+3 \alpha / 2}}{\mathcal{V}^{\alpha}}, 0<\alpha<1 \Longrightarrow K_{11}^{-1} \simeq \mathcal{V}^{\alpha} \tau_{1}^{2-3 \alpha / 2}, 0<\alpha<1$,

3. $k_{11 j} t^{j} \simeq \sqrt{\tau_{1}} \Longrightarrow K_{11}^{-1} \simeq \mathcal{V} \sqrt{\tau_{1}}$,

4. $k_{11 j} t^{j} \simeq \mathcal{V}^{\alpha} \tau_{1}^{1 / 2-3 \alpha / 2}, \alpha>0 \Longrightarrow K_{11}^{-1} \simeq \mathcal{V}^{\alpha} \tau_{1}^{2-3 \alpha / 2}, \alpha>1$.

One could wonder why we are setting the conditions of the Theorem on the elements of the inverse Kähler matrix and not on the intersection numbers or the form of the overall volume of the Calabi-Yau from which it would be easier to understand their topological meaning. The reason is that it is the inverse Kähler matrix which enters directly with the superpotential into the form of the scalar potential which is the one that determines the physics.

Moreover, the Theorem applies if the superpotential has the expression (2.11), but in this case we can still make linear field redefinitions that will not change $W$, corresponding to proper changes of basis, of the form

$$
\left\{\begin{array}{c}
\tau_{j} \longrightarrow \tau_{j}^{\prime}=\tau_{j}, \forall j=1, \ldots, N_{\text {small }}, \\
\tau_{j} \longrightarrow \tau_{j}^{\prime}=\tau_{j}+g_{1}\left(\tau_{i}\right), \forall j=N_{\text {small }}+1, \ldots, h_{1,1}(X),
\end{array}\right.
$$

where $g_{1}\left(\tau_{i}\right), i=1, \ldots, N_{\text {small }}$, is an homogeneous function of degree 1 . This means that the small 4-cycles will stay small and the large ones will just be perturbed by the small ones. We are therefore in the same situation and the physics should not change. We conclude that the inverse Kähler matrix should not change but both the intersection numbers and the form of the volume can indeed vary. In fact, for an arbitrary Calabi-Yau, the elements of the inverse Kähler matrix are given by:

$$
K_{i j}^{-1}=-\frac{4}{9} \mathcal{V} k_{i j k} t^{k}+4 \tau_{i} \tau_{j}
$$


and so we see that in order to keep the form of $K_{i j}^{-1}$ unaltered, the quantity $\left(k_{i j k} t^{k}\right)$ has not to vary, but the intersection numbers $k_{i j k}$ can indeed change. This is the main reason why we need to put our conditions on the $K_{i j}^{-1}$.

Let us illustrate this statement in the explicit example of the orientifold of the CalabiYau threefold $\mathbb{C} P_{[1,1,1,6,9]}^{4}$ whose volume in terms of 2 -cycle volumes is given by

$$
\mathcal{V}=6\left(t_{5}^{3}+t_{4}^{3}\right)
$$

The corresponding 4-cycle volumes look like

$$
\left\{\begin{array} { l } 
{ \tau _ { 4 } = \frac { \partial \mathcal { V } } { \partial t _ { 4 } } = 1 8 t _ { 4 } ^ { 2 } , } \\
{ \tau _ { 5 } = \frac { \partial \mathcal { V } } { \partial t _ { 5 } } = 1 8 t _ { 5 } ^ { 2 } , }
\end{array} \Longleftrightarrow \left\{\begin{array}{l}
t_{4}=-\frac{\sqrt{\tau_{4}}}{3 \sqrt{2}}, \\
t_{5}=+\frac{\sqrt{\tau_{5}}}{3 \sqrt{2}},
\end{array}\right.\right.
$$

and the volume in terms of the 4-cycles is

$$
\mathcal{V}=\frac{1}{9 \sqrt{2}}\left(\tau_{5}^{3 / 2}-\tau_{4}^{3 / 2}\right)
$$

Finally the superpotential reads

$$
W=W_{0}+A_{4} e^{-a_{4} T_{4}}+A_{5} e^{-a_{5} T_{5}} .
$$

It exists a well defined large volume limit when the 4-cycle $\tau_{4}$ is kept small and $\tau_{5}$ is sent to infinity. In this case the superpotential can be approximated as

$$
W \simeq W_{0}+A_{4} e^{-a_{4} T_{4}} .
$$

We can now perform the following field redefinition

$$
\left\{\begin{array}{c}
\tau_{4} \longrightarrow \tau_{4}^{\prime}=\tau_{4}, \\
\tau_{5} \longrightarrow \tau_{5}^{\prime}=\tau_{5}+\tau_{4},
\end{array}\right.
$$

which will not change the form of $W$ (A.25). However now the volume reads

$$
\mathcal{V}^{\prime}=\frac{1}{9 \sqrt{2}}\left(\left(\tau_{5}^{\prime}-\tau_{4}^{\prime}\right)^{3 / 2}-\tau_{4}^{\prime 3 / 2}\right) \simeq \frac{1}{9 \sqrt{2}}\left(\tau_{5}^{\prime 3 / 2}-\tau_{4}^{\prime} \sqrt{\tau_{5}^{\prime}}-\tau_{4}^{\prime 3 / 2}\right)
$$

which is clearly different from the initial form (A.23). This means that also the intersection numbers are different. However the elements of the inverse Kähler matrix do not change. In particular we are interested in $K_{44}^{-1} \simeq \mathcal{V} \sqrt{\tau_{4}}$ in this case as $\tau_{4}$ is the small cycle. Its form stays unchanged since $K_{44}^{\prime-1} \simeq \mathcal{V}^{\prime} \sqrt{\tau_{4}^{\prime}}$. From (A.20), this implies that

$$
\sqrt{\tau_{4}^{\prime}}=\left(k_{44 k}^{\prime} t^{\prime k}\right)=k_{444}^{\prime} t_{4}^{\prime}+k_{445}^{\prime} t_{5}^{\prime}
$$

and one would tend to say that $k_{445}^{\prime}$ has to be zero but we know from (A.27) that this is definitely not the case. This means that the field redefinition (A.26) will have the corresponding redefinition of the 2-cycle volumes which will produce $t_{4}^{\prime}$ and $t_{5}^{\prime}$ that are both large 2-cycles but such that the combination $\left(k_{444}^{\prime} t_{4}^{\prime}+k_{445}^{\prime} t_{5}^{\prime}\right)$ stays small. This is the 
reason why the form of the inverse Kähler matrix is left invariant while the intersection numbers do vary. This can be rephrased by saying that if $\tau_{j}$ is a small 4 -cycle, in general the corresponding $t_{j}$ has not to be a small 2-cycle and viceversa. This is clear without the need to perform any field redefinition in the case of the Calabi-Yau K3 fibration described by the degree 12 hypersurface in $\mathbb{C} P_{[1,1,2,2,6]}^{4}$ whose overall volume in terms of 2 -cycle volumes is

$$
\mathcal{V}=t_{1} t_{2}^{2}+\frac{2}{3} t_{2}^{3}
$$

giving relations between the 2- and 4-cycle volumes,

$$
\begin{aligned}
\tau_{1}=t_{2}^{2}, & \tau_{2}=2 t_{2}\left(t_{1}+t_{2}\right), \\
t_{2}=\sqrt{\tau_{1}}, & t_{1}=\frac{\tau_{2}-2 \tau_{1}}{2 \sqrt{\tau_{1}}},
\end{aligned}
$$

that allow us to write

$$
\mathcal{V}=\frac{1}{2} \sqrt{\tau_{1}}\left(\tau_{2}-\frac{2}{3} \tau_{1}\right)
$$

Looking at (A.31) we see that the large volume limit can be performed keeping $\tau_{1}$ small and taking $\tau_{2}$ large. Nonetheless, as it is clear from (A.30), $t_{1}$ is big whereas $t_{2}$ is small. Therefore it is impossible to impose that the quantity $k_{j j i} t^{i}$ does not introduce any volume dependence by requiring that some intersection numbers have to vanish.

Going back to the proof of the LARGE Volume Claim for $N_{\text {small }}=1$, let us assume that we are in case (3), so that (A.15) becomes

$$
V \simeq \frac{\sqrt{\tau_{1}}}{\mathcal{V}} A_{1}^{2} a_{1}^{2} e^{-2 a_{1} \tau_{1}}-\frac{W_{0}}{\mathcal{V}^{2}} A_{1} a_{1} \tau_{1} e^{-a_{1} \tau_{1}}+\frac{\hat{\xi}}{\mathcal{V}^{3}} W_{0}^{2},
$$

and when we take the decompactification limit given by

$$
\mathcal{V} \rightarrow \infty \text { with } e^{a_{1} \tau_{1}}=\frac{\mathcal{V}}{W_{0}}
$$

all the terms in (A.32) have the same volume dependence

$$
V \simeq \frac{W_{0}^{2}}{\mathcal{V}^{3}}\left[\left(A_{1} a_{1}-\sqrt{\tau_{1}}\right) A_{1} a_{1} \sqrt{\tau_{1}}+\hat{\xi}\right] .
$$

We can finally express the scaling behaviour of (A.34) as

$$
V \simeq \frac{W_{0}^{2}}{\mathcal{V}^{3}}\left(C_{1} \sqrt{\ln \mathcal{V}}-C_{2} \ln \mathcal{V}+\hat{\xi}\right)
$$

where $C_{1}$ and $C_{2}$ are positive constants of order 1 for natural values of the parameter $A_{1} \simeq 1$. We conclude that at large volume, the dominant term in (A.35) is the second one and the scalar potential approaches zero from below. It is now straightforward to argue that there must exist an exponentially large volume AdS minimum.

In fact, at smaller volumes the dominant term in the potential (A.35) is either the first or the third term, depending on the exact value of the constants. Both are positive as we have explained above. Thus at smaller volumes the potential is positive, and so since it 
must go to zero at infinity from below, there must exist a local AdS minimum along the direction in Kähler moduli space where the volume changes.

One could argue that if at smaller volumes the dominant term in (A.35) is the first one, then there is no need to require $h_{2,1}(X)>h_{1,1}(X)$. In reality this is wrong, because $\xi<0$ could still ruin the presence of the large volume minimum. In fact we can rewrite the full scalar potential (A.32) as

$$
V=\frac{\lambda}{\mathcal{V}} \sqrt{\tau_{1}} e^{-2 a_{1} \tau_{1}}-\frac{\mu}{\mathcal{V}^{2}} \tau_{1} e^{-a_{1} \tau_{1}}+\frac{\hat{\xi} W_{0}^{2}}{\mathcal{V}^{3}}
$$

where $\lambda, \mu$ and $\nu$ are positive constants depending on the exact details of this model. We can integrate out $\tau_{1}$, so ending up with just a potential for $\mathcal{V}$. Under the requirement $a_{1} \tau_{1} \gg 1, \partial V / \partial \tau_{1}=0$ gives

$$
e^{-a_{1} \tau_{1}}=\frac{\mu}{2} \frac{\sqrt{\tau_{1}}}{\lambda \mathcal{V}}
$$

which substituted back in (A.36) yields

$$
V=-\frac{1}{2} \frac{\mu^{2}}{\lambda} \frac{\tau_{1}^{3 / 2}}{\mathcal{V}^{3}}+\frac{\hat{\xi} W_{0}^{2}}{\mathcal{V}^{3}} \sim \frac{-(\ln \mathcal{V})^{3 / 2}+\hat{\xi} W_{0}^{2}}{\mathcal{V}^{3}}
$$

and is straightforward to see that we need $\hat{\xi}>0$ even though the dominant term at small volumes in (A.36) is the first one.

It remains to show that the scalar potential has also a minimum in the other direction of the moduli space. In order to do that, let us fix the Calabi-Yau volume and see what happens if we vary the small Kähler modulus along that surface. Then as one approaches the walls of the Kähler cone the positive first term in (A.32) dominates since it has the fewest powers of volume in the denominator and the exponential contributions of the modulus that is becoming small cannot be neglected. Thus at large overall volume, we expect the potential to grow in the positive direction towards the walls of the Kähler cone.

On the other hand, when the small Kähler modulus becomes bigger then the dominant term in (A.32) is the positive $V_{\left(\alpha^{\prime}\right)}$ due to the exponential suppressions in the other two terms. Given that the potential is negative along the special direction in the moduli space that we have identified and eventually raises to be positive or to vanish in the other direction, we are sure to have an AdS exponentially large volume minimum.

Since $V \sim \mathcal{O}\left(1 / \mathcal{V}^{3}\right)$ at the minimum, while $-3 e^{K}|W|^{2} \sim \mathcal{O}\left(1 / \mathcal{V}^{2}\right)$, it is clear that this minimum is non-supersymmetric. We can heuristically see why the minimum we are arguing for can be at exponentially large volume. The naive measure of its location is the value of the volume at which the negative term in (A.35) becomes dominant. As this occurs only when $(\ln \mathcal{V})$ is large, we expect to find the vacuum at large values of $(\ln \mathcal{V})$.

In reality the way in which we have taken the limit (A.33), tells us how the volume will scale, even though this can very well not be the correct location of the minimum

$$
\mathcal{V} \sim W_{0} e^{a_{1} \tau_{1}}
$$

Looking at (A.39) we realise that $W_{0}$ cannot be too small, otherwise we would get a small volume minimum merging with the KKLT one and our derivation would not make sense 
anymore. However $W_{0}$ is multiplying an exponential, which means that in order to destroy the large volume minimum $W_{0}$ has to be really small.

Furthermore, we stress that there is no need to require $h_{2,1}(X) \gg h_{1,1}(X)$ instead of just $h_{2,1}(X)>h_{1,1}(X)$. In fact, in this proof we have used $\hat{\xi}$ instead of $\xi$, so obscuring the presence of any factors of $g_{s}$ but, as it is written explicitly in (2.11), in Einstein frame $\hat{\xi}$ is equivalent to $\xi / g_{s}^{3 / 2}$. Therefore if we just have $h_{2,1}(X)>h_{1,1}(X)$ then we can still adjust $g_{s}$ to make sure that the AdS minimum is indeed at large volume.

We are now able to understand what happens if $K_{11}^{-1}$ is not in case (3). For example, when it is in case (4) then the first term in (A.15) beats all the other ones and along the direction (A.33) the scalar potential either presents a runaway or has no minimum at large volume depending on the exact value of $\alpha$.

Moreover if $K_{11}^{-1}$ is in case (1) or (2) then the first term in (A.15) is subleading with respect to the other two and at leading order in the volume, the scalar potential looks like

$$
V \simeq-\frac{W_{0}}{\mathcal{V}^{2}} A_{1} a_{1} \tau_{1} e^{-a_{1} \tau_{1}}+\frac{\hat{\xi}}{\mathcal{V}^{3}} W_{0}^{2}
$$

The minimisation equation for $\tau_{1}, \partial V / \partial \tau_{1}=0$, admits the only possible solution $a_{1} \tau_{1}=$ 1 , that has to be discarded since we need $a_{1} \tau_{1} \gg 1$ in order to avoid higher instanton corrections.

Finally, let us argue in favour of the last statement of Claim 1. At the end of all our derivation we realised that the small Kähler modulus $\tau_{1}$ plus a particular combination which is the overall volume are stabilised. Therefore we have in general $\left(N_{\text {small }}+1\right)$ fixed Kähler moduli and is straightforward to see that if we have just one big Kähler modulus then it will be fixed, whereas if we have more than one big Kähler moduli, only one of them will be fixed and the others will give rise to exactly $\left(h_{1,1}(X)-N_{\text {small }}-1\right)$ flat directions. This is because they do not appear in the non-perturbative corrections to the superpotential due to the limit 2.10). This terminates our proof of the LARGE Volume Claim for $N_{\text {small }}=1$.

\section{A.2 Proof for $N_{\text {small }}>1$}

Proof. (LARGE Volume Claim for $N_{\text {small }}>1$ ) When $N_{\text {small }}>1$ the situation is more involved due to the presence of cross terms. However $V_{\left(\alpha^{\prime}\right)}$ has still the form (A.2). Without loss of generality, we shall focus on the case with $N_{\text {small }}=2$ Kähler moduli, which we will call $\tau_{1}$ and $\tau_{2}$. $V_{n p 1}$ generalises to

$$
\begin{aligned}
& V_{n p 1} \underset{\mathcal{V} \rightarrow \infty}{\longrightarrow} e^{K} \sum_{j, k=1}^{2} K_{j k}^{-1} a_{j} A_{j} a_{k} \bar{A}_{k} e^{-\left(a_{j} T_{j}+a_{k} \bar{T}_{k}\right)} \\
= & e^{K}\left\{\sum_{j=1}^{2} K_{j j}^{-1} a_{j}^{2}\left|A_{j}\right|^{2} e^{-2 a_{j} \tau_{j}}+K_{12}^{-1} a_{1} A_{1} a_{2} \bar{A}_{2} e^{-\left(a_{1} \tau_{1}+a_{2} \tau_{2}\right)} e^{i\left(a_{2} b_{2}-a_{1} b_{1}\right)}\right\} .
\end{aligned}
$$

In order to consider separately the axion-dependent part of $V_{n p 1}$, we write

$$
V_{n p 1}=V_{n p 1}^{r e a l}+V_{n p 1}^{A X}
$$


Switching to the study of $V_{n p 2}$, we find that

$$
V_{n p 2} \underset{\mathcal{V} \rightarrow \infty}{\longrightarrow}-e^{K} \sum_{k=1}^{h_{1,1}} \sum_{j=1}^{2} K_{j k}^{-1}\left[\left(a_{j} A_{j} e^{-a_{j} \tau_{j}} e^{-i a_{j} b_{j}} \bar{W} \partial_{\bar{T}_{k}} K\right)+\left(a_{j} \bar{A}_{j} e^{-a_{j} \tau_{j}} e^{+i a_{j} b_{j}} W \partial_{T_{k}} K\right)\right]
$$

where we have used the fact that $K_{j k}^{-1}=K_{k j}^{-1}$. Equation (A.43) can be rewritten as

$$
\begin{aligned}
& V_{n p 2} \underset{\mathcal{V} \rightarrow \infty}{\longrightarrow}-e^{K} \sum_{k=1}^{h_{1,1}} \sum_{j=1}^{2} K_{j k}^{-1}\left(\partial_{T_{k}} K\right) a_{j} e^{-a_{j} \tau_{j}}\left[\left(A_{j} \bar{W} e^{-i a_{j} b_{j}}\right)+\left(\bar{A}_{j} W e^{+i a_{j} b_{j}}\right)\right] \\
= & \sum_{j=1}^{2}\left(X_{j} e^{+i a_{j} b_{j}}+\bar{X}_{j} e^{-i a_{j} b_{j}}\right),
\end{aligned}
$$

where

$$
X_{j} \equiv-e^{K} K_{j k}^{-1}\left(\partial_{T_{k}} K\right) a_{j} \bar{A}_{j} W e^{-a_{j} \tau_{j}}
$$

We note that for a general Calabi-Yau, the following relation holds:

$$
K_{j k}^{-1}\left(\partial_{T_{k}} K\right)=-2 \tau_{j}
$$

and thus the definition $(\mathrm{A} .45)$ can be simplified to

$$
X_{j} \equiv 2 e^{K} a_{j} \tau_{j}\left|A_{j}\right| e^{-i \vartheta_{j}}|W| e^{i \vartheta_{W}} e^{-a_{j} \tau_{j}}=\left|X_{j}\right| e^{i\left(\vartheta_{W}-\vartheta_{j}\right)} .
$$

Therefore

$$
V_{n p 2} \underset{\mathcal{V} \rightarrow \infty}{\longrightarrow} \sum_{j=1}^{2}\left|X_{j}\right|\left(e^{+i\left(\vartheta_{W}-\vartheta_{j}+a_{j} b_{j}\right)}+e^{-i\left(\vartheta_{W}-\vartheta_{j}+a_{j} b_{j}\right)}\right)
$$

Let us now reconsider $V_{n p 1}^{A X}$, which we had set aside for a moment. It can be rewritten as

$$
V_{n p 1}^{A X}=e^{K} K_{12}^{-1} a_{1} a_{2} e^{-\left(a_{1} \tau_{1}+a_{2} \tau_{2}\right)}\left(A_{1} \bar{A}_{2} e^{i\left(a_{2} b_{2}-a_{1} b_{1}\right)}+A_{2} \bar{A}_{1} e^{-i\left(a_{2} b_{2}-a_{1} b_{1}\right)}\right),
$$

and finally as

$$
V_{n p 1}^{A X}=Y_{12} e^{i\left(a_{2} b_{2}-a_{1} b_{1}\right)}+\bar{Y}_{12} e^{-i\left(a_{2} b_{2}-a_{1} b_{1}\right)}
$$

where

$$
Y_{12} \equiv e^{K} K_{12}^{-1} a_{1} a_{2} A_{1} \bar{A}_{2} e^{-\left(a_{1} \tau_{1}+a_{2} \tau_{2}\right)}=\left|Y_{12}\right| e^{i\left(\vartheta_{1}-\vartheta_{2}\right)}
$$

Therefore

$$
V_{n p 1}^{A X}=\left|Y_{12}\right|\left(e^{i\left(\vartheta_{1}-\vartheta_{2}+a_{2} b_{2}-a_{1} b_{1}\right)}+e^{-i\left(\vartheta_{1}-\vartheta_{2}+a_{2} b_{2}-a_{1} b_{1}\right)}\right) .
$$

Thus, the full axion-dependent part of the scalar potential $V_{A X}$ looks like

$$
V_{A X}=V_{n p 2}+V_{n p 1}^{A X}=2 \sum_{j=1}^{2}\left|X_{j}\right| \cos \left(\vartheta_{W}-\vartheta_{j}+a_{j} b_{j}\right)+2\left|Y_{12}\right| \cos \left(\vartheta_{1}-\vartheta_{2}+a_{2} b_{2}-a_{1} b_{1}\right) .
$$




\section{A.2.1 Axion stabilisation}

$V_{A X}$ is a scalar function of the axions $b_{1}$ and $b_{2}$ whereas $\vartheta_{1}, \vartheta_{2}$ and $\vartheta_{W}$ are to be considered just as parameters. In order to find a minimum for $V_{A X}$ let us set its gradient to zero

$$
\left\{\begin{array}{cc}
\partial V_{A X} / \partial b_{1}=0 & \Longleftrightarrow\left|X_{1}\right| \sin \left(\vartheta_{W}-\vartheta_{1}+a_{1} b_{1}\right)=+\left|Y_{12}\right| \sin \left(\vartheta_{1}-\vartheta_{2}+a_{2} b_{2}-a_{1} b_{1}\right), \\
\partial V_{A X} / \partial b_{2}=0 & \Longleftrightarrow\left|X_{2}\right| \sin \left(\vartheta_{W}-\vartheta_{2}+a_{2} b_{2}\right)=-\left|Y_{12}\right| \sin \left(\vartheta_{1}-\vartheta_{2}+a_{2} b_{2}-a_{1} b_{1}\right),
\end{array}\right.
$$

The solution of (A.54) is given by

$$
\begin{cases}\psi_{1} \equiv\left(\vartheta_{W}-\vartheta_{1}+a_{1} b_{1}\right)=p_{1} \pi, & p_{1} \in \mathbb{Z} \\ \psi_{2} \equiv\left(\vartheta_{W}-\vartheta_{2}+a_{2} b_{2}\right)=p_{2} \pi, & p_{2} \in \mathbb{Z}\end{cases}
$$

and

$$
\psi_{12} \equiv\left(\vartheta_{1}-\vartheta_{2}+a_{2} b_{2}-a_{1} b_{1}\right)=p_{12} \pi, \quad p_{12} \in \mathbb{Z} .
$$

From (A.55) equation A.56) requires $p_{12}=p_{2}-p_{1}$. Let us summarise the points where the gradient of the axion potential is zero in the following table

\begin{tabular}{|c|c|c|c|c|}
\hline & $(\mathrm{a})$ & $(\mathrm{b})$ & $(\mathrm{c})$ & $(\mathrm{d})$ \\
\hline $\cos \psi_{1}$ & +1 & -1 & +1 & -1 \\
\hline $\cos \psi_{2}$ & +1 & -1 & -1 & +1 \\
\hline $\cos \psi_{12}$ & +1 & +1 & -1 & -1 \\
\hline
\end{tabular}

We notice that the phases of $W, A_{1}$ and $A_{2}$ will not enter into $V_{n p 2}$ once the axions have been properly minimised and so, without loss of generality, we can consider $W, A_{1}$ and $A_{2} \in \mathbb{R}^{+}$from now on.

We have still to check the Hessian matrix evaluated at $b_{1}$ and $b_{2}$ as given in (A.55) and require it to be positive definite. Its diagonal elements are given by

$$
\left\{\begin{array}{l}
\partial^{2} V_{A X} / \partial b_{1}^{2}=-2 a_{1}^{2}\left(\left|X_{1}\right| \cos \psi_{1}+\left|Y_{12}\right| \cos \psi_{12}\right) \\
\partial^{2} V_{A X} / \partial b_{2}^{2}=-2 a_{2}^{2}\left(\left|X_{2}\right| \cos \psi_{2}+\left|Y_{12}\right| \cos \psi_{12}\right)
\end{array}\right.
$$

whereas the non-diagonal ones read

$$
\frac{\partial^{2} V_{A X}}{\partial b_{2} \partial b_{1}}=\frac{\partial^{2} V_{A X}}{\partial b_{1} \partial b_{2}}=2 a_{1} a_{2}\left|Y_{12}\right| \cos \psi_{12}
$$

We can diagonalise the Hessian $\mathcal{H}$ to the identity by decomposing it a la Choleski into $\mathcal{H}=\mathcal{U}^{T} \mathbb{U}$, where the elements of the upper triangular matrix $\mathcal{U}$ are given by the following recursive relations:

$$
\begin{gathered}
\mathcal{U}_{11}^{2}=-2 a_{1}^{2}\left(\left|X_{1}\right| \cos \psi_{1}+\left|Y_{12}\right| \cos \psi_{12}\right), \\
\mathcal{U}_{12}=\frac{2 a_{1} a_{2}\left|Y_{12}\right| \cos \psi_{12}}{\mathcal{U}_{11}}, \\
\mathcal{U}_{22}^{2}=-\mathcal{U}_{12}^{2}-2 a_{2}^{2}\left(\left|X_{2}\right| \cos \psi_{2}+\left|Y_{12}\right| \cos \psi_{12}\right),
\end{gathered}
$$

with $\mathcal{U}_{21}=0$. Determining if the Hessian is positive definite is equal to checking that $\mathcal{U}$ is a real matrix. Looking at A.61) we realise that $\mathcal{U}_{12}$ is automatically real if $\mathcal{U}_{11}$ is real. Hence we have to make sure just that both $\mathcal{U}_{11}^{2}>0$ and $\mathcal{U}_{22}^{2}>0$. When we analyse the cases listed in table (A.57) we realise that: 
(a) can never be a minimum since $\mathcal{U}_{11}^{2}<0$; in reality it turns out to be always a maximum,

(b) is a minimum only if $\left|X_{1}\right|>\left|Y_{12}\right|$ and $\left|X_{1}\right|\left|X_{2}\right|>\left|Y_{12}\right|\left(\left|X_{1}\right|+\left|X_{2}\right|\right)$,

(c) is a minimum only if $\left|Y_{12}\right|>\left|X_{1}\right|$ and $\left|X_{2}\right|\left|Y_{12}\right|>\left|X_{1}\right|\left(\left|X_{2}\right|+\left|Y_{12}\right|\right)$,

(d) is a minimum only if $\left|Y_{12}\right|\left|X_{1}\right|>\left|X_{2}\right|\left(\left|X_{1}\right|+\left|Y_{12}\right|\right)$,

where, according to the definitions (A.47) and (A.51), we have

$$
\left\{\begin{array}{c}
\left|X_{1}\right|=2\left|A_{1}\right| a_{1} \tau_{1}|W| \frac{e^{-a_{1} \tau_{1}}}{\mathcal{V}^{2}}, \\
\left|X_{2}\right|=2\left|A_{2}\right| a_{2} \tau_{2}|W| \frac{e^{-a_{2} \tau_{2}}}{\mathcal{V}^{2}}, \\
\left|Y_{12}\right|=K_{12}^{-1}\left|A_{1}\right| a_{1}\left|A_{2}\right| a_{2} \frac{e^{-a_{1} \tau_{1}} e^{-a_{2} \tau_{2}}}{\mathcal{V}^{2}} .
\end{array}\right.
$$

In order to study the cases (b), (c) and (d), it is therefore crucial to know the order of magnitude of the two exponentials $e^{-a_{1} \tau_{1}}$ and $e^{-a_{2} \tau_{2}}$ given by their scaling behaviour in the volume. This depends on the direction we are looking at to find the minimum in the large volume limit which can be performed in three different ways:

$$
\begin{aligned}
I) \mathcal{V} & \sim e^{\gamma a_{1} \tau_{1}} \sim e^{\gamma a_{2} \tau_{2}}, \quad \gamma \in \mathbb{R}^{+} \\
I I) \mathcal{V} \sim e^{\beta a_{1} \tau_{1}} & \sim e^{\gamma a_{2} \tau_{2}}, \quad \beta<\gamma, \quad \gamma, \beta \in \mathbb{R}^{+}, \\
I I I) \mathcal{V} \sim e^{\beta a_{1} \tau_{1}} & \sim e^{\gamma a_{2} \tau_{2}}, \quad \beta>\gamma \quad \gamma, \beta \in \mathbb{R}^{+}
\end{aligned}
$$

Finally we need also to know the form of $K_{12}^{-1}$. We can classify its behaviour according to the volume dependence of the quantity $k_{12 j} t^{j}$ and find 4 different cases:

1. $k_{12 j} t^{j}=0$ or $k_{12 j} t^{j}=\frac{f\left(\tau_{1}, \tau_{2}\right)}{\mathcal{V}^{\alpha}}, \alpha \geq 1 \Longrightarrow K_{12}^{-1}=\tau_{1} \tau_{2}$;

2. $k_{12 j} t^{j}=\frac{g_{\gamma}\left(\tau_{1}, \tau_{2}\right)}{\mathcal{V}^{\alpha}}, 0<\alpha<1, g$ homogeneous function of degree $\gamma=\frac{1+3 \alpha}{2} \Longrightarrow K_{12}^{-1}=$ $\mathcal{V}^{\alpha} g_{2-3 \alpha / 2}\left(\tau_{1}, \tau_{2}\right), \quad 0<\alpha<1$;

3. $k_{12 j} t^{j}=f_{1 / 2}\left(\tau_{1}, \tau_{2}\right), f$ homogeneous function of degree $1 / 2 \Longrightarrow K_{12}^{-1}=\mathcal{V} f_{1 / 2}\left(\tau_{1}, \tau_{2}\right)$;

4. $k_{12 j} t^{j}=\mathcal{V}^{\alpha} h_{\beta}\left(\tau_{1}, \tau_{2}\right), \alpha>0, h$ homogeneous function of degree $\beta=\frac{1-3 \alpha}{2} \Longrightarrow K_{12}^{-1}=$ $\mathcal{V}^{\alpha} h_{2-3 \alpha / 2}\left(\tau_{1}, \tau_{2}\right), \quad \alpha>1$.

Let us now focus on the axion minimisation by analysing each of these 4 cases in full detail. For each case we will have to study if the inequalities (b), (c) and (d) admit a solution for any of the three possible ways to take the large volume limit as expressed in (A.64). We will always consider natural values of the parameters $\left|A_{1}\right| \simeq\left|A_{2}\right| \simeq|W| \simeq 1$.

From (I) of (A.64), we can immediately realise that, regardless of the form of $K_{12}^{-1}$, at large volume $\left|X_{1}\right|$ and $\left|X_{2}\right|$ have the same scaling with the volume and so we can denote both of them as $|X|$. It is then straightforward to see that both the second (c)-condition and the $(d)$-condition can never be satisfied. In fact they take the form

$$
|X|\left|Y_{12}\right|>|X|\left|Y_{12}\right|+|X|^{2},
$$


which is manifestly an absurd. This implies that neither (c) nor (d) can be a minimum along the direction (I) for any value of $K_{12}^{-1}$. A further analysis reveals that the points (c) and (d) can never be maxima so since we proved that they cannot be minima, they are forced to be saddle points. We do not present the details of this analysis here since it is not important for our reasoning. On the other hand, the first (b)-condition is automatically satisfied if the second one is true since it reduces to

$$
|X|^{2}>2|X|\left|Y_{12}\right| \Longleftrightarrow|X|>2\left|Y_{12}\right|
$$

From (II) of (A.64), we also notice that, regardless of the form of $K_{12}^{-1}$, at large volume $\left|X_{1}\right|<\left|X_{2}\right|$ since $\beta<\gamma$. It is then straightforward to see that in this situation the (d)condition can never be satisfied. This implies that (d) is always a saddle point along the direction (II) for any value of $K_{12}^{-1}$.

Furthermore (III) of (A.64) implies that, regardless of the form of $K_{12}^{-1}$, at large volume $\left|X_{1}\right|>\left|X_{2}\right|$ as $\beta>\gamma$. Then we immediately see that the second (c)-condition can never be satisfied. Therefore (c) is always a saddle point along the direction (III) for any value of $K_{12}^{-1}$.

Case (1): $K_{12}^{-1} \simeq \tau_{1} \tau_{2}$

- $\operatorname{direction}(\mathrm{I})$

The volume dependence of the parameters $(\mathrm{A.63})$ is

$$
\left\{\begin{array}{l}
\left|X_{1}\right| \simeq\left|X_{2}\right| \simeq \mathcal{V}^{-(2+1 / \gamma)} \ln \mathcal{V} \\
\left|Y_{12}\right| \simeq \mathcal{V}^{-(2+2 / \gamma)}(\ln \mathcal{V})^{2}
\end{array}\right.
$$

Looking at (A.67), we realise that at large volume $\left|X_{1}\right|>2\left|Y_{12}\right|$. Therefore the second (b)-condition (A.66) is satisfied and (b) is a minimum of the axion potential.

- direction (II)

The parameters (A.63) now read

$$
\left\{\begin{array}{l}
\left|X_{1}\right| \simeq \mathcal{V}^{-(2+1 / \beta)} \ln \mathcal{V} \\
\left|X_{2}\right| \simeq \mathcal{V}^{-(2+1 / \gamma)} \ln \mathcal{V} \\
\left|Y_{12}\right| \simeq \mathcal{V}^{-(2+1 / \beta+1 / \gamma)}(\ln \mathcal{V})^{2}
\end{array}\right.
$$

Looking at (A.68), we realise that at large volume $\left|X_{1}\right|>\left|Y_{12}\right|$, which implies that (c) is a saddle point. Thus the first (b)-condition is satisfied and the second becomes

$$
\frac{(\ln \mathcal{V})^{2}}{\mathcal{V}^{4+1 / \beta+1 / \gamma}}>\frac{(\ln \mathcal{V})^{2}}{\mathcal{V}^{2+1 / \beta+1 / \gamma}}\left(\frac{\ln \mathcal{V}}{\mathcal{V}^{2+1 / \beta}}+\frac{\ln \mathcal{V}}{\mathcal{V}^{2+1 / \gamma}}\right) \underset{1 / \beta>1 / \gamma}{\simeq} \frac{(\ln \mathcal{V})^{3}}{\mathcal{V}^{4+1 / \beta+2 / \gamma}}
$$

which is true at large volume for values of $\gamma>\beta>0$ not extremely big. Thus (b) is a minimum of the axion potential. 
- direction (III)

The parameters (A.63) take the same form as (A.68) but now with $\beta>\gamma$. We have still $\left|X_{1}\right|>\left|Y_{12}\right|$, which implies that the first (b)-condition is satisfied. The second looks like

$$
\frac{(\ln \mathcal{V})^{2}}{\mathcal{V}^{4+1 / \beta+1 / \gamma}}>\frac{(\ln \mathcal{V})^{2}}{\mathcal{V}^{2+1 / \beta+1 / \gamma}}\left(\frac{\ln \mathcal{V}}{\mathcal{V}^{2+1 / \beta}}+\frac{\ln \mathcal{V}}{\mathcal{V}^{2+1 / \gamma}}\right) \underset{1 / \beta<1 / \gamma}{\simeq} \frac{(\ln \mathcal{V})^{3}}{\mathcal{V}^{4+2 / \beta+1 / \gamma}}
$$

which is true at large volume. Thus (b) is a minimum of the axion potential. On the contrary the simplified $(\mathrm{d})$-condition reads

$$
\frac{\ln \mathcal{V}}{\mathcal{V}^{1 / \beta}}>1+\frac{\ln \mathcal{V}}{\mathcal{V}^{1 / \gamma}}
$$

which at large volume is clearly false for values of $\beta>\gamma>0$ not extremely big. It follows that in this case (d) is a saddle point.

Let us summarise the results found in case (1) in the following table

\begin{tabular}{|c|c|c|c|}
\hline & (I) & (II) & (III) \\
\hline (a) & max & $\max$ & $\max$ \\
\hline (b) & min & min & min \\
\hline (c) & saddle & saddle & saddle \\
\hline (d) & saddle & saddle & saddle \\
\hline
\end{tabular}

Case (2): $K_{12}^{-1}=\mathcal{V}^{\alpha} g_{2-3 \alpha / 2}\left(\tau_{1}, \tau_{2}\right), \quad 0<\alpha<1$

- direction (I)

The volume dependence of the parameters (A.63) now reads

$$
\left\{\begin{array}{l}
\left|X_{1}\right| \simeq\left|X_{2}\right| \simeq \mathcal{V}^{-(2+1 / \gamma)} \ln \mathcal{V} \\
\left|Y_{12}\right| \simeq \mathcal{V}^{-(2+2 / \gamma-\alpha)}(\ln \mathcal{V})^{2-3 \alpha / 2}
\end{array}\right.
$$

Substituting the expressions $($ A.72 $)$ in $($ A.66 $)$ we find

$$
1>2 \frac{(\ln \mathcal{V})^{1-3 \alpha / 2}}{\mathcal{V}^{1 / \gamma-\alpha}}
$$

which at large volume is true if $\alpha<(1 / \gamma)$, false if $\alpha<(1 / \gamma)$ or $\alpha=(1 / \gamma) \leq 2 / 3$. On the contrary, for $2 / 3<\alpha=(1 / \gamma)<1$ the minimum is present. Thus (b) can be a minimum of the axion potential.

- $\operatorname{direction}(\mathrm{II})$

The parameters $(\mathrm{A.63})$ now read

$$
\left\{\begin{array}{l}
\left|X_{1}\right| \simeq \mathcal{V}^{-(2+1 / \beta)} \ln \mathcal{V} \\
\left|X_{2}\right| \simeq \mathcal{V}^{-(2+1 / \gamma)} \ln \mathcal{V} \\
\left|Y_{12}\right| \simeq \mathcal{V}^{-(2+1 / \beta+1 / \gamma-\alpha)}(\ln \mathcal{V})^{2-3 \alpha / 2}
\end{array}\right.
$$


The first (b)-condition becomes

$$
1>\frac{(\ln \mathcal{V})^{1-3 \alpha / 2}}{\mathcal{V}^{1 / \gamma-\alpha}}
$$

that is satisfied if either $\alpha<(1 / \gamma)$ or $2 / 3<\alpha=(1 / \gamma)<1$. Otherwise (A.75) is false unless $\alpha=(1 / \gamma)=2 / 3$ in which case we cannot conclude anything just looking at the volume dependence. However the second (b)-condition reads

$$
1>(\ln \mathcal{V})^{1-3 \alpha / 2}\left(\frac{1}{\mathcal{V}^{1 / \beta-\alpha}}+\frac{1}{\mathcal{V}^{1 / \gamma-\alpha}}\right)
$$

which is definitely true at large volume if $\alpha<(1 / \gamma)$ or $2 / 3<\alpha=(1 / \gamma)<1$. On the contrary, in the case $\alpha=(1 / \gamma)=2 / 3 \Leftrightarrow(1 / \beta-2 / 3)>0$, (A.76) becomes

$$
1>1+\frac{1}{\mathcal{V}^{1 / \beta-2 / 3}}
$$

which is clearly impossible. Thus (b) can be a minimum of the axion potential. We need now just to study the case (c) for which the first inequality is

$$
1<\frac{(\ln \mathcal{V})^{1-3 \alpha / 2}}{\mathcal{V}^{1 / \gamma-\alpha}}
$$

that is satisfied if either $(1 / \gamma)<\alpha<1$ or $\alpha=(1 / \gamma)<2 / 3$. Otherwise (A.78) is false unless $\alpha=(1 / \gamma)=2 / 3$ in which case we cannot conclude anything just looking at the volume dependence. However the second (c)-condition can be simplified to give

$$
\frac{(\ln \mathcal{V})^{1-3 \alpha / 2}}{\mathcal{V}^{1 / \gamma-\alpha}}>1+\frac{(\ln \mathcal{V})^{1-3 \alpha / 2}}{\mathcal{V}^{1 / \beta-\alpha}}
$$

which is clearly satisfied at large volume if either $(1 / \gamma)<\alpha<1$ or $\alpha=(1 / \gamma)<2 / 3$. On the contrary when $\alpha=(1 / \gamma)=2 / 3 \Leftrightarrow(1 / \beta-2 / 3)>0$, (A.79) becomes

$$
1>1+\frac{1}{\mathcal{V}^{1 / \beta-2 / 3}}
$$

which is clearly false.

- direction (III)

The parameters (A.63) assume the same form as (A.74) but now with $\beta>\gamma$. Following lines of reasoning similar to those used for direction (II), we get the results summarised in the following table along with all the others for case (2). 


\begin{tabular}{|c|c|c|c|}
\hline & (I) & (II) & (III) \\
\hline (a) & $\max$ & $\max$ & $\max$ \\
\hline (b) & $\begin{array}{c}\alpha<1 / \gamma \min \\
2 / 3<\alpha=1 / \gamma<1 \text { min } \\
\alpha=1 / \gamma \leq 2 / 3 \text { saddle } \\
\alpha>1 / \gamma \text { saddle. }\end{array}$ & $\left\{\begin{array}{c}\alpha<1 / \gamma \min \\
2 / 3<\alpha=1 / \gamma<1 \mathrm{~min} \\
\alpha=1 / \gamma \leq 2 / 3 \text { saddle } \\
\alpha>1 / \gamma \text { saddle }\end{array}\right.$ & $\begin{array}{c}\alpha<1 / \beta \min \\
2 / 3<\alpha=1 / \beta<1 \text { min } \\
\alpha=1 / \beta \leq 2 / 3, \text { saddle } \\
\alpha>1 / \beta \text { saddle }\end{array}$ \\
\hline (c) & saddle & $\begin{array}{c}\alpha<1 / \gamma \text { saddle } \\
2 / 3 \leq \alpha=1 / \gamma<1 \text { saddle } \\
\alpha=1 / \gamma<2 / 3 \text { min } \\
1 / \gamma<\alpha<1 \mathrm{~min}\end{array}$ & saddle \\
\hline (d) & saddle & saddle & $\begin{array}{c}\alpha<1 / \beta \text { saddle } \\
2 / 3 \leq \alpha=1 / \beta<1 \text { saddle } \\
\alpha=1 / \beta<2 / 3, \text { min } \\
\alpha>1 / \beta \text { min }\end{array}$ \\
\hline
\end{tabular}

Case (3): $K_{12}^{-1}=\mathcal{V} f_{1 / 2}\left(\tau_{1}, \tau_{2}\right)$

- direction (I)

The volume dependence of the parameters (A.63) now looks like

$$
\left\{\begin{array}{l}
\left|X_{1}\right| \simeq\left|X_{2}\right| \simeq \mathcal{V}^{-(2+1 / \gamma)} \ln \mathcal{V} \\
\left|Y_{12}\right| \simeq \mathcal{V}^{-(1+2 / \gamma)} \sqrt{\ln \mathcal{V}}
\end{array}\right.
$$

Substituting the expressions (A.81) in (A.66) we find

$$
\frac{\ln \mathcal{V}}{\mathcal{V}^{2+1 / \gamma}}>2 \frac{\sqrt{\ln \mathcal{V}}}{\mathcal{V}^{1+2 / \gamma}}
$$

which at large volume is true if $\gamma \leq 1$, false if $\gamma>1$. Thus (b) can be a minimum of the axion potential.

- $\operatorname{direction}(\mathrm{II})$

The parameters $(\mathrm{A} .63)$ now read

$$
\left\{\begin{array}{l}
\left|X_{1}\right| \simeq \mathcal{V}^{-(2+1 / \beta)} \ln \mathcal{V}, \\
\left|X_{2}\right| \simeq \mathcal{V}^{-(2+1 / \gamma)} \ln \mathcal{V}, \\
\left|Y_{12}\right| \simeq \mathcal{V}^{-(1+1 / \beta+1 / \gamma)} \sqrt{\ln \mathcal{V}}
\end{array}\right.
$$

Looking at (A.83), we realise that the first (b)-condition becomes

$$
\frac{\sqrt{\ln \mathcal{V}}}{\mathcal{V}}>\frac{1}{\mathcal{V}^{1 / \gamma}}
$$

which is satisfied only if $\gamma \leq 1$. Viceversa the first (c)-condition is satisfied only for $\gamma>1$. Let us check now the validity of the second (b)-condition which reads

$$
\frac{\sqrt{\ln \mathcal{V}}}{\mathcal{V}}>\frac{1}{\mathcal{V}^{1 / \beta}}+\frac{1}{\mathcal{V}^{1 / \gamma}}
$$


which, at large volume, is automatically true if $\gamma \leq 1$. The second (c)-condition is also correctly satisfied for $\gamma>1$ since it reads

$$
\frac{1}{\mathcal{V}^{1 / \gamma}}>\frac{\sqrt{\ln \mathcal{V}}}{\mathcal{V}}+\frac{1}{\mathcal{V}^{1 / \beta}}
$$

Thus both (b) and (c) can be a minimum of the axion potential.

- direction (III)

The parameters (A.63) assume the same form as (A.83) but now with $\beta>\gamma$. The inequality corresponding to the $(\mathrm{d})$-condition reads

$$
\frac{1}{\mathcal{V}^{1 / \beta}}>\frac{1}{\mathcal{V}^{1 / \gamma}}+\frac{\sqrt{\ln \mathcal{V}}}{\mathcal{V}}
$$

and becomes true if $\beta>1$. Moreover, the first (b)-condition (A.84) is again satisfied for $\gamma \leq 1$. On the other hand, the second looks like (A.85) and now, at large volume, is true only if $\beta \leq 1$, which implies correctly $\gamma \leq 1$ since in this case $\gamma<\beta$. It follows that both (b) and (d) can be a minimum.

Let us summarise the results found in case (3) in the following table

\begin{tabular}{|c|c|c|c|}
\hline & (I) & (II) & (III) \\
\hline (a) & max & $\max$ & $\max$ \\
\hline (b) & $\left\{\begin{array}{c}0<\gamma \leq 1 \mathrm{~min}, \\
\gamma>1 \text { saddle. }\end{array}\right.$ & $\left\{\begin{array}{c}0<\gamma \leq 1 \mathrm{~min}, \\
\gamma>1 \text { saddle. }\end{array}\right.$ & $\left\{\begin{array}{c}0<\gamma<\beta \leq 1 \mathrm{~min}, \\
\beta>1 \text { saddle. }\end{array}\right.$ \\
\hline (c) & saddle & $\left\{\begin{array}{c}0<\gamma \leq 1 \text { saddle, } \\
\gamma>1 \text { min. }\end{array}\right.$ & saddle \\
\hline (d) & saddle & saddle & $\left\{\begin{array}{c}0<\gamma<\beta \leq 1 \text { saddle, } \\
\beta>1 \text { min. }\end{array}\right.$ \\
\hline
\end{tabular}

Case (4): $K_{12}^{-1}=\mathcal{V}^{\alpha} h_{2-3 \alpha / 2}\left(\tau_{1}, \tau_{2}\right), \quad \alpha>1$

- $\operatorname{direction}(\mathrm{I})$

The volume dependence of the parameters (A.63) is given again by (A.72) and (A.66) takes the same form as the inequality (A.73) which at large volume is true if $\alpha<1 / \gamma$, false if $\alpha>1 / \gamma$. The situation $\alpha=1 / \gamma$ is more involved and A.73) simplifies to

$$
1>2(\ln \mathcal{V})^{1-3 \alpha / 2}
$$

which gives a positive result if $\alpha>2 / 3$. This is definitely true in our case where $\alpha>1$. Thus (b) can be a minimum of the axion potential.

- $\operatorname{direction}(\mathrm{II})$

The parameters (A.63) now take the same form given in (A.74). It follows then that the first (b)-condition $\left|X_{1}\right|>\left|Y_{12}\right|$ looks like (A.75) and is verified for $1 / \gamma \geq \alpha$. 
The second (b)-condition looks like (A.76) which at large volume is correctly true for $1 / \gamma \geq \alpha$. Thus (b) is a minimum of the axion potential. On the contrary the first (c)-condition implies $1 / \gamma<\alpha$, whereas the second is similar to the inequality (A.79) which is again clearly true for $1 / \gamma<\alpha$. It follows that in this case (c) can also be a minimum.

- direction (III)

The parameters (A.63) assume the same form as (A.74) but now with $\beta>\gamma$. The (d)-condition looks like

$$
\frac{(\ln \mathcal{V})^{1-3 \alpha / 2}}{\mathcal{V}^{1 / \beta-\alpha}}>1+\frac{(\ln \mathcal{V})^{1-3 \alpha / 2}}{\mathcal{V}^{1 / \gamma-\alpha}}
$$

and is verified only if $1 / \beta<\alpha$. On the other hand, the first (b)-condition is again given by (A.75) and so is still solved for $1 / \gamma \geq \alpha$. The second (b)-condition looks like (A.76) but now at large volume it is satisfied for $1 / \beta \geq \alpha$. It follows that in this case both (b) and (d) can be a minimum of the axion potential.

Let us summarise the results found in case (4) in the following table

\begin{tabular}{|c|c|c|c|}
\hline & (I) & (II) & (III) \\
\hline (a) & $\max$ & $\max$ & $\max$ \\
\hline (b) & $\begin{array}{c}1<\alpha \leq 1 / \gamma \min \\
\alpha>1 / \gamma \text { saddle. }\end{array}$ & $\begin{array}{c}1<\alpha \leq 1 / \gamma \min \\
\alpha>1 / \gamma \text { saddle }\end{array}$ & $\begin{array}{c}1<\alpha \leq 1 / \beta<1 / \gamma \min \\
1 / \beta<\alpha \text { saddle. }\end{array}$ \\
\hline (c) & saddle & $\begin{array}{c}1<\alpha \leq 1 / \gamma \text { saddle } \\
\alpha>1 / \gamma \min .\end{array}$ & saddle \\
\hline (d) & saddle & saddle & $\begin{array}{c}\alpha \leq 1 / \beta \text { saddle } \\
\alpha>1 / \beta \min .\end{array}$ \\
\hline
\end{tabular}

\section{A.2.2 Kähler moduli stabilisation}

After this long analysis of the axion minimisation, let us now focus again step by step on the four cases according to the different possible values of $K_{12}^{-1}$. In each case, we shall fix the axions at their possible VEVs and then study the Kähler moduli stabilisation depending on the particular form of $K_{11}^{-1}$ and $K_{22}^{-1}$.

However, before focusing on each particular case, let us point out some general features. At the axion minimum we will have:

$$
\left\langle V_{A X}\right\rangle=2\left( \pm\left|Y_{12}\right| \pm\left|X_{1}\right| \pm\left|X_{2}\right|\right)
$$

where the " \pm " signs depend on the specific locus of the minimum, that is (a) or (b) or (c), as specified in (A.57). Now to write (A.90) explicitly, recall (A.63) and get:

$$
V_{n p 2}+V_{n p 1}^{A X}=\frac{2}{\mathcal{V}^{2}}\left\{W \sum_{j=1}^{2}\left( \pm 2 a_{j} \tau_{j} e^{-a_{j} \tau_{j}}\right) \pm K_{12}^{-1} a_{1} a_{2} e^{-\left(a_{1} \tau_{1}+a_{2} \tau_{2}\right)}\right\}
$$


where we have set $A_{1}=A_{2}=1$. We may now study the full potential by combining equations (A.2), A.41) and (A.91)

$$
\begin{aligned}
V \sim & \frac{1}{\mathcal{V}^{2}}\left[\sum_{j=1}^{2} K_{j j}^{-1} a_{j}^{2} e^{-2 a_{j} \tau_{j}} \pm 2 K_{12}^{-1} a_{1} a_{2} e^{-\left(a_{1} \tau_{1}+a_{2} \tau_{2}\right)}\right] \\
& +4 \frac{W_{0}}{\mathcal{V}^{2}} \sum_{j=1}^{2}\left( \pm a_{j} \tau_{j} e^{-a_{j} \tau_{j}}\right)+\frac{3}{4} \frac{\hat{\xi}}{\mathcal{V}^{3}} W_{0}^{2},
\end{aligned}
$$

where we have substituted $W$ with its tree-level expectation value $W_{0}$ because the nonperturbative corrections are always subleading by a power of $\mathcal{V}$.

When we take the generic large volume limit $\mathcal{V} \sim e^{\beta a_{1} \tau_{1}} \sim e^{\gamma a_{2} \tau_{2}}$, with $\beta$ and $\gamma \in \mathbb{R}$ without any particular relation among them to take into account all the possible limits A.64), (A.92) has the following volume scaling

$$
V \sim \frac{K_{11}^{-1}}{\mathcal{V}^{2+2 / \beta}}+\frac{K_{22}^{-1}}{\mathcal{V}^{2+2 / \gamma}} \pm \frac{K_{12}^{-1}}{\mathcal{V}^{2+1 / \beta+1 / \gamma}} \pm \frac{\tau_{1}}{\mathcal{V}^{2+1 / \beta}} \pm \frac{\tau_{2}}{\mathcal{V}^{2+1 / \gamma}}+\frac{1}{\mathcal{V}^{3}}
$$

Now given that we are already aware of the volume scaling of $K_{12}^{-1}$, which was our starting point to stabilise the axions, in order to understand what are the leading terms in (A.93), we need to know only the form of $K_{j j}^{-1}, j=1,2$. We can classify its behaviour according to the volume dependence of the quantity $k_{j j k} t^{k}$ and find 4 different cases as we did for $K_{12}^{-1}$ :

1. $k_{j j k} t^{k}=0$ or $k_{j j k} t^{k}=\frac{f\left(\tau_{1}, \tau_{2}\right)}{\mathcal{V}^{\alpha}}, \alpha \geq 1 \Longrightarrow K_{j j}^{-1}=\tau_{j}^{2}$;

2. $k_{j j k} t^{k}=\frac{g_{\gamma}\left(\tau_{1}, \tau_{2}\right)}{\mathcal{V}^{\alpha}}, 0<\alpha<1, g$ homogeneous function of degree $\gamma=\frac{1+3 \alpha}{2} \Longrightarrow K_{j j}^{-1}=$ $\mathcal{V}^{\alpha} g_{2-3 \alpha / 2}\left(\tau_{1}, \tau_{2}\right), \quad 0<\alpha<1$;

3. $k_{j j k} t^{k}=f_{1 / 2}\left(\tau_{1}, \tau_{2}\right), f$ homogeneous function of degree $1 / 2 \Longrightarrow K_{j j}^{-1}=\mathcal{V} f_{1 / 2}\left(\tau_{1}, \tau_{2}\right)$;

4. $k_{j j k} t^{k}=\mathcal{V}^{\alpha} h_{\beta}\left(\tau_{1}, \tau_{2}\right), \alpha>0, h$ homogeneous function of degree $\beta=\frac{1-3 \alpha}{2} \Longrightarrow K_{j j}^{-1}=$ $\mathcal{V}^{\alpha} h_{2-3 \alpha / 2}\left(\tau_{1}, \tau_{2}\right), \quad \alpha>1$.

Before focusing on the Kähler moduli minimisation by analysing all these 4 cases in full detail for each direction (A.64), we stress that we can already show in general that some situations do not lead to any LARGE Volume minimum.

For example, let us assume that the elements of $K^{-1}$ are such that the dominant terms in A.93) are

$$
V \sim \frac{K_{11}^{-1}}{\mathcal{V}^{2+2 / \beta}}-\frac{\tau_{1}}{\mathcal{V}^{2+1 / \beta}}-\frac{\tau_{2}}{\mathcal{V}^{2+1 / \gamma}}+\frac{1}{\mathcal{V}^{3}}
$$

with $\beta=\gamma=1$ and $K_{11}^{-1}=\mathcal{V} f_{1 / 2}\left(\tau_{1}, \tau_{2}\right)$. Therefore the potential (A.92) with $W_{0}=1$ looks like

$$
V \sim \frac{f_{1 / 2}\left(\tau_{1}, \tau_{2}\right) a_{1}^{2} e^{-2 a_{1} \tau_{1}}}{\mathcal{V}}-\frac{4 a_{1} \tau_{1} e^{-a_{1} \tau_{1}}}{\mathcal{V}^{2}}-\frac{4 a_{2} \tau_{2} e^{-a_{2} \tau_{2}}}{\mathcal{V}^{2}}+\frac{3}{4} \frac{\hat{\xi}}{\mathcal{V}^{3}}
$$


Thus from $\frac{\partial V}{\partial \tau_{1}}=0$ we find

$$
\mathcal{V}=\frac{4 \tau_{1}}{\left(2 a_{1} f_{1 / 2}-\frac{\partial f_{1 / 2}}{\partial \tau_{1}}\right)} e^{a_{1} \tau_{1}},
$$

whereas $\frac{\partial V}{\partial \tau_{2}}=0$ gives

$$
\mathcal{V}=-4\left(\frac{a_{2}}{a_{1}}\right)^{2} \frac{\tau_{2}}{\frac{\partial f_{1 / 2}}{\partial \tau_{2}}} \frac{e^{2 a_{1} \tau_{1}}}{e^{a_{2} \tau_{2}}}
$$

Now since we have $\beta=\gamma=1$, from the form of the large volume limit (I) of (A.64), we infer that the minimum should be located at $a_{1} \tau_{1} \simeq a_{2} \tau_{2}$. Making this substitution and combining (A.96) with (A.97), we end up with the following equation

$$
\frac{\partial f_{1 / 2}}{\partial \tau_{2}}=\frac{a_{2}}{a_{1}} \frac{\partial f_{1 / 2}}{\partial \tau_{1}}-2 a_{2} f_{1 / 2}
$$

Now using the homogeneity property of $f_{1 / 2}$, that is $\tau_{1} \frac{\partial f_{1 / 2}}{\partial \tau_{1}}+\tau_{2} \frac{\partial f_{1 / 2}}{\partial \tau_{2}}=\frac{1}{2} f_{1 / 2}$, (A.98) takes the form

$$
\frac{\partial f_{1 / 2}}{\partial \tau_{2}}=a_{2}\left(\frac{1}{4 a_{2} \tau_{2}}-1\right) f_{1 / 2}
$$

We can solve the previous differential equation getting $f_{1 / 2}=\tau_{2}^{1 / 4} e^{-a_{2} \tau_{2}}$, which is not an homogeneous function of degree $1 / 2$. Thus we deduce that this case gives no LVS.

Another case in which we can show explicitly that no LARGE Volume minimum is present, is the one where the dominant terms in (A.93) read

$$
V \sim \frac{K_{11}^{-1}}{\mathcal{V}^{2+2 / \beta}}+\frac{K_{22}^{-1}}{\mathcal{V}^{2+2 / \gamma}}+\frac{1}{\mathcal{V}^{3}}
$$

with $\beta \leq \gamma$. The fact that all the three terms in (A.100) are strictly positive leads us to conclude that there would definitely be no LARGE Volume minimum in the volume direction once we integrate out the small moduli. In fact, (A.100) would take the generic form

$$
V \sim \frac{a(\ln \mathcal{V})^{b}+c}{\mathcal{V}^{3}}, \quad c>0
$$

which can be easily seen to have a minimum only if $a<0$ and $b>0$.

We illustrate now a further case in which it is possible to show explicitly that no LVS is present. The leading terms in (A.93) read

$$
V \sim \frac{K_{11}^{-1}}{\mathcal{V}^{2+2 / \beta}}+\frac{K_{22}^{-1}}{\mathcal{V}^{2+2 / \gamma}}-\frac{\tau_{2}}{\mathcal{V}^{2+1 / \gamma}}
$$

with $\beta<\gamma$ and $\gamma>1$ to be able to neglect the $\alpha^{\prime}$ corrections that scale as $\mathcal{V}^{-3}$. The necessary but not sufficient condition to fix the small Kähler moduli is $K_{11}^{-1}=\mathcal{V}^{\delta} \tau_{1}^{2-3 \delta / 2}$ and $K_{22}^{-1}=\mathcal{V}^{\eta} f_{2-3 \eta / 2}\left(\tau_{1}, \tau_{2}\right)$ with $\delta=2 / \beta-1 / \gamma$ and $\eta=1 / \gamma$. We can now prove that it is never possible to stabilise $a_{1} \tau_{1} \gg 1$. In fact, the relevant part of the scalar potential (A.92) would read

$$
V \simeq \frac{a_{1}^{2} \tau_{1}^{2-3 \delta / 2} e^{-2 a_{1} \tau_{1}}}{\mathcal{V}^{2-\delta}}+\frac{a_{2}^{2} f_{2-3 \eta / 2}\left(\tau_{1}, \tau_{2}\right) e^{-2 a_{2} \tau_{2}}}{\mathcal{V}^{2-\eta}}-\frac{4 a_{2} \tau_{2} e^{-a_{2} \tau_{2}}}{\mathcal{V}^{2}}
$$


Now the equation $\frac{\partial V}{\partial \tau_{1}}=0$, admits a solution of the form

$$
\mathcal{V}^{\eta-\delta}=\frac{2 a_{1}^{3} \tau_{1}^{2-3 \delta / 2}}{a_{2}^{2} \frac{\partial f_{2-3 \eta / 2}}{\partial \tau_{1}}} e^{2\left(a_{2} \tau_{2}-a_{1} \tau_{1}\right)}
$$

whereas $\frac{\partial V}{\partial \tau_{2}}=0$ gives

$$
\mathcal{V}^{\eta}=\frac{2 \tau_{2}}{a_{2} f_{2-3 \eta / 2}} e^{a_{2} \tau_{2}}
$$

The third minimisation equation $\frac{\partial V}{\partial \mathcal{V}}=0$ looks like

$$
(\eta-2) a_{2}^{2} f_{2-3 \eta / 2} \mathcal{V}^{\eta} e^{-2 a_{2} \tau_{2}}+(\delta-2) a_{1}^{2} \tau_{1}^{2-3 \delta / 2} \mathcal{V}^{\delta} e^{-2 a_{1} \tau_{1}}+8 a_{2} \tau_{2} e^{-a_{2} \tau_{2}}=0,
$$

and substituting the results (A.105) and (A.104), we obtain

$$
2(\eta-2)+\frac{(\delta-2)}{a_{1} f_{2-3 \eta / 2}} \frac{\partial f_{2-3 \eta / 2}}{\partial \tau_{1}}+8=0 .
$$

Solving the differential equation (A.107), we realise that $f_{2-3 \eta / 2}$ has an exponential behaviour in $\tau_{1}$ which is in clear contrast with the requirement that it has to be homogeneous. Following arguments very similar to this one it can be seen that, as in the case with just one small modulus, the presence of the $\alpha^{\prime}$ corrections is crucial to find a LARGE Volume minimum. In fact if we omit them, either it is impossible to fix the small moduli large enough to ignore higher instanton corrections or, once we integrate them out, we are left with a run-away in the volume direction.

Lastly, we describe the final case in which it is possible to prove the absence of a LARGE Volume vacuum. The leading terms in (A.93) are given by

$$
V \sim \frac{K_{11}^{-1}}{\mathcal{V}^{2+2 / \beta}}+\frac{K_{22}^{-1}}{\mathcal{V}^{4}}-\frac{\tau_{2}}{\mathcal{V}^{3}}+\frac{1}{\mathcal{V}^{3}}
$$

with $\beta<1$ and the axion minimum along the direction $\mathcal{V} \sim e^{\beta a_{1} \tau_{1}} \sim e^{a_{2} \tau_{2}}$. The necessary but not sufficient condition to fix the small Kähler moduli is $K_{11}^{-1} \simeq \mathcal{V}^{\delta} \tau_{1}^{2-3 \delta / 2}$ with $\delta=$ $2 / \beta-1$, and $K_{22}^{-1}=\mathcal{V} f_{1 / 2}\left(\tau_{1}, \tau_{2}\right)$. The relevant part of the scalar potential (A.92) takes the form

$$
V \simeq \frac{a_{1}^{2} \tau_{1}^{2-3 \delta / 2} e^{-2 a_{1} \tau_{1}}}{\mathcal{V}^{2-\delta}}+\frac{a_{2}^{2} f_{1 / 2}\left(\tau_{1}, \tau_{2}\right) e^{-2 a_{2} \tau_{2}}}{\mathcal{V}}-\frac{4 a_{2} \tau_{2} e^{-a_{2} \tau_{2}}}{\mathcal{V}^{2}}+\frac{3}{4} \frac{\hat{\xi}}{\mathcal{V}^{3}}
$$

Now the equation $\frac{\partial V}{\partial \tau_{1}}=0$, admits a solution of the form

$$
a_{1}^{2} \tau_{1}^{2-3 \delta / 2} \mathcal{V}^{\delta} e^{-2 a_{1} \tau_{1}}=\frac{a_{2}^{2}}{2 a_{1}} \frac{\partial f_{1 / 2}}{\partial \tau_{1}} \mathcal{V} e^{-2 a_{2} \tau_{2}},
$$

whereas $\frac{\partial V}{\partial \tau_{2}}=0$ gives

$$
a_{2} \tau_{2} e^{-a_{2} \tau_{2}}=\frac{a_{2}^{2}}{2} f_{1 / 2} \mathcal{V} e^{-2 a_{2} \tau_{2}}
$$


The third minimisation equation $\frac{\partial V}{\partial \mathcal{V}}=0$ corresponds to

$$
(\delta-2) a_{1}^{2} \tau_{1}^{2-3 \delta / 2} \mathcal{V}^{\delta} e^{-2 a_{1} \tau_{1}}-a_{2}^{2} f_{1 / 2} \mathcal{V} e^{-2 a_{2} \tau_{2}}-8 a_{2} \tau_{2} e^{-a_{2} \tau_{2}}=\frac{9}{4} \frac{\hat{\xi}}{\mathcal{V}}
$$

and substituting the results (A.110) and (A.111), we obtain

$$
4 a_{2}^{2} \mathcal{V}^{2}\left[\frac{(\delta-2)}{2 a_{1}} \frac{\partial f_{1 / 2}}{\partial \tau_{1}}-5 f_{1 / 2}\right]=9 \hat{\xi} e^{2 a_{2} \tau_{2}}
$$

Now writing $f_{1 / 2}\left(\tau_{1}, \tau_{2}\right)=F\left(\frac{\beta a_{1}}{a_{2}}\right) \sqrt{\tau_{1}}$ for appropriate function $F$, A.113 becomes

$$
\frac{a_{2}^{2}}{a_{1} \sqrt{\tau_{1}}} \mathcal{V}^{2} F\left(\frac{\beta a_{1}}{a_{2}}\right)\left(\delta-2-20 a_{1} \tau_{1}\right)=9 \hat{\xi} e^{2 a_{2} \tau_{2}} \text {. }
$$

Given that a trustable minimum requires $a_{1} \tau_{1} \gg 1$, the LHS of (A.114) is negative while the RHS is definitively positive and so this case does not allow us to find any LVS.

The general path that we shall follow to derive the conditions which guarantee that we have enough terms with the correct volume scaling to stabilise all the moduli at exponentially large volume, is the following one. We learnt from the proof of Claim 1 for the case with just one small modulus $\tau_{s}$, that we need to have two terms in the scalar potential with the same volume scaling that depend on $\tau_{s}$ so that it can be stabilised rather large in order to be able to neglect higher instanton corrections. Then if we integrate out $\tau_{s}$, we have to be left with at least two terms that depend on the overall volume and have the same volume scaling. Lastly in order to find the exponentially large volume minimum, the leading term at large volume has to be negative. As we have seen before, the same arguments apply here. Thus we shall first work out the conditions to be able to fix both $a_{1} \tau_{1} \gg 1$ and $a_{2} \tau_{2} \gg 1$ by having at least two terms in the potential with a dependence on these moduli and the same volume scaling. Then, we shall imagine to integrate out these moduli, and derive the conditions to be left with at least two terms dependent on $\mathcal{V}$ with the leading one which is negative.

\section{Case (1): $K_{12}^{-1} \simeq \tau_{1} \tau_{2}$}

The previous analysis tells us that, regardless of the particular direction considered, the axion minimum is always in the case (b). Thus we realise that at the minimum

$$
\left\langle V_{A X}\right\rangle=2\left(\left|Y_{12}\right|-\left|X_{1}\right|-\left|X_{2}\right|\right) .
$$

Now recalling that in case $(1) K_{12}^{-1} \simeq \tau_{1} \tau_{2}$, (A.92) takes the form:

$$
\begin{aligned}
V \sim & \frac{1}{\mathcal{V}^{2}}\left[\sum_{j=1}^{2} K_{j j}^{-1} a_{j}^{2} e^{-2 a_{j} \tau_{j}}+2 \tau_{1} \tau_{2} a_{1} a_{2} e^{-\left(a_{1} \tau_{1}+a_{2} \tau_{2}\right)}\right] \\
& -4 \frac{W_{0}}{\mathcal{V}^{2}} \sum_{j=1}^{2} a_{j} \tau_{j} e^{-a_{j} \tau_{j}}+\frac{3}{4} \frac{\hat{\xi}}{\mathcal{V}^{3}} W_{0}^{2} .
\end{aligned}
$$


We shall now study the behaviour of (A.116) by taking the large volume limit along each direction (A.64) and then considering all the possible forms of $K_{j j}^{-1}, j=1,2$. When we take the large volume limit (I) of (A.64), A.116) has the following volume scaling

$$
V \sim \frac{K_{11}^{-1}}{\mathcal{V}^{2+2 / \gamma}}+\frac{K_{22}^{-1}}{\mathcal{V}^{2+2 / \gamma}}+\frac{\tau_{1} \tau_{2}}{\mathcal{V}^{2+2 / \gamma}}-\frac{\tau_{1}}{\mathcal{V}^{2+1 / \gamma}}-\frac{\tau_{2}}{\mathcal{V}^{2+1 / \gamma}}+\frac{1}{\mathcal{V}^{3}}
$$

The third term in A.117) is subleading with respect to the fourth and the fifth. Thus it can be neglected

$$
V \sim \frac{K_{11}^{-1}}{\mathcal{V}^{2+2 / \gamma}}+\frac{K_{22}^{-1}}{\mathcal{V}^{2+2 / \gamma}}-\frac{\tau_{1}}{\mathcal{V}^{2+1 / \gamma}}-\frac{\tau_{2}}{\mathcal{V}^{2+1 / \gamma}}+\frac{1}{\mathcal{V}^{3}}
$$

We have seen that the presence of the $\alpha^{\prime}$ corrections is crucial to find the exponentially large volume minimum. Therefore the fact that the third and the fourth terms in (A.118) have to scale as $\mathcal{V}^{-3}$ tells us that $\gamma=1$.

$$
V \sim \frac{K_{11}^{-1}}{\mathcal{V}^{4}}+\frac{K_{22}^{-1}}{\mathcal{V}^{4}}-\frac{\tau_{1}}{\mathcal{V}^{3}}-\frac{\tau_{2}}{\mathcal{V}^{3}}+\frac{1}{\mathcal{V}^{3}}
$$

At this point it is straightforward to realise that if either $K_{11}^{-1}$ or $K_{22}^{-1}$ were in case (4), then we would have a run-away behaviour of the volume direction. Similarly the situation with $K_{11}^{-1}$ and $K_{22}^{-1}$ either in case (1) or (2) is not giving a LARGE Volume minimum since the first two terms in (A.119) should be neglected without then the possibility to stabilise $\tau_{1}$ and $\tau_{2}$ large. What happens if either $K_{11}^{-1}$ or $K_{22}^{-1}$ is in case (3) and the other one is either in case (1) or (2)? We do not find any minimum. In fact, let us say that $K_{11}^{-1}$ is in case (3) and $K_{22}^{-1}$ in case (1) or (2): then the second term in (A.119) can be neglected. If we want to have still some hope to stabilise $\tau_{2}$ large, then $K_{11}^{-1}$ should better depend also on $\tau_{2}: K_{11}^{-1} \simeq f_{1 / 2}\left(\tau_{1}, \tau_{2}\right) \mathcal{V}$. However this case has been studied explicitly to show that it leads to an absurd. Thus only if both $K_{11}^{-1}$ and $K_{22}^{-1}$ is in case (3) we can have a LARGE Volume minimum.

On the contrary, if we took either the large volume limit (II) or (III) in (A.64), (A.116) would scale as

$$
V \sim \frac{K_{11}^{-1}}{\mathcal{V}^{2+2 / \beta}}+\frac{K_{22}^{-1}}{\mathcal{V}^{2+2 / \gamma}}+\frac{\tau_{1} \tau_{2}}{\mathcal{V}^{2+1 / \beta+1 / \gamma}}-\frac{\tau_{1}}{\mathcal{V}^{2+1 / \beta}}-\frac{\tau_{2}}{\mathcal{V}^{2+1 / \gamma}}+\frac{1}{\mathcal{V}^{3}}
$$

Let us focus on the direction (II) where $1 / \beta>1 / \gamma$. The third and the fourth term in A.120 at large volume are subdominant to the fifth and therefore they can be ignored:

$$
V \sim \frac{K_{11}^{-1}}{\mathcal{V}^{2+2 / \beta}}+\frac{K_{22}^{-1}}{\mathcal{V}^{2+2 / \gamma}}-\frac{\tau_{2}}{\mathcal{V}^{2+1 / \gamma}}+\frac{1}{\mathcal{V}^{3}}
$$

If $1 / \gamma>1$, then the $\mathcal{V}^{-3}$ term would be the dominant one producing a run-away in the volume direction. Thus we impose $1 / \gamma \leq 1$. However we have already showed that the situation with $1 / \gamma<1$ gives no LVS and so we deduce that we need $1 / \gamma=1$. Then we realise that the only possible situation in which we can hope to fix $\tau_{2}$ large is when either the first or the second term in (A.121) scales as $\mathcal{V}^{-3}$. Now if the second term involving $K_{22}^{-1}$ 
were subleading with respect to the fourth term in (A.121), then the first one should scale as $\mathcal{V}^{-3}$. However at that point, knowing that $K_{11}^{-1}$ will introduce a dependence on $\tau_{1}$, we would not be able to stabilise $\tau_{1}$ large. Hence $K_{22}^{-1}$ has to be in case $(3): K_{22}^{-1}=\mathcal{V} f_{1 / 2}\left(\tau_{1}, \tau_{2}\right)$.

Now we have two different situations according to the fact that $f_{1 / 2}$ indeed depends on both $\tau_{1}$ and $\tau_{2}$ or only on $\tau_{2}$. The first possibility has already been studied with the final conclusion that it produces no LVS. On the other hand, when $K_{22}^{-1}$ depends only on $\tau_{2}$, i.e. $K_{22}^{-1} \simeq \mathcal{V} \sqrt{\tau_{2}}$, we have that the overall volume and $\tau_{2}$ are both stabilised by the interplay of the second, the third and the fourth term in (A.121). The first term is now subleading and can be used to fix $\tau_{1}$ if we write $K_{11}^{-1} \simeq \mathcal{V}^{\alpha} \tau_{1}^{2-3 \alpha / 2}$ and then impose $1 / \beta=\alpha$ in order to make it scale as the fourth term in (A.120).

We point out that these results apply also to the direction (III) where $1 / \gamma>1 / \beta$, if we swap $\gamma$ with $\beta$ and $\tau_{1}$ with $\tau_{2}$. Let us finally summarise in the table below what we have found for this case.

Case (1): $K_{12}^{-1} \simeq \tau_{1} \tau_{2}$

\begin{tabular}{|c|c|c|c|c|}
\hline$K_{11}^{-1}$ & $K_{22}^{-1}$ & $(\mathrm{I}),(\mathrm{b})$ & $(\mathrm{II}),(\mathrm{b})$ & $(\mathrm{III}),(\mathrm{b})$ \\
\hline 1 & 1 & $\mathrm{NO}$ & $\mathrm{NO}$ & $\mathrm{NO}$ \\
\hline 1 & 2 & $\mathrm{NO}$ & $\mathrm{NO}$ & $\mathrm{NO}$ \\
\hline 1 & 3 & $\mathrm{NO}$ & $\mathrm{NO}$ & $\mathrm{NO}$ \\
\hline 1 & 4 & $\mathrm{NO}$ & $\mathrm{NO}$ & $\mathrm{NO}$ \\
\hline 2 & 1 & $\mathrm{NO}$ & $\mathrm{NO}$ & $\mathrm{NO}$ \\
\hline 2 & 2 & $\mathrm{NO}$ & $\mathrm{NO}$ & $\mathrm{NO}$ \\
\hline 2 & 3 & $\mathrm{NO}$ & $\mathrm{NO}$ & $\mathrm{NO}$ \\
\hline 2 & 4 & $\mathrm{NO}$ & $\mathrm{NO}$ & $\mathrm{NO}$ \\
\hline 3 & 1 & $\mathrm{NO}$ & $\mathrm{NO}$ & $\mathrm{NO}$ \\
\hline 3 & 2 & $\mathrm{NO}$ & $\mathrm{NO}$ & $\mathrm{NO}$ \\
\hline 3 & 3 & $\mathrm{OK}, \gamma=1$ & $\mathrm{NO}$ & $\mathrm{NO}$ \\
\hline 3 & 4 & $\mathrm{NO}$ & $\mathrm{NO}$ & $\mathrm{OK}, \beta=1,(* *)$ \\
\hline 4 & 1 & $\mathrm{NO}$ & $\mathrm{NO}$ & $\mathrm{NO}$ \\
\hline 4 & 2 & $\mathrm{NO}$ & $\mathrm{NO}$ & $\mathrm{NO}$ \\
\hline 4 & 3 & $\mathrm{NO}$ & $\mathrm{OK}, \gamma=1,(*)$ & $\mathrm{NO}$ \\
\hline 4 & 4 & $\mathrm{NO}$ & $\mathrm{NO}$ & $\mathrm{NO}$ \\
\hline
\end{tabular}

(*) $K_{22}^{-1} \simeq \mathcal{V} \sqrt{\tau_{2}}, K_{11}^{-1} \simeq \mathcal{V}^{\alpha} \tau_{1}^{2-3 \alpha / 2}$ with $\frac{1}{\beta}=\alpha$

$(* *) K_{11}^{-1} \simeq \mathcal{V} \sqrt{\tau_{1}}, K_{22}^{-1} \simeq \mathcal{V}^{\alpha} \tau_{2}^{2-3 \alpha / 2}$ with $\frac{1}{\gamma}=\alpha$

Case (2): $K_{12}^{-1} \simeq \mathcal{V}^{\alpha} g_{2-3 \alpha / 2}\left(\tau_{1}, \tau_{2}\right), 0<\alpha<1$

This case is more involved than the previous one since, depending on the direction chosen for the large volume limit and the exact value of the parameter $\alpha$, the axion minimum can not only be in case (b), but also in (c) and (d). Let us start by considering each case in detail: 
- axion minimum at (c) along direction (II) for $\alpha=1 / \gamma<2 / 3$ or $1 / \gamma<\alpha<1$

We can easily conclude that no LVS is present given that, looking at the general volume scaling of the scalar potential (A.93), we can notice that the fifth term would be dominant with respect to the last one since we have always $1 / \gamma<1$. Thus the $\alpha^{\prime}$ correction would be negligible at large volume, so producing no LVS.

- axion minimum at (d) along direction (III) for $\alpha=1 / \beta<2 / 3$ or $1 / \beta<\alpha<1$

This situation looks like the previous one if we swap $\gamma$ with $\beta$ and $\tau_{1}$ with $\tau_{2}$, therefore we conclude that no LARGE Volume minimum will be present.

- axion minimum at (b) along direction (II) for $\alpha<1 / \gamma$ or $2 / 3<\alpha=1 / \gamma<1$

First of all we realise that the situation with $2 / 3<\alpha=1 / \gamma<1$ does not give rise to any LVS because the leading order $\alpha^{\prime}$ correction would be negligible at large volume. We shall therefore focus on the case $\alpha<1 / \gamma$. The scalar potential (A.92) takes the form

$$
\begin{aligned}
V \sim & \frac{1}{\mathcal{V}^{2}}\left[\sum_{j=1}^{2} K_{j j}^{-1} a_{j}^{2} e^{-2 a_{j} \tau_{j}}+2 a_{1} a_{2} g_{2-3 \alpha / 2}\left(\tau_{1}, \tau_{2}\right) \mathcal{V}^{\alpha} e^{-\left(a_{1} \tau_{1}+a_{2} \tau_{2}\right)}\right] \\
& -4 \frac{W_{0}}{\mathcal{V}^{2}}\left(a_{1} \tau_{1} e^{-a_{1} \tau_{1}}+a_{2} \tau_{2} e^{-a_{2} \tau_{2}}\right)+\frac{3}{4} \frac{\hat{\xi}}{\mathcal{V}^{3}} W_{0}^{2} .
\end{aligned}
$$

When we take the large volume limit (II) of (A.64), A.122 has the following volume scaling

$$
V \sim \frac{K_{11}^{-1}}{\mathcal{V}^{2+2 / \beta}}+\frac{K_{22}^{-1}}{\mathcal{V}^{2+2 / \gamma}}+\frac{g_{2-3 \alpha / 2}\left(\tau_{1}, \tau_{2}\right)}{\mathcal{V}^{2+1 / \beta+1 / \gamma-\alpha}}-\frac{\tau_{1}}{\mathcal{V}^{2+1 / \beta}}-\frac{\tau_{2}}{\mathcal{V}^{2+1 / \gamma}}+\frac{1}{\mathcal{V}^{3}}
$$

Setting $1 / \gamma=1$ and recalling that in this direction $1 / \beta>1 / \gamma$, the dominant terms in $(\mathrm{A} .123)$ become

$$
V \sim \frac{K_{11}^{-1}}{\mathcal{V}^{2+2 / \beta}}+\frac{K_{22}^{-1}}{\mathcal{V}^{4}}-\frac{\tau_{2}}{\mathcal{V}^{3}}+\frac{1}{\mathcal{V}^{3}}
$$

Now by noticing that equation (A.124) has the same form of A.121) if we set $1 / \gamma=1$, we can just repeat the same consideration made before and obtain that $K_{22}^{-1}$ has to be in case (3). Moreover if $K_{22}^{-1}$ depends on both $\tau_{1}$ and $\tau_{2}$, then there is no LARGE Volume minimum, but when $K_{22}^{-1}$ depends only on $\tau_{2}$, the first term in (A.124) is negligible at large volume and can be used to fix $\tau_{1}$ if we make it compete with the fourth term in (A.123) by writing $K_{11}^{-1} \simeq \mathcal{V}^{\delta} \tau_{1}^{2-3 \delta / 2}$ and then imposing $1 / \beta=\delta$.

- axion minimum at (b) along direction (III) for $\alpha<1 / \beta$ or $2 / 3<\alpha=1 / \beta<1$

This situation looks like the previous one if we swap $\gamma$ with $\beta$ and $\tau_{1}$ with $\tau_{2}$, therefore we do not need to discuss this case.

- axion minimum at (b) along direction (I) for $\alpha<1 / \gamma$ or $2 / 3<\alpha=1 / \gamma<1$ 
In this situation the full scalar potential still looks like (A.122), but the volume scaling behaviour of its terms now reads

$$
V \sim \frac{K_{11}^{-1}}{\mathcal{V}^{2+2 / \gamma}}+\frac{K_{22}^{-1}}{\mathcal{V}^{2+2 / \gamma}}+\frac{g_{2-3 \alpha / 2}\left(\tau_{1}, \tau_{2}\right)}{\mathcal{V}^{2+2 / \gamma-\alpha}}-\frac{\tau_{1}}{\mathcal{V}^{2+1 / \gamma}}-\frac{\tau_{2}}{\mathcal{V}^{2+1 / \gamma}}+\frac{1}{\mathcal{V}^{3}}
$$

For $2 / 3<\alpha=1 / \gamma<1$ the last term in (A.125) would be subdominant with respect to the fifth one, but we know that its presence is crucial to find a minimum and so we can conclude that this case admits no minimum. On the other hand for $\alpha<1 / \gamma$, setting $1 / \gamma=1$, the dominant terms in A.125 become

$$
V \sim \frac{K_{11}^{-1}}{\mathcal{V}^{4}}+\frac{K_{22}^{-1}}{\mathcal{V}^{4}}-\frac{\tau_{1}}{\mathcal{V}^{3}}-\frac{\tau_{2}}{\mathcal{V}^{3}}+\frac{1}{\mathcal{V}^{3}}
$$

We immediately realise that (A.126) is absolutely similar to (A.119). We can therefore repeat exactly the same analysis and conclude that only if both $K_{11}^{-1}$ and $K_{22}^{-1}$ is in case (3) we can have a LARGE volume minimum. Let us finally summarise in the table below what we have found for this case.

Case (2): $K_{12}^{-1}=\mathcal{V}^{\alpha} g_{2-3 \alpha / 2}\left(\tau_{1}, \tau_{2}\right), 0<\alpha<1$

\begin{tabular}{|c|c|c|c|c|c|c|}
\hline$K_{11}^{-1}$ & $K_{22}^{-1}$ & $\begin{array}{c}(I),(b), \\
\alpha \leq 1 / \gamma\end{array}$ & $\begin{array}{c}(I I),(b), \\
\alpha \leq 1 / \gamma\end{array}$ & $\begin{array}{c}(I I),(c), \\
\alpha \geq 1 / \gamma\end{array}$ & $\begin{array}{c}(I I I),(b), \\
\alpha \leq 1 / \beta\end{array}$ & $\begin{array}{c}(I I I),(d), \\
\alpha \geq 1 / \beta\end{array}$ \\
\hline 1 & 1 & $\mathrm{NO}$ & $\mathrm{NO}$ & $\mathrm{NO}$ & $\mathrm{NO}$ & $\mathrm{NO}$ \\
\hline 1 & 2 & $\mathrm{NO}$ & $\mathrm{NO}$ & $\mathrm{NO}$ & $\mathrm{NO}$ & $\mathrm{NO}$ \\
\hline 1 & 3 & $\mathrm{NO}$ & $\mathrm{NO}$ & $\mathrm{NO}$ & $\mathrm{NO}$ & $\mathrm{NO}$ \\
\hline 1 & 4 & $\mathrm{NO}$ & $\mathrm{NO}$ & $\mathrm{NO}$ & $\mathrm{NO}$ & $\mathrm{NO}$ \\
\hline 2 & 1 & $\mathrm{NO}$ & $\mathrm{NO}$ & $\mathrm{NO}$ & $\mathrm{NO}$ & $\mathrm{NO}$ \\
\hline 2 & 2 & $\mathrm{NO}$ & $\mathrm{NO}$ & $\mathrm{NO}$ & $\mathrm{NO}$ & $\mathrm{NO}$ \\
\hline 2 & 3 & $\mathrm{NO}$ & $\mathrm{NO}$ & $\mathrm{NO}$ & $\mathrm{NO}$ & $\mathrm{NO}$ \\
\hline 2 & 4 & $\mathrm{NO}$ & $\mathrm{NO}$ & $\mathrm{NO}$ & $\mathrm{NO}$ & $\mathrm{NO}$ \\
\hline 3 & 1 & $\mathrm{NO}$ & $\mathrm{NO}$ & $\mathrm{NO}$ & $\mathrm{NO}$ & $\mathrm{NO}$ \\
\hline 3 & 2 & $\mathrm{NO}$ & $\mathrm{NO}$ & $\mathrm{NO}$ & $\mathrm{NO}$ & $\mathrm{NO}$ \\
\hline 3 & 3 & $\mathrm{OK}, \gamma=1$ & $\mathrm{NO}$ & $\mathrm{NO}$ & $\mathrm{NO}$ & $\mathrm{NO}$ \\
\hline 3 & 4 & $\mathrm{NO}$ & $\mathrm{NO}$ & $\mathrm{NO}$ & $\mathrm{OK}, \beta=1(* *)$ & $\mathrm{NO}$ \\
\hline 4 & 1 & $\mathrm{NO}$ & $\mathrm{NO}$ & $\mathrm{NO}$ & $\mathrm{NO}$ & $\mathrm{NO}$ \\
\hline 4 & 2 & $\mathrm{NO}$ & $\mathrm{NO}$ & $\mathrm{NO}$ & $\mathrm{NO}$ & $\mathrm{NO}$ \\
\hline 4 & 3 & $\mathrm{NO}$ & $\mathrm{OK}, \gamma=1(*)$ & $\mathrm{NO}$ & $\mathrm{NO}$ & $\mathrm{NO}$ \\
\hline 4 & 4 & $\mathrm{NO}$ & $\mathrm{NO}$ & $\mathrm{NO}$ & $\mathrm{NO}$ & $\mathrm{NO}$ \\
\hline
\end{tabular}

$(*) \quad K_{22}^{-1} \simeq \mathcal{V} \sqrt{\tau_{2}}, K_{11}^{-1} \simeq \mathcal{V}^{\delta} \tau_{1}^{2-3 \delta / 2}$ with $\frac{1}{\beta}=\delta$

$(* *) K_{11}^{-1} \simeq \mathcal{V} \sqrt{\tau_{1}}, K_{22}^{-1} \simeq \mathcal{V}^{\delta} \tau_{2}^{2-3 \delta / 2}$ with $\frac{1}{\gamma}=\delta$

Case (3): $K_{12}^{-1}=\mathcal{V} f_{1 / 2}\left(\tau_{1}, \tau_{2}\right)$

We shall now consider each particular situation according to the different possible locations of the axion minimum: 
- axion minimum at (c) along direction (II) for $\gamma>1$

Thus at the minimum

$$
\left\langle V_{A X}\right\rangle=2\left(\left|X_{1}\right|-\left|Y_{12}\right|-\left|X_{2}\right|\right) .
$$

Therefore the full potential (A.92) looks like

$$
\begin{aligned}
V \sim & \frac{1}{\mathcal{V}^{2}}\left[\sum_{j=1}^{2} K_{j j}^{-1} a_{j}^{2} e^{-2 a_{j} \tau_{j}}-2 a_{1} a_{2} f_{1 / 2}\left(\tau_{1}, \tau_{2}\right) \mathcal{V} e^{-\left(a_{1} \tau_{1}+a_{2} \tau_{2}\right)}\right] \\
& +4 \frac{W_{0}}{\mathcal{V}^{2}}\left(a_{1} \tau_{1} e^{-a_{1} \tau_{1}}-a_{2} \tau_{2} e^{-a_{2} \tau_{2}}\right)+\frac{3}{4} \frac{\hat{\xi}}{\mathcal{V}^{3}} W_{0}^{2} .
\end{aligned}
$$

When we take the large volume limit (II) of (A.64), A.128) has the following volume scaling

$$
V \sim \frac{K_{11}^{-1}}{\mathcal{V}^{2+2 / \beta}}+\frac{K_{22}^{-1}}{\mathcal{V}^{2+2 / \gamma}}-\frac{f_{1 / 2}\left(\tau_{1}, \tau_{2}\right)}{\mathcal{V}^{1+1 / \beta+1 / \gamma}}+\frac{\tau_{1}}{\mathcal{V}^{2+1 / \beta}}-\frac{\tau_{2}}{\mathcal{V}^{2+1 / \gamma}}+\frac{1}{\mathcal{V}^{3}}
$$

Given that $1 / \gamma<1$, the leading order $\alpha^{\prime}$ correction would be subleading in a Large Volume limit. However, we know that its presence is crucial to find the minimum and so we conclude that this case does not present any new Large Volume vacuum.

- axion minimum at (d) along direction (III) for $\beta>1$

This situation looks like the previous one if we swap $\gamma$ with $\beta$ and $\tau_{1}$ with $\tau_{2}$, therefore we conclude that no LARGE Volume minimum is present.

- axion minimum at (b) along direction (I) for $0<\gamma \leq 1$

Thus at the minimum

$$
\left\langle V_{A X}\right\rangle=2\left(\left|Y_{12}\right|-\left|X_{1}\right|-\left|X_{2}\right|\right),
$$

and so the full potential (A.92) becomes (setting $W_{0}=1$ )

$$
\begin{aligned}
V \sim & \frac{1}{\mathcal{V}^{2}}\left[\sum_{j=1}^{2} K_{j j}^{-1} a_{j}^{2} e^{-2 a_{j} \tau_{j}}+2 a_{1} a_{2} f_{1 / 2}\left(\tau_{1}, \tau_{2}\right) \mathcal{V} e^{-\left(a_{1} \tau_{1}+a_{2} \tau_{2}\right)}\right] \\
& -\frac{4}{\mathcal{V}^{2}}\left(a_{1} \tau_{1} e^{-a_{1} \tau_{1}}+a_{2} \tau_{2} e^{-a_{2} \tau_{2}}\right)+\frac{3}{4} \frac{\hat{\xi}}{\mathcal{V}^{3}} .
\end{aligned}
$$

When we take the large volume limit (I) of (A.64), (A.131) has the following volume scaling

$$
V \sim \frac{K_{11}^{-1}}{\mathcal{V}^{2+2 / \gamma}}+\frac{K_{22}^{-1}}{\mathcal{V}^{2+2 / \gamma}}+\frac{f_{1 / 2}\left(\tau_{1}, \tau_{2}\right)}{\mathcal{V}^{1+2 / \gamma}}-\frac{\tau_{1}}{\mathcal{V}^{2+1 / \gamma}}-\frac{\tau_{2}}{\mathcal{V}^{2+1 / \gamma}}+\frac{1}{\mathcal{V}^{3}}
$$

In the case where $1 / \gamma>1$, the leading part of (A.132) at Large Volume takes the form

$$
V \sim \frac{K_{11}^{-1}}{\mathcal{V}^{2+2 / \gamma}}+\frac{K_{22}^{-1}}{\mathcal{V}^{2+2 / \gamma}}+\frac{1}{\mathcal{V}^{3}}
$$


We have already checked explicitly that this situation does not present any LARGE Volume minimum, therefore we shall focus only on the case $1 / \gamma=1$, for which the volume scaling A.132 becomes

$$
V \sim \frac{K_{11}^{-1}}{\mathcal{V}^{4}}+\frac{K_{22}^{-1}}{\mathcal{V}^{4}}+\frac{f_{1 / 2}\left(\tau_{1}, \tau_{2}\right)}{\mathcal{V}^{3}}-\frac{\tau_{1}}{\mathcal{V}^{3}}-\frac{\tau_{2}}{\mathcal{V}^{3}}+\frac{1}{\mathcal{V}^{3}}
$$

We immediately realise that as soon as either $K_{11}^{-1}$ or $K_{22}^{-1}$ is in case (4) then the $\alpha^{\prime}$ corrections would be subleading in a large volume expansion. Thus we can reject this possibility. Then we are left with three different situations. Firstly when both $K_{11}^{-1}$ and $K_{22}^{-1}$ are subdominant with respect to the last three terms in (A.134) (i.e. each $K_{j j}^{-1}$ with $j=1,2$ is either in case (1) or (2)) the scalar potential (A.131) takes the form

$$
V=\frac{2}{\mathcal{V}} a_{1} a_{2} f_{1 / 2}\left(\tau_{1}, \tau_{2}\right) e^{-\left(a_{1} \tau_{1}+a_{2} \tau_{2}\right)}-\frac{4}{\mathcal{V}^{2}}\left(a_{1} \tau_{1} e^{-a_{1} \tau_{1}}+a_{2} \tau_{2} e^{-a_{2} \tau_{2}}\right)+\frac{3}{4} \frac{\hat{\xi}}{\mathcal{V}^{3}}
$$

Since we have to find a minimum such that $a_{j} \tau_{j} \gg 1$ for $j=1,2$, we can work at leading order in a $\frac{1}{a_{1} \tau_{1}}$ and $\frac{1}{a_{2} \tau_{2}}$ expansion and obtain that $\frac{\partial^{2} V}{\partial \tau_{1}^{2}} \simeq 0$ due to the presence of just one exponential in $\tau_{1}$ in both the first and the second term in A.135). In fact, if we are interested in the dependence of $V$ on just $\tau_{1}$, A.135) can be rewritten as

$$
V=c_{1} e^{-a_{1} \tau_{1}}\left(a_{2} f_{1 / 2}\left(\tau_{1}, \tau_{2}\right) e^{-a_{2} \tau_{2}}-\frac{2 \tau_{1}}{\mathcal{V}}\right)+c_{2} \equiv c_{1} e^{-a_{1} \tau_{1}} g\left(\tau_{1}\right)+c_{2},
$$

where $c_{1}$ and $c_{2}$ are constants and $g\left(\tau_{1}\right)$ is the sum of two homogeneous functions in $\tau_{1}$. Therefore at leading order in a $\frac{1}{a_{1} \tau_{1}}$ expansion, we get:

$$
\frac{\partial V}{\partial \tau_{1}} \simeq-a_{1} c_{1} e^{-a_{1} \tau_{1}} g\left(\tau_{1}\right)=0 \Leftrightarrow g\left(\tau_{1}\right)=0
$$

which implies

$$
\left.\left.\frac{\partial^{2} V}{\partial \tau_{1}^{2}}\right|_{\min } \simeq a_{1}^{2} c_{1} e^{-a_{1} \tau_{1}} g\left(\tau_{1}\right)\right|_{\min }=0 .
$$

Similarly we have $\frac{\partial^{2} V}{\partial \tau_{2}^{2}} \simeq 0$, whereas

$$
\left.\left.\frac{\partial^{2} V}{\partial \tau_{1} \partial \tau_{2}}\right|_{\min } \simeq 2 a_{1}^{2} a_{2}^{2} f_{1 / 2} e^{-a_{1} \tau_{1}} \frac{e^{-a_{2} \tau_{2}}}{\mathcal{V}}\right|_{\min } \equiv c_{3}>0 .
$$

Therefore considering $\mathcal{V}$ constant, the Hessian matrix will look like

$$
\mathcal{H} \simeq\left(\begin{array}{cc}
0 & c_{3} \\
c_{3} & 0
\end{array}\right) \Longrightarrow \operatorname{det} \mathcal{H}=-c_{3}^{2}<0
$$

so implying that we can never have a minimum.

Secondly we have to contemplate the possibility that only one of the first two terms in (A.134) is competing with the last three ones while the other is negligible. However even this case does not yield any new LVS due the asymmetry of the dependence of 
the scalar potential in $\tau_{1}$ and $\tau_{2}$ that does not allow us to stabilise the small Kähler moduli large enough. In fact, let us assume for example that $K_{11}^{-1}$ is in case (1) or (2) and hence is negligible, whereas $K_{22}^{-1} \simeq \mathcal{V} \sqrt{\tau_{2}}$. Consequently we obtain

$$
\begin{aligned}
& \frac{\partial V}{\partial \tau_{1}}=0 \Longleftrightarrow \mathcal{V}=\frac{2 \tau_{1}}{a_{2} f_{1 / 2}} e^{a_{2} \tau_{2}}, \\
& \frac{\partial V}{\partial \tau_{2}}=0 \Longleftrightarrow \mathcal{V}=\frac{2 \tau_{2}}{a_{1} f_{1 / 2} e^{-a_{1} \tau_{1}}+a_{2} \sqrt{\tau_{2}} e^{-a_{2} \tau_{2}}} .
\end{aligned}
$$

Now combining (A.141) with (A.142) we find:

$$
a_{1} \tau_{1} f_{1 / 2} e^{a_{2} \tau_{2}-a_{1} \tau_{1}}+a_{2} \tau_{1} \sqrt{\tau_{2}}=a_{2} \tau_{2} f_{1 / 2},
$$

which evaluated along the direction $a_{1} \tau_{1} \simeq a_{2} \tau_{2}$ where the axion minimum is located, becomes

$$
a_{1} \tau_{1} f_{1 / 2}+a_{2} \tau_{1} \sqrt{\tau_{2}} \simeq a_{1} \tau_{1} f_{1 / 2} \Longleftrightarrow \sqrt{a_{1} a_{2}} \tau_{1}^{3 / 2} \simeq 0
$$

which is the negative result we mentioned above. Lastly when both $K_{11}^{-1}$ and $K_{22}^{-1}$ is in case (3) all the terms in (A.134) have the same volume scaling. It can be seen that, regardless of the form of $f_{1 / 2}$, the LARGE Volume minimum is always present.

- axion minimum at (b) along direction (II) for $0<\gamma \leq 1$

In this situation the full scalar potential still looks like (A.131), but the volume scaling behaviour of its terms now reads

$$
V \sim \frac{K_{11}^{-1}}{\mathcal{V}^{2+2 / \beta}}+\frac{K_{22}^{-1}}{\mathcal{V}^{2+2 / \gamma}}+\frac{f_{1 / 2}\left(\tau_{1}, \tau_{2}\right)}{\mathcal{V}^{1+1 / \beta+1 / \gamma}}-\frac{\tau_{1}}{\mathcal{V}^{2+1 / \beta}}-\frac{\tau_{2}}{\mathcal{V}^{2+1 / \gamma}}+\frac{1}{\mathcal{V}^{3}}
$$

Given that along the direction (II) $1 / \beta>1 / \gamma$, and the axion minimum is present for $1 / \gamma \geq 1$, the dominant terms in A.145 become

$$
V \sim \frac{K_{11}^{-1}}{\mathcal{V}^{2+2 / \beta}}+\frac{K_{22}^{-1}}{\mathcal{V}^{4}}-\frac{\tau_{2}}{\mathcal{V}^{3}}+\frac{1}{\mathcal{V}^{3}}
$$

where we have already set $\gamma=1$ because, as we argued before, this is the only possible situation when we can hope to find a LARGE Volume minimum. We notice now that whenever the first two terms in (A.146) are negligible at large volume, then we have only one term in $V$ dependent on $\tau_{2}$ and so we can never obtain a minimum at $a_{2} \tau_{2} \gg 1$. This happens if $K_{22}^{-1}$ is either in case (1) or in case (2) and $K_{11}^{-1}$ is in case (1), (2), (3) or even in case (4) if its term in (A.146) still goes like $\mathcal{V}^{\alpha}, \alpha>3$. Moreover if $K_{22}^{-1}$ is in case (4) then it would beat the last two terms in (A.146) so giving no LVS.

On the other hand, when only the first term in (A.146) is negligible at large volume, we have the possibility to find a new LVS if $K_{22}^{-1}$ is in case (3) and does not depend on $\tau_{1}$, that is $K_{22}^{-1} \simeq \mathcal{V} \sqrt{\tau_{2}}$. In fact, at leading order in a large volume expansion the scalar potential looks like the one we studied for the case with just one small modulus and we know that the corresponding LARGE Volume minimum would be 
present. However we have still to fix $\tau_{1}$. If $K_{11}^{-1}$ is in case (1), (2) or (3) then the first term in A.145) is always subleading with respect to the third and the fourth one and therefore it can be neglected. We can now focus on the third and fourth term in (A.145) which have the same volume scaling. Then combining the solution of $\frac{\partial V}{\partial \tau_{1}}=0$ and $\frac{\partial V}{\partial \tau_{2}}=0$ we end up with $\tau_{1}=\tau_{2}$ which is correct if we choose $\beta a_{1}=a_{2}$, $\beta<1$. However we have still to check the sign of $\frac{\partial^{2} V}{\partial \tau_{1}^{2}}$ which turns out to be positive only if, writing $f_{1 / 2}\left(\tau_{1}, \tau_{2}\right) \sim \tau_{1}^{\alpha} \tau_{2}^{1 / 2-\alpha}$ for arbitrary $\alpha$, we have $\alpha<1$.

The situation when $K_{22}^{-1} \simeq \mathcal{V} \sqrt{\tau_{2}}$ and $K_{11}^{-1}$ is in case (4) needs to be studied more carefully. Writing $K_{11}^{-1} \simeq \mathcal{V}^{\delta} \tau_{1}^{2-3 \delta / 2}, \delta>1$, if $\delta>\frac{2}{\beta}+1$ then the first term in (A.146) beats all the other ones so giving no LVS. If $\delta=\frac{2}{\beta}+1$, then the first term in (A.146) scales as the other ones but we have already shown that this is not an interesting situation. The only way to get a LVS is to impose $\delta \leq \frac{1}{\beta}$ to make the first term in (A.145) scale as the third and fourth term or to render it subdominant with respect to them.

Finally if $K^{-1}$ is in case (4) and $K_{22}^{-1}$ is either in case (1) or (2) then we don't find any new LVS since the second term in (A.146) would be subleading with respect to the other ones so leaving just one term, the first one, which depends on $\tau_{1}$.

- axion minimum at (b) along direction (III) for $0<\gamma<\beta \leq 1$

This situation looks like the previous one if we swap $\gamma$ with $\beta$ and $\tau_{1}$ with $\tau_{2}$, therefore we do not need to discuss this case. Let us finally summarise in the table below what we have found for this case.

Case (3): $K_{12}^{-1}=\mathcal{V} f_{1 / 2}\left(\tau_{1}, \tau_{2}\right)$ 


\begin{tabular}{|c|c|c|c|c|c|c|}
\hline$K_{11}^{-1}$ & $K_{22}^{-1}$ & $\begin{array}{c}(I),(b), \\
0<\gamma \leq 1\end{array}$ & $\begin{array}{c}(I I),(b), \\
0<\gamma \leq 1\end{array}$ & $\begin{array}{c}(I I),(c), \\
\gamma>1\end{array}$ & $\begin{array}{c}(I I I),(b), \\
0<\gamma<\beta \leq 1\end{array}$ & $\begin{array}{c}(I I I),(d), \\
\gamma \geq 1\end{array}$ \\
\hline 1 & 1 & $\mathrm{NO}$ & $\mathrm{NO}$ & $\mathrm{NO}$ & $\mathrm{NO}$ & $\mathrm{NO}$ \\
\hline 1 & 2 & $\mathrm{NO}$ & $\mathrm{NO}$ & $\mathrm{NO}$ & $\mathrm{NO}$ & $\mathrm{NO}$ \\
\hline 1 & 3 & $\mathrm{NO}$ & $\mathrm{OK}, \beta<\gamma=1(*)$ & $\mathrm{NO}$ & $\mathrm{NO}$ & $\mathrm{NO}$ \\
\hline 1 & 4 & $\mathrm{NO}$ & $\mathrm{NO}$ & $\mathrm{NO}$ & $\mathrm{NO}$ & $\mathrm{NO}$ \\
\hline 2 & 1 & $\mathrm{NO}$ & $\mathrm{NO}$ & $\mathrm{NO}$ & $\mathrm{NO}$ & $\mathrm{NO}$ \\
\hline 2 & 2 & $\mathrm{NO}$ & $\mathrm{NO}$ & $\mathrm{NO}$ & $\mathrm{NO}$ & $\mathrm{NO}$ \\
\hline 2 & 3 & $\mathrm{NO}$ & $\mathrm{OK}, \beta<\gamma=1(*)$ & $\mathrm{NO}$ & $\mathrm{NO}$ & $\mathrm{NO}$ \\
\hline 2 & 4 & $\mathrm{NO}$ & $\mathrm{NO}$ & $\mathrm{NO}$ & $\mathrm{NO}$ & $\mathrm{NO}$ \\
\hline 3 & 1 & $\mathrm{NO}$ & $\mathrm{NO}$ & $\mathrm{NO}$ & $\mathrm{OK}, \gamma<\beta=1(\star)$ & $\mathrm{NO}$ \\
\hline 3 & 2 & $\mathrm{NO}$ & $\mathrm{NO}$ & $\mathrm{NO}$ & $\mathrm{OK}, \gamma<\beta=1(\star)$ & $\mathrm{NO}$ \\
\hline 3 & 3 & $\mathrm{OK}, \gamma=1$ & $\mathrm{OK}, \beta<\gamma=1(*)$ & $\mathrm{NO}$ & $\mathrm{OK}, \gamma<\beta=1(\star)$ & $\mathrm{NO}$ \\
\hline 3 & 4 & $\mathrm{NO}$ & $\mathrm{NO}$ & $\mathrm{NO}$ & $\mathrm{OK}, \gamma<\beta=1(\star \star)$ & $\mathrm{NO}$ \\
\hline 4 & 1 & $\mathrm{NO}$ & $\mathrm{NO}$ & $\mathrm{NO}$ & $\mathrm{NO}$ & $\mathrm{NO}$ \\
\hline 4 & 2 & $\mathrm{NO}$ & $\mathrm{NO}$ & $\mathrm{NO}$ & $\mathrm{NO}$ & $\mathrm{NO}$ \\
\hline 4 & 3 & $\mathrm{NO}$ & $\mathrm{OK}, \beta<\gamma=1(* *)$ & $\mathrm{NO}$ & $\mathrm{NO}$ & $\mathrm{NO}$ \\
\hline 4 & 4 & $\mathrm{NO}$ & $\mathrm{NO}$ & $\mathrm{NO}$ & $\mathrm{NO}$ & $\mathrm{NO}$ \\
\hline
\end{tabular}

$(*) K_{22}^{-1} \simeq \mathcal{V} \sqrt{\tau_{2}}, f_{1 / 2} \sim \tau_{1}^{\alpha} \tau_{2}^{1 / 2-\alpha}$ with $\alpha<1$

$(* *) K_{22}^{-1} \simeq \mathcal{V} \sqrt{\tau_{2}}, K_{11}^{-1} \simeq \mathcal{V}^{\delta} \tau_{1}^{2-3 \delta / 2}$ and $f_{1 / 2} \sim \tau_{1}^{\alpha} \tau_{2}^{1 / 2-\alpha}$ with $\alpha<1$ for $1<\delta<\frac{1}{\beta}$ and $\forall \alpha$ for $\delta=\frac{1}{\beta}$

$(\star) K_{11}^{-1} \simeq \mathcal{V} \sqrt{\tau_{1}}, f_{1 / 2} \sim \tau_{2}^{\alpha} \tau_{1}^{1 / 2-\alpha}$ with $\alpha<1$

$(\star \star) K_{11}^{-1} \simeq \mathcal{V} \sqrt{\tau_{1}}, K_{22}^{-1} \simeq \mathcal{V}^{\delta} \tau_{2}^{2-3 \delta / 2}$ and $f_{1 / 2} \sim \tau_{2}^{\alpha} \tau_{1}^{1 / 2-\alpha}$ with $\alpha<1$ for $1<\delta<\frac{1}{\gamma}$ and $\forall \alpha$ for $\delta=\frac{1}{\gamma}$

Case (4): $K_{12}^{-1} \simeq \mathcal{V}^{\alpha} h_{2-3 \alpha / 2}\left(\tau_{1}, \tau_{2}\right), \alpha>1$

Let us focus on each particular situation according to the different possible positions of the axion minimum:

- axion minimum at (c) along direction (II) for $\alpha>1 / \gamma$

Thus at the minimum

$$
\left\langle V_{A X}\right\rangle=2\left(\left|X_{1}\right|-\left|Y_{12}\right|-\left|X_{2}\right|\right) .
$$

Therefore the full scalar potential (A.92) reads

$$
\begin{aligned}
V \sim & \frac{1}{\mathcal{V}^{2}}\left[\sum_{j=1}^{2} K_{j j}^{-1} a_{j}^{2} e^{-2 a_{j} \tau_{j}}-2 a_{1} a_{2} h_{2-3 \alpha / 2}\left(\tau_{1}, \tau_{2}\right) \mathcal{V}^{\alpha} e^{-\left(a_{1} \tau_{1}+a_{2} \tau_{2}\right)}\right] \\
& +4 \frac{W_{0}}{\mathcal{V}^{2}}\left(a_{1} \tau_{1} e^{-a_{1} \tau_{1}}-a_{2} \tau_{2} e^{-a_{2} \tau_{2}}\right)+\frac{3}{4} \frac{\hat{\xi}}{\mathcal{V}^{3}} W_{0}^{2} .
\end{aligned}
$$


When we take the large volume limit (II) of A.64), A.148 has the following volume scaling

$$
V \sim \frac{K_{11}^{-1}}{\mathcal{V}^{2+2 / \beta}}+\frac{K_{22}^{-1}}{\mathcal{V}^{2+2 / \gamma}}-\frac{h_{2-3 \alpha / 2}\left(\tau_{1}, \tau_{2}\right)}{\mathcal{V}^{2+1 / \beta+1 / \gamma-\alpha}}+\frac{\tau_{1}}{\mathcal{V}^{2+1 / \beta}}-\frac{\tau_{2}}{\mathcal{V}^{2+1 / \gamma}}+\frac{1}{\mathcal{V}^{3}}
$$

Recalling that in this direction $1 / \beta>1 / \gamma$, the dominant terms in (A.149) are

$$
V \sim \frac{K_{11}^{-1}}{\mathcal{V}^{2+2 / \beta}}+\frac{K_{22}^{-1}}{\mathcal{V}^{2+2 / \gamma}}-\frac{h_{2-3 \alpha / 2}\left(\tau_{1}, \tau_{2}\right)}{\mathcal{V}^{2+1 / \beta+1 / \gamma-\alpha}}-\frac{\tau_{2}}{\mathcal{V}^{2+1 / \gamma}}+\frac{1}{\mathcal{V}^{3}}
$$

We know that the presence of the last term in A.150 is crucial to find the LARGE Volume minimum, so in order not to make it subleading with respect to the fourth one, we need to have $1 / \gamma \geq 1$. If $1 / \gamma=1$, the new volume scaling looks like

$$
V \sim \frac{K_{11}^{-1}}{\mathcal{V}^{2+2 / \beta}}+\frac{K_{22}^{-1}}{\mathcal{V}^{4}}-\frac{h_{2-3 \alpha / 2}\left(\tau_{1}, \tau_{2}\right)}{\mathcal{V}^{3+1 / \beta-\alpha}}-\frac{\tau_{2}}{\mathcal{V}^{3}}+\frac{1}{\mathcal{V}^{3}}
$$

with $1 / \beta \geq \alpha$ to keep the last term in (A.151). Now setting $1 / \beta=\alpha>1$, A.151) reduces to

$$
V \sim \frac{K_{11}^{-1}}{\mathcal{V}^{2+2 \alpha}}+\frac{K_{22}^{-1}}{\mathcal{V}^{4}}-\frac{h_{2-3 \alpha / 2}\left(\tau_{1}, \tau_{2}\right)}{\mathcal{V}^{3}}-\frac{\tau_{2}}{\mathcal{V}^{3}}+\frac{1}{\mathcal{V}^{3}}
$$

By studying the expression (A.152), we realise that there are only three possible situations in which the necessary but not sufficient conditions to stabilise $a_{j} \tau_{j} \gg 1$, $j=1,2$, and not to neglect the leading order $\alpha^{\prime}$ corrections, can be satisfied. The first one is when $K_{22}^{-1}$ is in case (3) and depends also on $\tau_{1}: K_{22}^{-1}=\mathcal{V} f_{1 / 2}\left(\tau_{1}, \tau_{2}\right)$. In addition, the first term in A.152 is subleading with respect to the other ones given that $K_{11}^{-1}$ is in case (1) or (2) or (3) or even in case (4) but still being subleading. However we can again show that this case does not lead to any new LVS by noticing that $\partial V / \partial \tau_{1}=0$ combined with $\partial V / \partial \tau_{2}=0$ gives rise to a differential equation for $f_{1 / 2}$ whose solution is not homogeneous.

The second situations takes place when the second term in A.152 is subleading with respect to the others. This occurs when $K_{22}^{-1}$ is either in case (1) or (2) and $K_{11}^{-1}$ is in case (4). Moreover if $K_{11}^{-1} \simeq \mathcal{V}^{\delta} \tau_{1}^{2-3 \delta / 2}$ we have to impose $\delta=2 \alpha-1$. However this case would not work because the minimisation equation $\frac{\partial V}{\partial \tau_{2}}=0$ would produce a negative volume:

$$
\mathcal{V}^{\alpha}=-\frac{2 \tau_{2}}{a_{1} h_{2-3 \alpha / 2}} e^{a_{1} \tau_{1}}
$$

Finally we have to contemplate the possibility that all the terms in (A.152) have the same volume scaling. This can happen only if $K_{22}^{-1} \simeq \mathcal{V} \sqrt{\tau_{2}}$ and $K_{22}^{-1} \simeq \mathcal{V}^{\delta} \tau_{1}^{2-3 \delta / 2}$ with $\delta=(2 \alpha-1)>1$. Even this case can be seen to produce no LVS. In fact, it is possible to integrate out the overall volume from one of the usual minimisation equations, so being left with two equations in $\tau_{1}$ and $\tau_{2}$. Then given that we know that we are looking for a minimum located at $\beta a_{1} \tau_{1} \simeq a_{2} \tau_{2}$, making this substitution, we end up with two equations in just $\tau_{1}$ which can be seen to disagree. 
On the other hand, for $1 / \beta>\alpha$, we would be left with

$$
V \sim \frac{K_{11}^{-1}}{\mathcal{V}^{2+2 / \beta}}+\frac{K_{22}^{-1}}{\mathcal{V}^{4}}-\frac{\tau_{2}}{\mathcal{V}^{3}}+\frac{1}{\mathcal{V}^{3}}
$$

Now by noticing that equation (A.154) has the same form of (A.121) if we set $1 / \gamma=1$, we can just repeat the same consideration made before and obtain that $K_{22}^{-1}$ has to be in case (3). Moreover if $K_{22}^{-1}$ depends on both $\tau_{1}$ and $\tau_{2}$, then we have no LARGE Volume minimum. On the other hand, when $K_{22}^{-1}$ depends only on $\tau_{2}$, the first term in (A.154) is now negligible at large volume and can be used to fix $\tau_{1}$ if we make it compete with the third term in A.151) by writing $K_{11}^{-1} \simeq \mathcal{V}^{\delta} \tau_{1}^{2-3 \delta / 2}$ and then imposing $1 / \beta+\alpha=1+\delta$.

On the contrary, if $1 / \gamma>1$, A.150 takes the form

$$
V \sim \frac{K_{11}^{-1}}{\mathcal{V}^{2+2 / \beta}}+\frac{K_{22}^{-1}}{\mathcal{V}^{2+2 / \gamma}}-\frac{h_{2-3 \alpha / 2}\left(\tau_{1}, \tau_{2}\right)}{\mathcal{V}^{2+1 / \beta+1 / \gamma-\alpha}}+\frac{1}{\mathcal{V}^{3}}
$$

Now for $(1 / \beta+1 / \gamma-\alpha)>1$, A.155 at leading order in a large volume expansion, reduces to

$$
V \sim \frac{K_{11}^{-1}}{\mathcal{V}^{2+2 / \beta}}+\frac{K_{22}^{-1}}{\mathcal{V}^{2+2 / \gamma}}+\frac{1}{\mathcal{V}^{3}}
$$

which has already been proved to produce no LVS. On the other hand, for $(1 / \beta+$ $1 / \gamma-\alpha)<1$, the $\alpha^{\prime}$ correction would be negligible at large volume, so forcing us to impose $(1 / \beta+1 / \gamma-\alpha)=1$ and obtain:

$$
V \sim \frac{K_{11}^{-1}}{\mathcal{V}^{2+2 / \beta}}+\frac{K_{22}^{-1}}{\mathcal{V}^{2+2 / \gamma}}-\frac{h_{2-3 \alpha / 2}\left(\tau_{1}, \tau_{2}\right)}{\mathcal{V}^{3}}+\frac{1}{\mathcal{V}^{3}}
$$

However we can show explicitly that there is no LARGE Volume minimum. In fact, setting $K_{11}^{-1} \simeq \mathcal{V}^{\eta} \tau_{1}^{2-3 \eta / 2}$ with $\eta=\frac{2}{\beta}-1$, and $K_{22}^{-1} \simeq \mathcal{V}^{\delta} \tau_{2}^{2-3 \delta / 2}$ with $\delta=\frac{2}{\gamma}-1$, and then substituting the solutions of $\partial V / \partial \tau_{1}=0$ and $\partial V / \partial \tau_{2}=0$ in $\partial V / \partial \mathcal{V}=0$, one finds that it is never possible to fix the small Kähler moduli large enough to be able to neglect the higher order instanton contributions to $W$. If $h_{2-3 \alpha / 2}$ did not depend on both $\tau_{1}$ and $\tau_{2}$ but just on one of them, this negative result would not be altered as the term involving $h_{2-3 \alpha / 2}$ depends always on both the two small moduli via the two exponentials $e^{a_{1} \tau_{1}} e^{a_{2} \tau_{2}}($ see A.148) ).

- axion minimum at (d) along direction (III) for $\alpha>1 / \beta$

This situation looks like the previous one if we swap $\gamma$ with $\beta$ and $\tau_{1}$ with $\tau_{2}$, therefore we do not need to discuss this case.

- axion minimum at (b) along direction (I) for $1<\alpha \leq 1 / \gamma$

Thus at the minimum

$$
\left\langle V_{A X}\right\rangle=2\left(\left|Y_{12}\right|-\left|X_{1}\right|-\left|X_{2}\right|\right)
$$


and so the full potential A.92) becomes

$$
\begin{aligned}
V \sim & \frac{1}{\mathcal{V}^{2}}\left[\sum_{j=1}^{2} K_{j j}^{-1} a_{j}^{2} e^{-2 a_{j} \tau_{j}}+2 a_{1} a_{2} h_{2-3 \alpha / 2}\left(\tau_{1}, \tau_{2}\right) \mathcal{V}^{\alpha} e^{-\left(a_{1} \tau_{1}+a_{2} \tau_{2}\right)}\right] \\
& -4 \frac{W_{0}}{\mathcal{V}^{2}}\left(a_{1} \tau_{1} e^{-a_{1} \tau_{1}}+a_{2} \tau_{2} e^{-a_{2} \tau_{2}}\right)+\frac{3}{4} \frac{\hat{\xi}}{\mathcal{V}^{3}} W_{0}^{2} .
\end{aligned}
$$

When we take the large volume limit (I) of (A.64), (A.159) has the following volume scaling

$$
V \sim \frac{K_{11}^{-1}}{\mathcal{V}^{2+2 / \gamma}}+\frac{K_{22}^{-1}}{\mathcal{V}^{2+2 / \gamma}}+\frac{h_{2-3 \alpha / 2}\left(\tau_{1}, \tau_{2}\right)}{\mathcal{V}^{2+2 / \gamma-\alpha}}-\frac{\tau_{1}}{\mathcal{V}^{2+1 / \gamma}}-\frac{\tau_{2}}{\mathcal{V}^{2+1 / \gamma}}+\frac{1}{\mathcal{V}^{3}}
$$

Due to the fact that $1<\alpha \leq 1 / \gamma$, the third, the fourth and the fifth term in (A.160) are suppressed with respect to the remaining ones by an appropriate power of the volume and so we can neglect them. Thus the leading part of (A.160) takes the form

$$
V \sim \frac{K_{11}^{-1}}{\mathcal{V}^{2+2 / \gamma}}+\frac{K_{22}^{-1}}{\mathcal{V}^{2+2 / \gamma}}+\frac{1}{\mathcal{V}^{3}}
$$

We have already checked explicitly that this situation does not present any LARGE Volume minimum.

- axion minimum at (b) along direction (II) for $1<\alpha \leq 1 / \gamma$

In this situation the full scalar potential still looks like (A.159), but the volume scaling behaviour of its terms now reads

$$
V \sim \frac{K_{11}^{-1}}{\mathcal{V}^{2+2 / \beta}}+\frac{K_{22}^{-1}}{\mathcal{V}^{2+2 / \gamma}}+\frac{h_{2-3 \alpha / 2}\left(\tau_{1}, \tau_{2}\right)}{\mathcal{V}^{2+1 / \beta+1 / \gamma-\alpha}}-\frac{\tau_{1}}{\mathcal{V}^{2+1 / \beta}}-\frac{\tau_{2}}{\mathcal{V}^{2+1 / \gamma}}+\frac{1}{\mathcal{V}^{3}}
$$

Given that along the direction (II) $1 / \beta>1 / \gamma$, and the axion minimum is present for $1<\alpha \leq 1 / \gamma$, the dominant terms in (A.162) become

$$
V \sim \frac{K_{11}^{-1}}{\mathcal{V}^{2+2 / \beta}}+\frac{K_{22}^{-1}}{\mathcal{V}^{2+2 / \gamma}}+\frac{1}{\mathcal{V}^{3}}
$$

Thus we conclude that this case does not show any LVS, as we have already showed.

- axion minimum at (b) along direction (III) for $1<\alpha \leq 1 / \beta<1 / \gamma$

This situation looks like the previous one if we swap $\gamma$ with $\beta$ and $\tau_{1}$ with $\tau_{2}$, therefore we do not need to discuss this case. Let us finally summarise in the table below what we have found for this case.

Case (4): $K_{12}^{-1}=\mathcal{V}^{\alpha} h_{2-3 \alpha / 2}\left(\tau_{1}, \tau_{2}\right), \alpha>1$ 


\begin{tabular}{|c|c|c|c|c|c|c|}
\hline$K_{11}^{-1}$ & $K_{22}^{-1}$ & $\begin{array}{c}(I),(b), \\
1<\alpha \leq 1 / \gamma\end{array}$ & $\begin{array}{c}(I I),(b), \\
1<\alpha \leq 1 / \gamma\end{array}$ & $\begin{array}{c}(I I),(c), \\
\alpha>1 / \gamma\end{array}$ & $\begin{array}{c}(I I I),(b), \\
1<\alpha<1 / \beta<1 / \gamma\end{array}$ & $\begin{array}{c}(I I I),(d), \\
\alpha>1 / \beta\end{array}$ \\
\hline 1 & 1 & $\mathrm{NO}$ & $\mathrm{NO}$ & $\mathrm{NO}$ & $\mathrm{NO}$ & $\mathrm{NO}$ \\
\hline 1 & 2 & $\mathrm{NO}$ & $\mathrm{NO}$ & $\mathrm{NO}$ & $\mathrm{NO}$ & $\mathrm{NO}$ \\
\hline 1 & 3 & $\mathrm{NO}$ & $\mathrm{NO}$ & $\mathrm{NO}$ & $\mathrm{NO}$ & $\mathrm{NO}$ \\
\hline 1 & 4 & $\mathrm{NO}$ & $\mathrm{NO}$ & $\mathrm{NO}$ & $\mathrm{NO}$ & $\mathrm{NO}$ \\
\hline 2 & 1 & $\mathrm{NO}$ & $\mathrm{NO}$ & $\mathrm{NO}$ & $\mathrm{NO}$ & $\mathrm{NO}$ \\
\hline 2 & 2 & $\mathrm{NO}$ & $\mathrm{NO}$ & $\mathrm{NO}$ & $\mathrm{NO}$ & $\mathrm{NO}$ \\
\hline 2 & 3 & $\mathrm{NO}$ & $\mathrm{NO}$ & $\mathrm{NO}$ & $\mathrm{NO}$ & $\mathrm{NO}$ \\
\hline 2 & 4 & $\mathrm{NO}$ & $\mathrm{NO}$ & $\mathrm{NO}$ & $\mathrm{NO}$ & $\mathrm{NO}$ \\
\hline 3 & 1 & $\mathrm{NO}$ & $\mathrm{NO}$ & $\mathrm{NO}$ & $\mathrm{NO}$ & $\mathrm{NO}$ \\
\hline 3 & 2 & $\mathrm{NO}$ & $\mathrm{NO}$ & $\mathrm{NO}$ & $\mathrm{NO}$ & $\mathrm{NO}$ \\
\hline 3 & 3 & $\mathrm{NO}$ & $\mathrm{NO}$ & $\mathrm{NO}$ & $\mathrm{NO}$ & $\mathrm{NO}$ \\
\hline 3 & 4 & $\mathrm{NO}$ & $\mathrm{NO}$ & $\mathrm{NO}$ & $\mathrm{NO}$ & $\mathrm{OK}, \beta=1(* *)$ \\
\hline 4 & 1 & $\mathrm{NO}$ & $\mathrm{NO}$ & $\mathrm{NO}$ & $\mathrm{NO}$ & $\mathrm{NO}$ \\
\hline 4 & 2 & $\mathrm{NO}$ & $\mathrm{NO}$ & $\mathrm{NO}$ & $\mathrm{NO}$ & $\mathrm{NO}$ \\
\hline 4 & 3 & $\mathrm{NO}$ & $\mathrm{NO}$ & $\mathrm{OK}, \gamma=1(*)$ & $\mathrm{NO}$ & $\mathrm{NO}$ \\
\hline 4 & 4 & $\mathrm{NO}$ & $\mathrm{NO}$ & $\mathrm{NO}$ & $\mathrm{NO}$ & $\mathrm{NO}$ \\
\hline
\end{tabular}

$(*) K_{22}^{-1} \simeq \mathcal{V} \sqrt{\tau_{2}}, K_{11}^{-1} \simeq \mathcal{V}^{\delta} \tau_{1}^{2-3 \delta / 2}$ with $\frac{1}{\beta}+\alpha=1+\delta, \frac{1}{\beta}>\alpha$

$(* *) K_{11}^{-1} \simeq \mathcal{V} \sqrt{\tau_{1}}, K_{22}^{-1} \simeq \mathcal{V}^{\delta} \tau_{2}^{2-3 \delta / 2}$ with $\frac{1}{\gamma}+\alpha=1+\delta, \frac{1}{\gamma}>\alpha$

Therefore we realise that the positive results represent cases where all the $N_{\text {small }}$ small Kähler moduli plus a particular combination, which is the overall volume, are stabilised. It is then straightforward to see that at this stage there will be $\left(h_{1,1}(X)-N_{\text {small }}-1\right)$ flat directions. This terminates our proof of the LARGE Volume Claim.

\section{A.3 General Picture}

We shall try now to draw some conclusions from the previous LARGE Volume Claim. This can be done by noticing that it is possible to understand the topological meaning of two of the four cases for the form of the elements of the inverse Kähler matrix.

Let us focus on the Kähler modulus $\tau_{1}$. From the general expression of the inverse Kähler matrix for an arbitrary Calabi-Yau (A.20), we deduce that in this case, dropping all the coefficients

$$
K_{11}^{-1} \simeq-\mathcal{V} k_{11 i} t^{i}+\tau_{1}^{2} .
$$

Case (1) states that $K_{11}^{-1} \simeq \tau_{1}^{2}$, therefore the quantity $k_{11 i} t^{i}$ has to vanish. This is definitely true if $k_{11 i}=0, \forall i=1, \ldots, h_{1,1}(X)$, that is if the volume is linear in $t_{1}$, the 2 -cycle volume corresponding to $\tau_{1}$. This is the definition of a three-fold with a K3 fibration structure over the base $t_{1}$. Thus we realise that Calabi-Yau K3 fibrations correspond to case (1). More precisely if the three-fold is a single fibration only $K_{11}^{-1}$ will be in case (1) but not $K_{22}^{-1}$. On the contrary, double K3 fibrations will have both $K_{11}^{-1}$ and $K_{22}^{-1}$ in case (1). Thus we have proved that 
Claim $2 K_{11}^{-1} \sim \tau_{1}^{2} \Leftrightarrow \tau_{1}$ is a K3 fiber over the base $t_{1}$.

One could wonder whether this reasoning is correct being worried about possible field redefinitions since we showed that they can change the intersection numbers. However this argument is indeed correct because, as we have explained above, when one restricts himself to changes of basis which do not alter the form of the superpotential (A.25), the form of the elements of the inverse Kähler matrix do not change as the physics depends only on them and we know that it should not be modified by changes of basis. Therefore it suffices to calculate $K_{11}^{-1}$ in one frame where the geometrical interpretation is clear.

The same procedure can be followed to prove that $K_{11}^{-1} \sim \mathcal{V} \sqrt{\tau_{1}}$ if and only if $\tau_{1}$ is a blow-up mode resolving a point-like singularity. By definition, these blow-up modes are cycles such that the change in the volume of the Calabi-Yau as the cycle is collapsed only goes as the volume of the cycle, with no dependence on the overall volume.

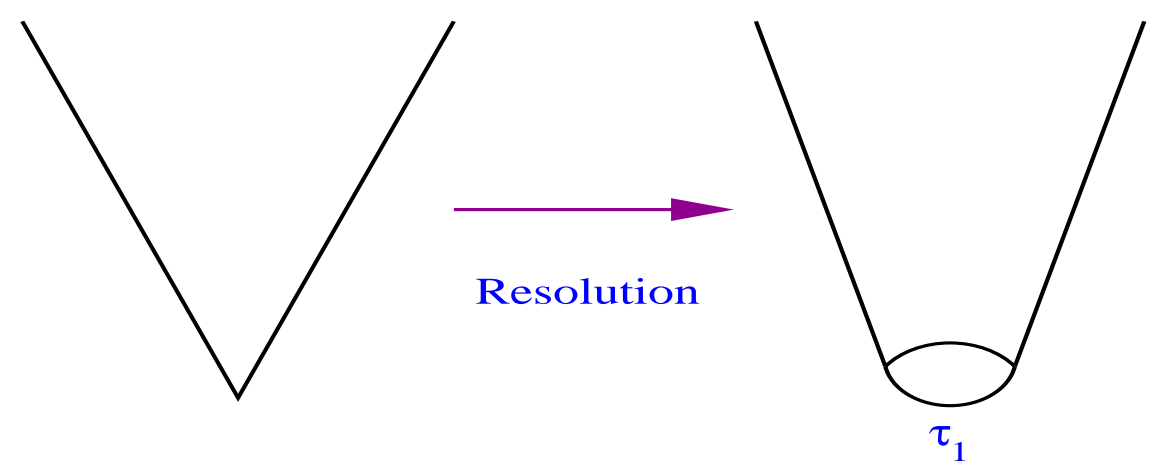

Figure 6: Blow-up cycle $\tau_{1}$ resolving a point-like singularity.

Therefore if $\tau_{1}$ is a blow-up then, in a suitable basis, we can always write the volume as

$$
\mathcal{V}=f_{3 / 2}\left(\tau_{j}\right)-\tau_{1}^{3 / 2}, \quad j \neq 1
$$

where $f_{3 / 2}\left(\tau_{j}\right)$ is an homogeneous function of degree $3 / 2$. In fact, in this case a change of $\tau_{1}, \delta \tau_{1}$, would generate a change of the total volume of the form

$$
\delta \mathcal{V}=\frac{\partial \mathcal{V}}{\partial \tau_{1}} \delta \tau_{1}=-\frac{3}{2} \sqrt{\tau_{1}} \delta \tau_{1}
$$

Let us approximate the volume A.165 as $\mathcal{V} \simeq f_{3 / 2}\left(\tau_{j}\right)$ and calculate the Kähler matrix

$$
\frac{\partial K}{\partial \tau_{1}} \simeq \frac{\sqrt{\tau_{1}}}{\mathcal{V}} \Longrightarrow\left\{\begin{array}{c}
K_{11}=\frac{\partial^{2} K}{\partial \tau_{1}^{2}} \simeq \frac{1}{\sqrt{\tau_{1}} \mathcal{V}} \\
K_{1 j}=\frac{\partial^{2} K}{\partial \tau_{j} \partial \tau_{1}} \simeq-\frac{\sqrt{\tau_{1}}}{\mathcal{V}^{2}} \frac{\partial \mathcal{V}}{\partial \tau_{j}} \simeq o\left(\frac{1}{\mathcal{V}^{5 / 3}}\right), j \neq 1
\end{array}\right.
$$

It turns out that $\frac{K_{1 j}}{K_{11}} \simeq o\left(\frac{1}{\mathcal{V}^{2 / 3}}\right) \ll 1, j \neq 1$ and so we can immediately deduce the leading order term in the "11" element of the inverse Kähler matrix by simply taking the inverse 
of $K_{11}$, which gives case (3) or more explicitly $K_{11}^{-1} \simeq \mathcal{V} \sqrt{\tau_{1}}$. But does $K_{11}^{-1} \sim \mathcal{V} \sqrt{\tau_{1}}$ imply a form of the volume as $\mathcal{V}=f_{3 / 2}\left(\tau_{j}\right)-\tau_{1}^{3 / 2}$ with $j \neq 1$ ? We shall prove now that this is indeed the case. Let us focus on $N_{\text {small }}=1$ without loss of generality. Then

$$
K_{i j} \equiv \frac{\partial^{2} K}{\partial \tau_{i} \partial \tau_{j}}=\frac{2}{\mathcal{V}}\left(\frac{1}{\mathcal{V}} \frac{\partial \mathcal{V}}{\partial \tau_{i}} \frac{\partial \mathcal{V}}{\partial \tau_{j}}-\frac{\partial^{2} \mathcal{V}}{\partial \tau_{i} \partial \tau_{j}}\right), \text { for } i, j=1,2
$$

We can then invert the Kähler matrix and find (by denoting $\frac{\partial \mathcal{V}}{\partial \tau_{j}} \equiv \mathcal{V}_{j}$ and similarly for the second derivative)

$$
K_{11}^{-1}=\frac{\mathcal{V}}{2}\left[\frac{\frac{1}{\mathcal{V}} \mathcal{V}_{2}^{2}-\mathcal{V}_{22}}{\left(\frac{1}{\mathcal{V}} \mathcal{V}_{1}^{2}-\mathcal{V}_{11}\right)\left(\frac{1}{\mathcal{V}} \mathcal{V}_{2}^{2}-\mathcal{V}_{22}\right)-\left(\frac{1}{\mathcal{V}} \mathcal{V}_{1} \mathcal{V}_{2}-\mathcal{V}_{12}\right)^{2}}\right]
$$

Now if we impose that at leading order $K_{11}^{-1}=c_{1} \mathcal{V} \sqrt{\tau_{1}}$ with $c_{1} \in \mathbb{R} \backslash\{0\}$, we get that at leading order

$$
\left[\frac{\frac{1}{\mathcal{V}} \mathcal{V}_{2}^{2}-\mathcal{V}_{22}}{\left(\frac{1}{\mathcal{V}} \mathcal{V}_{1}^{2}-\mathcal{V}_{11}\right)\left(\frac{1}{\mathcal{V}} \mathcal{V}_{2}^{2}-\mathcal{V}_{22}\right)-\left(\frac{1}{\mathcal{V}} \mathcal{V}_{1} \mathcal{V}_{2}-\mathcal{V}_{12}\right)^{2}}\right]=2 c_{1} \sqrt{\tau_{1}}
$$

Now using the homogeneity property of the volume in terms of the 4-cycle moduli, $\tau_{1} \mathcal{V}_{1}+$ $\tau_{2} \mathcal{V}_{2}=\frac{3}{2} \mathcal{V}$, we derive

$$
\mathcal{V}_{2}=\frac{3 \mathcal{V}}{2 \tau_{2}}-\frac{\mathcal{V}_{1} \tau_{1}}{\tau_{2}}, \quad \mathcal{V}_{12}=\frac{\mathcal{V}_{1}}{2 \tau_{2}}-\frac{\mathcal{V}_{11} \tau_{1}}{\tau_{2}}, \quad \mathcal{V}_{22}=\frac{3 \mathcal{V}-4 \mathcal{V}_{1} \tau_{1}+4 \mathcal{V}_{11} \tau_{1}^{2}}{4 \tau_{2}^{2}}
$$

Now plugging (A.171) back in A.170), we find that at leading order

$$
3 \mathcal{V}\left(1+2 c_{1} \sqrt{\tau_{1}} \mathcal{V}_{11}\right)-2 \mathcal{V}_{1}\left(2 \tau_{1}+c_{1} \sqrt{\tau_{1}} \mathcal{V}_{1}\right)=0
$$

We can now write the general form of $\mathcal{V}_{1}$ as $\mathcal{V}_{1}=c_{2} \mathcal{V}^{\alpha} \tau_{1}^{\frac{(1-3 \alpha)}{2}}$ with $c_{2} \in \mathbb{R} \backslash\{0\}$ and $\alpha \leq 1$. It follows then that

$$
\mathcal{V}_{11}=c_{2} \frac{\partial}{\partial \tau_{1}}\left(\mathcal{V}^{\alpha} \tau_{1}^{\frac{(1-3 \alpha)}{2}}\right)=\alpha c_{2}^{2} \mathcal{V}^{2 \alpha-1} \tau_{1}^{1-3 \alpha}+c_{2} \frac{(1-3 \alpha)}{2} \mathcal{V}^{\alpha} \tau_{1}^{-\frac{(3 \alpha+1)}{2}}
$$

which for $\alpha<1, \alpha \neq 1 / 3$, at leading order reduces to

$$
\mathcal{V}_{11}=c_{2} \frac{(1-3 \alpha)}{2} \mathcal{V}^{\alpha} \tau_{1}^{-\frac{(3 \alpha+1)}{2}}
$$

while for $\alpha=1 / 3$ reads

$$
\mathcal{V}_{11}=\frac{c_{2}^{2}}{3} \mathcal{V}^{-1 / 3}
$$

and for $\alpha=1$ becomes

$$
\mathcal{V}_{11}=c_{2} \frac{\mathcal{V}}{\tau_{1}^{2}}\left(c_{2}-1\right)
$$

Now if $1 / 2<\alpha \leq 1$, then (A.172) at leading order looks like

$$
3 \mathcal{V}_{11}=c_{2}^{2} \mathcal{V}^{2 \alpha-1} \tau_{1}^{(1-3 \alpha)}
$$


For $1 / 2<\alpha<1$, using (A.174, (A.177) gives

$$
3 c_{2} \frac{(1-3 \alpha)}{2} \mathcal{V}^{\alpha} \tau_{1}^{-\frac{(3 \alpha+1)}{2}}=c_{2}^{2} \mathcal{V}^{2 \alpha-1} \tau_{1}^{(1-3 \alpha)}
$$

which at leading order reduces to

$$
3 c_{2} \frac{(1-3 \alpha)}{2} \mathcal{V}^{\alpha} \tau_{1}^{-\frac{(3 \alpha+1)}{2}}=0,
$$

with the solution $\alpha=1 / 3$ that is in contradiction with the fact that we are considering $\alpha>1 / 2$. For $\alpha=1$, using (A.176), (A.177) becomes

$$
3\left(c_{2}-1\right)=c_{2},
$$

but the solution $c_{2}=3 / 2 \Rightarrow \tau_{1} \mathcal{V}_{1}=\frac{3}{2} \mathcal{V}$, using the homogeneity property of the volume, $\tau_{1} \mathcal{V}_{1}+\tau_{2} \mathcal{V}_{2}=\frac{3}{2} \mathcal{V}$, would imply $\mathcal{V}_{2}=0$ and so we have to reject it. On the contrary if $\alpha=1 / 2$, then (A.172) at leading order takes the form

$$
6 c_{1} \sqrt{\tau_{1}} \mathcal{V}_{11}=\left(2 c_{1} c_{2}^{2}-3\right),
$$

and by means of (A.174), this expression at leading order becomes

$$
c_{1} c_{2} \mathcal{V}^{1 / 2} \tau_{1}^{-\frac{3}{4}}=0
$$

that clearly admits no possible solution. Finally if $\alpha<1 / 2$, then (A.172) at leading order becomes

$$
3 \mathcal{V}\left(1+2 c_{1} \sqrt{\tau_{1}} \mathcal{V}_{11}\right)=0
$$

Due to (A.174), A.183 for $\alpha \neq 1 / 3$ reads

$$
3 \mathcal{V}\left(1+c_{1} c_{2}(1-3 \alpha) \mathcal{V}^{\alpha} \tau_{1}^{-\frac{3 \alpha}{2}}\right)=0,
$$

whereas using (A.175), A.183) for $\alpha=1 / 3$ takes the form

$$
0=3 \mathcal{V}\left(1+2 c_{1} \sqrt{\tau_{1}} \frac{c_{2}^{2}}{3} \mathcal{V}^{-1 / 3}\right) \simeq 3 \mathcal{V}
$$

which is impossible to solve. Now focusing on (A.184), if $\alpha<0$ we do not find any solution, whereas if $0<\alpha<1 / 2$, A.184 reduces to

$$
3 c_{1} c_{2}(1-3 \alpha) \mathcal{V}^{\alpha+1} \tau_{1}^{-\frac{3 \alpha}{2}}=0
$$

which is solved by $\alpha=1 / 3$ that is in disagreement with the fact that we are considering $\alpha \neq 1 / 3$. Lastly for $\alpha=0$, (A.184) looks like

$$
3 \mathcal{V}\left(1+c_{1} c_{2}\right)=0
$$

which admits a solution of the form $c_{2}=-\frac{1}{c_{1}}$. Therefore we have $\mathcal{V}_{1}=-\frac{\sqrt{\tau_{1}}}{c_{1}}$ and $\mathcal{V}_{11}=$ $-\frac{1}{2 c_{1} \sqrt{\tau_{1}}}$ that imply an overall volume of the form $\mathcal{V}=\lambda_{2} \tau_{2}^{3 / 2}-\lambda_{1} \tau_{1}^{3 / 2}$. It is easy now to generalise this result for $N_{\text {small }}>1$ given that we have shown that 
Claim $3 K_{11}^{-1} \sim \mathcal{V} \sqrt{\tau_{1}} \Leftrightarrow \mathcal{V}=f_{3 / 2}\left(\tau_{j}\right)-\tau_{1}^{3 / 2}$ with $j \neq 1 \Leftrightarrow \tau_{1}$ is the only blow-up mode resolving a point-like singularity.

We point out also that the stressing that the blow-up has to resolve a point-like singularity is exactly related to the fact that it has to be a purely local effect. In fact, an expression for the overall volume of the form

$$
\mathcal{V}=\tau_{b}^{3 / 2}-\tau_{b} \tau_{1}^{1 / 2}-\tau_{1}^{3 / 2}
$$

would still enable us to take a sensible large volume limit by sending $\tau_{b}$ large and keeping $\tau_{1}$ small, but this case would correspond to the resolution of a hyperplane or line-like singularity. If we approximate the volume as $\mathcal{V} \simeq \tau_{b}^{3 / 2}$, this can be seen from the fact that the change of $\mathcal{V}$ with the increase of the cycle size $\tau_{1}$ does not depend on powers of $\tau_{1}$ alone as in (A.166) but it looks like

$$
\delta \mathcal{V}=\frac{\partial \mathcal{V}}{\partial \tau_{1}} \delta \tau_{1} \simeq-\frac{1}{2} \frac{\tau_{b}}{\sqrt{\tau_{1}}} \delta \tau_{1} \simeq-\frac{1}{2} \frac{\mathcal{V}^{2 / 3}}{\sqrt{\tau_{1}}} \delta \tau_{1}
$$

Moreover the case (A.188) gives rise to an inverse Kähler metric of the form $K_{11}^{-1} \simeq \mathcal{V}^{1 / 3} \tau_{1}^{3 / 2}$ which does not satisfy the condition of the LARGE Volume Claim exactly because $\tau_{1}$ is not resolving a point-like singularity.

Let us show now that if we have $N_{\text {small }}=2$ with one small modulus $\tau_{2}$ which is a local blow-up mode then the cross term $K_{12}^{-1}$ has to be in case (1): $K_{12}^{-1} \sim \tau_{1} \tau_{2}$. Without loss of generality we can consider just one large modulus $\tau_{3}$ and so the volume will look like $\mathcal{V}=f_{3 / 2}\left(\tau_{3}, \tau_{1}\right)-\tau_{2}^{3 / 2}$. The computation of the Kähler metric gives an expression like A.168) but now for $i, j=1,3$ with in addition:

$$
K_{22}=\frac{3}{2 \mathcal{V} \sqrt{\tau_{2}}}, \quad K_{2 j}=-\frac{3 \sqrt{\tau_{2}}}{\mathcal{V}^{2}} \mathcal{V}_{j}, \quad \text { with } j=1,3,
$$

and the "12" element of the inverse Kähler metric in full generality reads

$$
K_{12}^{-1}=\frac{\tau_{2} \mathcal{V}^{2}\left(\mathcal{V}_{1} \mathcal{V}_{13}-\mathcal{V}_{1} \mathcal{V}_{33}\right)}{\left(3 \tau_{2}^{3 / 2}-\mathcal{V}\right)\left(\mathcal{V}_{11} \mathcal{V}_{3}^{2}+\mathcal{V}_{33} \mathcal{V}_{1}^{2}-2 \mathcal{V}_{1} \mathcal{V}_{3} \mathcal{V}_{13}\right)-\mathcal{V}^{2}\left(\mathcal{V}_{13}^{2}-\mathcal{V}_{11} \mathcal{V}_{33}\right)}
$$

Using again the homogeneity property of the volume, we can find the following relations

$$
\begin{gathered}
\mathcal{V}_{3}=\frac{1}{\tau_{3}}\left[\frac{3}{2}\left(\mathcal{V}+\tau_{2}^{3 / 2}\right)-\tau_{1} \mathcal{V}_{1}\right], \quad \mathcal{V}_{13}=\frac{1}{\tau_{3}}\left[\frac{\mathcal{V}_{1}}{2}-\tau_{1} \mathcal{V}_{11}\right], \\
\mathcal{V}_{33}=\frac{1}{4 \tau_{3}^{2}}\left[3\left(\mathcal{V}+\tau_{2}^{3 / 2}\right)+4 \tau_{1}\left(\tau_{1} \mathcal{V}_{11}-\mathcal{V}_{1}\right)\right],
\end{gathered}
$$

which substituted back in (A.191) give the final result

$$
K_{12}^{-1}=\frac{2 \mathcal{V}^{2} \tau_{1} \tau_{2}}{2 \mathcal{V}^{2}+6 \mathcal{V} \tau_{2}^{3 / 2}-9 \tau_{2}^{3}} \simeq \tau_{1} \tau_{2}
$$

Similarly one can check the correctness of Claim 3 by finding at leading order $K_{22}^{-1} \sim \mathcal{V} \sqrt{\tau_{2}}$. Let us summarise this result in the following 
Claim 4 If $N_{\text {small }}=2$ and $K_{22}^{-1} \sim \mathcal{V} \sqrt{\tau_{2}} \Rightarrow K_{12}^{-1} \sim \tau_{1} \tau_{2}$.

We immediately realise that this Claim rules out the possible LARGE Volume minima along the directions (II) and (III) for the case (2), (3) and (4). However following arguments similar to the ones presented to prove Claim 3, one can show that if $K_{22}^{-1} \sim \mathcal{V} \sqrt{\tau_{2}}$ and so $K_{12}^{-1} \sim \tau_{1} \tau_{2}, K_{11}^{-1}$ can never be in case (4). Hence also the new would-be LVS along the directions (II) and (III) for the case (1) have to be rejected because mathematically inconsistent.

Claim 4 also implies that the LVS along the direction (I) for case (2) and (3) is viable only if $K_{j j}^{-1} \sim \mathcal{V} h_{1 / 2}^{(j)}\left(\tau_{1}, \tau_{2}\right)$ with $\frac{\partial^{2} h^{(j)}}{\partial \tau_{1} \partial \tau_{2}} \neq 0 \forall j=1,2$. In fact if $\frac{\partial^{2} h^{(j)}}{\partial \tau_{1} \partial \tau_{2}}$ were vanishing, then Claim 4 would imply $K_{12}^{-1}$ in case (1) and not (2) or (3). In reality we understand these two cases better by realising that we can go further in our connection of the topological features of the Calabi-Yau with the elements of $K^{-1}$. In fact, one could wonder what happens when a singularity is not resolved by just one local blow-up cycle but by several independent local blow-ups.
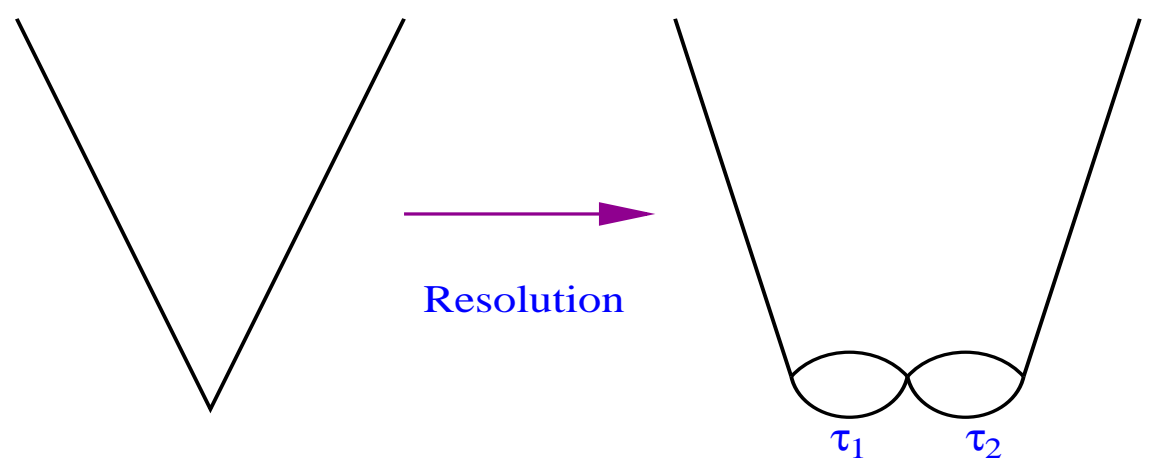

Figure 7: Resolution by two independent blow-ups $\tau_{1}$ and $\tau_{2}$.

Focusing on the case $N_{\text {small }}=2$, in a suitable basis, the overall volume will take the general form

$$
\mathcal{V}=\tau_{b}^{3 / 2}-g_{3 / 2}\left(\tau_{1}, \tau_{2}\right)
$$

with $g_{3 / 2}\left(\tau_{1}, \tau_{2}\right) \neq \tau_{1}^{3 / 2}+\tau_{2}^{3 / 2}$ since in that special case $\tau_{1}$ and $\tau_{2}$ would be blow-up cycles resolving two different point-like singularities. If the total volume is given by (A.193) then the scaling with the volume of the elements of the Kähler metric is (denoting $\partial g_{3 / 2} / \partial \tau_{j} \equiv f_{j}$ and similarly for the second derivative):

$$
\begin{aligned}
& K_{b b} \sim \frac{1}{\mathcal{V} \sqrt{\tau_{b}}}+\frac{\tau_{b}}{\mathcal{V}^{2}} \sim \frac{1}{\mathcal{V}^{4 / 3}}, \quad K_{12} \sim \frac{g_{1} g_{2}}{\mathcal{V}^{2}}+\frac{g_{12}}{\mathcal{V}} \sim \frac{1}{\mathcal{V}} \\
& K_{j b} \sim \frac{\sqrt{\tau_{b}}}{\mathcal{V}^{2}} g_{j} \sim \frac{1}{\mathcal{V}^{5 / 3}}, \quad K_{j j} \sim \frac{g_{j}^{2}}{\mathcal{V}^{2}}+\frac{g_{j j}}{\mathcal{V}} \sim \frac{1}{\mathcal{V}}, \quad j=1,2
\end{aligned}
$$


therefore producing

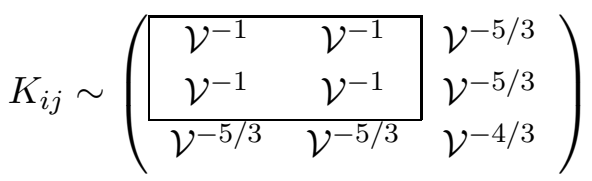

where we have highlighted with a box the submatrix with the leading powers of the volume. We have just to inverting this submatrix to get $K_{j j}^{-1}$ for $j=1,2$ and $K_{12}^{-1}$ which turn out to be given by

$$
\begin{array}{ll}
K_{11}^{-1} \sim \mathcal{V}\left(\frac{g_{22}}{2 \Delta}\right)=\mathcal{V} h_{1 / 2}^{(1)}\left(\tau_{1}, \tau_{2}\right), & K_{22}^{-1} \sim \mathcal{V}\left(\frac{g_{11}}{2 \Delta}\right)=\mathcal{V} h_{1 / 2}^{(2)}\left(\tau_{1}, \tau_{2}\right), \\
K_{12}^{-1} \sim \mathcal{V}\left(\frac{g_{12}}{2 \Delta}\right)=\mathcal{V} f_{1 / 2}\left(\tau_{1}, \tau_{2}\right), & \text { where } \Delta \equiv g_{11} g_{22}-g_{12}^{2} .
\end{array}
$$

Following arguments similar to the ones used to prove Claim 3 we can also show that starting from $K_{11}^{-1} \sim \mathcal{V} h_{1 / 2}^{(1)}\left(\tau_{1}, \tau_{2}\right)$ with $h^{(1)}$ really dependent on both the small moduli, the form of the volume has to be (A.193). A good intuition for this result is that by setting $\tau_{1}=\tau_{2}$, this is the only way to recover Claim 3 . Therefore we have shown that

Claim $5 K_{11}^{-1} \sim \mathcal{V} h_{1 / 2}^{(1)}\left(\tau_{1}, \tau_{2}\right)$ and $K_{22}^{-1} \sim \mathcal{V} h_{1 / 2}^{(2)}\left(\tau_{1}, \tau_{2}\right) \Leftrightarrow \mathcal{V}=f_{3 / 2}\left(\tau_{j}\right)-g_{3 / 2}\left(\tau_{1}, \tau_{2}\right)$ with $j \neq 1,2 \Leftrightarrow \tau_{1}$ and $\tau_{2}$ are two independent blow-up modes resolving the same point-like singularity,

along with

Claim 6 If $K_{11}^{-1} \sim \mathcal{V} h_{1 / 2}^{(1)}\left(\tau_{1}, \tau_{2}\right)$ and $K_{22}^{-1} \sim \mathcal{V} h_{1 / 2}^{(2)}\left(\tau_{1}, \tau_{2}\right) \Rightarrow K_{12}^{-1} \sim \mathcal{V} f_{1 / 2}\left(\tau_{1}, \tau_{2}\right)$.

In generality we can conclude that whenever $K_{j j}^{-1} \simeq \mathcal{V} h_{1 / 2}^{(j)}\left(\tau_{1}, \tau_{2}, \ldots, \tau_{N_{\text {small }}}\right)$ then $\tau_{j}$ is a blow-up resolving a point-like singularity. Moreover, if $h_{1 / 2}^{(j)}$ depends only on $\tau_{j}$, then $\tau_{j}$ will be the only blow-up cycle resolving the singularity and $K_{i j}^{-1} \sim \tau_{i} \tau_{j} \forall i \neq j=1, \ldots, N_{\text {small }}$; on the contrary, if $h_{1 / 2}^{(j)}$ depends on several 4-cycle moduli, say $\tau_{j}$ and $\tau_{k}$ for $j \neq k$, the singularity is resolved by all those independent 4-cycles with $K_{j k}^{-1} \sim \mathcal{V} f_{1 / 2}\left(\tau_{j}, \tau_{k}\right)$ and $K_{i l}^{-1} \sim \tau_{i} \tau_{j} \forall i \neq l=1, \ldots, N_{\text {small }}$ for $l=j, k$.

These considerations imply that the would-be LVS along direction (I) for case (2) is mathematically inconsistent. Thus for $N_{\text {small }}=2$ we are left with just two cases that give rise to a LARGE Volume minimum located at $\mathcal{V} \sim e^{a_{1} \tau_{1}} \sim e^{a_{2} \tau_{2}}$ :

1. $K_{12}^{-1} \sim \tau_{1} \tau_{2}, K_{j j}^{-1} \sim \mathcal{V} \sqrt{\tau_{j}} \forall j=1,2$ where $\tau_{1}$ and $\tau_{2}$ are local blow-up modes resolving two different point-like singularities;

2. $K_{12}^{-1} \sim \mathcal{V} f_{1 / 2}\left(\tau_{1}, \tau_{2}\right), K_{j j}^{-1} \sim \mathcal{V} h_{1 / 2}^{(j)}\left(\tau_{1}, \tau_{2}\right) \forall j=1,2$ where $\tau_{1}$ and $\tau_{2}$ are two independent blow-up modes resolving the same point-like singularity.

The only difference between these two cases is that the first one works always whereas the second one gives a LVS only if, writing the volume as $\mathcal{V}=f_{3 / 2}\left(\tau_{j}\right)-g_{3 / 2}\left(\tau_{1}, \tau_{2}\right)$ with $j \neq 1,2$, the homogeneous function $g_{3 / 2}$ is symmetric in $\tau_{1}$ and $\tau_{2}$. This can be seen easily by comparing the solution of the two minimisation equations $\frac{\partial V}{\partial \tau_{1}}=0$ and $\frac{\partial V}{\partial \tau_{2}}=0$, then substituting the solution we are looking for, that is $a_{1} \tau_{1} \simeq a_{2} \tau_{2}$, recalling (A.195) and lastly finding that we do not get a contradiction only if $g_{3 / 2}$ is symmetric. 


\section{References}

[1] S. B. Giddings, S. Kachru and J. Polchinski, "Hierarchies from fluxes in string compactifications," Phys. Rev. D 66 (2002) 106006 [arXiv:hep-th/0105097].

[2] M. R. Douglas and S. Kachru, "Flux compactification," Rev. Mod. Phys. 79 (2007) 733 [arXiv:hep-th/0610102]; F. Denef, M. R. Douglas and S. Kachru, "Physics of string flux compactifications," arXiv:hep-th/0701050.

[3] R. Blumenhagen, B. Kors, D. Lust and S. Stieberger, "Four-dimensional String Compactifications with D-Branes, Orientifolds and Fluxes," Phys. Rept. 445 (2007) 1 [arXiv:hep-th/0610327].

[4] E. Witten, "Non-Perturbative Superpotentials in String Theory", Nucl. Phys. B 474 (1996) 343 [arXiv:hep-th/9604030].

[5] S. Kachru, R. Kallosh, A. Linde and S. P. Trivedi, "De Sitter vacua in string theory," Phys. Rev. D 68 (2003) 046005 [arXiv:hep-th/0301240].

[6] K. Becker, M. Becker, M. Haack and J. Louis, "Supersymmetry breaking and alpha'-corrections to flux induced potentials," JHEP 0206 (2002) 060 [arXiv:hep-th/0204254].

[7] V. Balasubramanian and P. Berglund, "Stringy corrections to Kaehler potentials, SUSY breaking, and the cosmological constant problem," JHEP 0411 (2004) 085 [arXiv:hep-th/0408054].

[8] V. Balasubramanian, P. Berglund, J. P. Conlon and F. Quevedo, "Systematics of moduli stabilisation in Calabi-Yau flux compactifications," JHEP 0503 (2005) 007 [arXiv:hep-th/0502058];

[9] J. P. Conlon, F. Quevedo and K. Suruliz, "Large-volume flux compactifications: Moduli spectrum and D3/D7 soft supersymmetry breaking," JHEP 0508 (2005) 007 [arXiv:hep-th/0505076].

[10] M. Berg, M. Haack and B. Kors, "String loop corrections to Kaehler potentials in orientifolds," JHEP 0511 (2005) 030 [arXiv:hep-th/0508043]. M. Berg, M. Haack and B. Kors, "On volume stabilization by quantum corrections," Phys. Rev. Lett. 96 (2006) 021601 [arXiv:hep-th/0508171].

[11] G. von Gersdorff and A. Hebecker, "Kaehler corrections for the volume modulus of flux compactifications," Phys. Lett. B 624 (2005) 270 [arXiv:hep-th/0507131].

[12] M. Cicoli, J. P. Conlon and F. Quevedo, "Systematics of String Loop Corrections in Type IIB Calabi-Yau Flux Compactifications," arXiv:0708.1873 [hep-th].

[13] M. Berg, M. Haack and E. Pajer, "Jumping Through Loops: On Soft Terms from Large Volume Compactifications," arXiv:0704.0737 [hep-th].

[14] R. Blumenhagen, S. Moster and E. Plauschinn, "Moduli Stabilisation versus Chirality for MSSM like Type IIB Orientifolds", arXiv:0711.3389 [hep-th].

[15] L. Covi, M. Gomez-Reino, C. Gross, J. Louis, G. A. Palma and C. A. Scrucca, "de Sitter vacua in no-scale supergravities and Calabi-Yau string models," arXiv:0804.1073 [hep-th].

[16] E. Palti, G. Tasinato and J. Ward, "WEAKLY-coupled IIA Flux Compactifications," arXiv:0804.1248 [hep-th]. 
[17] N. Arkani-Hamed, S. Dimopoulos and G. R. Dvali, "The hierarchy problem and new dimensions at a millimeter", Phys. Lett. B 429 (1998) 263 [arXiv:hep-ph/9803315]; I. Antoniadis, N. Arkani-Hamed, S. Dimopoulos and G. R. Dvali, "New dimensions at a millimeter to a Fermi and superstrings at a TeV", Phys. Lett. B 436 (1998) 257 [arXiv:hep-ph/9804398].

[18] Y. Aghababaie, C. P. Burgess, S. L. Parameswaran and F. Quevedo, "Towards a naturally small cosmological constant from branes in 6D supergravity," Nucl. Phys. B 680 (2004) 389 [arXiv:hep-th/0304256]; C. P. Burgess, "Towards a natural theory of dark energy: Supersymmetric large extra dimensions," AIP Conf. Proc. 743 (2005) 417 [arXiv:hep-th/0411140].

[19] J. P. Conlon, "The QCD axion and moduli stabilisation," JHEP 0605 (2006) 078 [arXiv:hep-th/0602233].

[20] F. Denef, M. R. Douglas and B. Florea, "Building a Better Racetrack", JHEP 0460 (2004) 034 [arXiv:hep-th/0404257].

[21] V. Kaplunovsky and J. Louis, "Field dependent gauge couplings in locally supersymmetric effective quantum field theories," Nucl. Phys. B 422 (1994) 57 [arXiv:hep-th/9402005].

[22] S. R. Coleman and E. Weinberg, "Radiative Corrections As The Origin Of Spontaneous Symmetry Breaking," Phys. Rev. D 7 (1973) 1888.

[23] S. Ferrara, C. Kounnas and F. Zwirner, "Mass formulae and natural hierarchy in string effective supergravities," Nucl. Phys. B 429 (1994) 589 [Erratum-ibid. B 433 (1995) 255] [arXiv:hep-th/9405188].

[24] J. P. Conlon and F. Quevedo, "Astrophysical and Cosmological Implications of Large Volume String Compactifications", JCAP 0708 (2007) 019 [arXiv:hep-th/0705.3460].

[25] K. Bobkov, "Volume stabilization via alpha' corrections in type IIB theory with fluxes," JHEP 0505 (2005) 010 [arXiv:hep-th/0412239].

[26] J. P. Conlon, S. S. Abdussalam, F. Quevedo and K. Suruliz, "Soft SUSY breaking terms for chiral matter in IIB string compactifications," JHEP 0701 (2007) 032 [arXiv:hep-th/0610129].

[27] J. P. Conlon, C. H. Kom, K. Suruliz, B. C. Allanach and F. Quevedo, "Sparticle Spectra and LHC Signatures for Large Volume String Compactifications," JHEP 0708 (2007) 061 [arXiv:0704.3403 [hep-ph]].

[28] J. P. Conlon, "Mirror Mediation," arXiv:0710.0873 [hep-th].

[29] J. P. Conlon and F. Quevedo, "Kaehler moduli inflation," JHEP 0601 (2006) 146 [arXiv:hep-th/0509012];

[30] J. R. Bond, L. Kofman, S. Prokushkin and P. M. Vaudrevange, "Roulette inflation with Kaehler moduli and their axions," Phys. Rev. D 75 (2007) 123511 [arXiv:hep-th/0612197].

[31] H. X. Yang and H. L. Ma, "Two-field Kähler moduli inflation on large volume moduli stabilization," arXiv:0804.3653 [hep-th].

[32] A. Misra and P. Sukhla, "Large Volume Axionic Swiss-Cheese Inflation," arXiv:0712.1260 [hep-th]. 